\title{
Sistemas de reação-difusão com acoplamento na fronteira e a propriedade de Morse-Smale
}

\section{Rita de Cássia D. Sodré Broche}

\author{
Tese apresentada \\ ao \\ Instituto de Matemática e Estatística \\ da \\ Univiersidade de São Paulo \\ para \\ obtenção do grau \\ de \\ Doutor em Matemática \\ Área de Concentração: Matemática
}

Orientador: Prof. Dr. Luiz Augusto Fernandes de Oliveira

São Paulo

Maio de 2005 


\section{SISTEMAS DE REAÇÃO-DIFUSÃO COM ACOPLAMENTO NA FRONTEIRA E A PROPRIEDADE DE MORSE-SMALE}

Rita de Cássia Dornelas Sodré Broche

Orientador: Prof. Dr. Luiz Augusto Fernandes de Oliveira

Tese apresentada ao Instituto de Matemática

e Estatística da Universidade de São Paulo, como requisito parcial à obtenção do título de Doutor em Ciências. Área: Matemática.

São Paulo

Maio de 2005 
Aos meus pais, Laerte e Conceição, meu esposo Osnel e meu filho Juan 


\section{Agradecimentos}

A Deus por estar presente em todos os momentos da minha vida.

À Pós-Graduação do Instituto de Matemática e Estatística - USP - por me receber no curso de doutorado e à CAPES pela ajuda financeira.

Aos professores que, de uma forma ou outra, contribuiram para a minha formação, em especial, meu orientador, Prof. Dr. Luiz Augusto F. de Oliveira, pela paciência, dedicação, disponibilidade e confiança, e os professores, Dr. Antônio Luiz Pereira (IMEUSP) e Dr. Alexandre Nolasco de Carvalho (IMECC-USP-São Carlos), pelas valiosas sugestões e discussões que muito contribuiram para o desenvolvimento deste trabalho.

Aos funcionários da Secretaria de Pós-Graduação e da Biblioteca, pela simpatia, atenção e dedicação para comigo.

À minha família, pelo carinho e incentivo, em especial, meus pais, Laerte e Conceição, meu esposo Osnel e meu filho Juan; todos os agradecimentos seriam pouco.

À minha amiga Luciana e meu cunhado Elier, por toda ajuda, incentivo e momentos compartilhados.

Aos colegas Carlos, Maria do Carmo, Marcone e Andréia, pelas discussões matemáticas que muito me ajudaram e também, aos colegas da sala de doutorado B-141, pelos momentos agradáveis vivenciados.

A todos vocês, MUITO OBRIGADA ! 


\section{Resumo}

Neste trabalho, estudamos sistemas de reação-difusão, em domínios unidimensionais, com acoplamento na fronteira. Tais sistemas modelam problemas de distribuição de temperatura para a junção de duas barras, de mesmo comprimento, com coeficientes de difusão distintos.

Provamos a transversalidade das variedades estável e instável de pontos de equilíbrio hiperbólicos. Para isto, reescrevemos tal sistema como uma equação com coeficiente de difusão descontínuo e estudamos a propriedade do decrescimento do número de zeros das soluções de uma equação linearizada não autônoma, assim como, a propriedade de Sturm-Liouville das soluções de um problema elíptico linear.

Apresentamos alguns resultados parciais sobre a bifurcação de pontos de equilíbrio da solução trivial, para o caso de certas não linearidades e para barras homogêneas. 


\section{Abstract}

We study one-dimensional nonlinear reaction-diffusion systems coupled through the boundary. Such systems arise as models of temperature distribution for two bars with the same lenght, joined together, with different diffusion coefficients.

We prove the transversality property of unstable and stable manifolds assuming all equilibrium points are hyperbolic. To attack this problem, we write the system as an equation with noncontinuous diffusion coefficient. We then study the property of the nonincreasing the number of zeroes of a linearized nonautonomous equation as well as the Sturm-Liouville properties of the solutions a linear elliptic problem.

We also present some partial results regarding the bifurcation of equilibrium points from the trivial solution for the case of certain nonlinearities and for homogeneous bars. 


\section{Introdução}

Sejam $a$ e $b$ funções reais positivas de classe $C^{1}$ definidas no intervalo $[0,1]$ e consideremos o seguinte sistema de equações parabólicas

$$
\left\{\begin{array}{l}
u_{t}=\left(a(x) u_{x}\right)_{x}+f(u), \quad x \in(0,1), \quad t>0 \\
v_{t}=\left(b(x) v_{x}\right)_{x}+g(v), x \in(0,1), \quad t>0
\end{array}\right.
$$

acopladas em $x=0$ pelas condições

$$
\left\{\begin{array}{l}
u(0, t)=v(0, t) \\
a(0) u_{x}(0, t)+b(0) v_{x}(0, t)=0
\end{array}\right.
$$

e em $x=1$ assumimos

$$
u(1, t)=v(1, t)=0 .
$$

Suponhamos que as funções $f$ e $g$ pertencem a $C^{2}(\mathbb{R}, \mathbb{R})$ e satisfazem

(H1) $f(0)=g(0)=0$ e $f^{\prime}(0)=g^{\prime}(0)=1$;

(H2) $\limsup _{|u| \rightarrow+\infty} \frac{f(u)}{u} \leq 0 \quad$ e $\limsup _{|v| \rightarrow+\infty} \frac{g(v)}{v} \leq 0$.

Para $(u(x, t), v(x, t)), x \in[0,1]$ e $t \geq 0$, solução do problema (0.1)-(0.3), podemos considerar a seguinte função $w$ definida em $[-1,1] \times[0,+\infty)$ por

$$
w(x, t)=\left\{\begin{array}{l}
u(-x, t), \quad x \in[-1,0], t \geq 0 \\
v(x, t), \quad x \in[0,1], t \geq 0
\end{array}\right.
$$

É fácil ver que $w$ é solução do problema parabólico unidimensional

$$
\left\{\begin{array}{l}
w_{t}=\left(a(-x) w_{x}\right)_{x}+f(w), x \in(-1,0), t>0 \\
w_{t}=\left(b(x) w_{x}\right)_{x}+g(w), x \in(0,1), t>0 \\
w(0-, t)=w(0+, t) \\
a(0) w_{x}(0-, t)=b(0) w_{x}(0+, t) \\
w(-1, t)=w(1, t)=0
\end{array}\right.
$$

onde $w(0 \pm, t)$ e $w_{x}(0 \pm, t)$ denotam os limites laterais das funções $w(\cdot, t)$ e $w_{x}(\cdot, t)$ em $x=0$, respectivamente. Este problema modela a junção de duas barras não - homogêneas de mesmo comprimento e com coeficientes de difusão distintos. 
Se $a(0)=b(0), a^{\prime}(0)=-b^{\prime}(0)$ e $f=g$, então (0.4) é equivalente ao problema

$$
\left\{\begin{array}{l}
w_{t}=\left(g(x) w_{x}\right)_{x}+f(w), x \in(-1,1), t>0 \\
w(-1, t)=w(1, t)=0
\end{array}\right.
$$

onde $g(x)=\left\{\begin{array}{l}a(-x), x \in(-1,0) \\ b(x), x \in(0,1)\end{array}\right.$.

O objetivo principal deste trabalho é demonstrar que as variedades estável e instável dos pontos de equilíbrio hiperbólicos do problema (0.1)-(0.3) se interseptam transversalmente.

Henry demonstrou em [12] que a transversalidade das variedades estável e instável é válida para uma ampla classe de equações parabólicas unidimensionais, em particular, para as equações da forma

$$
\left\{\begin{array}{l}
u_{t}=a(x) u_{x x}+f\left(x, u, u_{x}\right), x \in(0,1), t>0 \\
u(0, t)=u(1, t)=0,
\end{array}\right.
$$

onde $a$ e $f$ são funções suaves e $a(x)>0$, para todo $x \in[0,1]$.

Angenent em [1] também obteve o mesmo resultado, porém com a não linearidade não dependendo de $u_{x}$.

Carbone [4] provou a transversalidade para problemas parabólicos unidimensionais, quando o coeficiente de difusão se torna muito grande em algumas partes do domínio.

A demonstração da transversalidade, nesses trabalhos, se baseia em três pontos básicos. O primeiro deles consiste na propriedade de Sturm-Liouville, válida para o operador linearizado unidimensional, com a qual se obtém resultados sobre a simplicidade dos autovalores e sobre o número de zeros das respectivas autofunções. O segundo ponto são resultados sobre o comportamento assintótico das soluções de equações lineares, que são válidos para uma grande variedade de problemas de evolução. O terceiro e último ponto é uma propriedade das soluções de equações parabólicas lineares unidimensionais, demonstrada por Matano [14]; dessa propriedade concluímos que se $u(x, t)$ é uma solução de uma equação parabólica linear unidimensional, então o número de zeros de $u(\cdot, t)$ não pode crescer com o tempo.

O fato de podermos reescrever o problema (0.1)-(0.3) como um problema unidimensional é essencial para utilizarmos as idéias de Henry [12]. 
A organização deste trabalho é a seguinte. No Capítulo 1 provamos a existência de soluções, globalmente definidas, e a existência do atrator global. Em seguida, na Seção 1.2, demonstramos que o problema (0.4), escrito na forma unidimensional, tem a propriedade de Sturm-Liouville. Na Seção 1.3, utilizamos os resultados de [12] para estudar o comportamento assintótico das soluções das equações lineares não - autônomas.

No Capítulo 2, estudamos o número de zeros, no intervalo $(-1,1)$, das soluções de equações lineares não-autônomas relacionadas ao problema (0.4). A irregularidade das soluções nos pontos $(x, t)=(0, t)$ nos impede de usar o princípio do máximo na forma apresentada em [16] e o argumento desenvolvido por Matano em [14] (ver Lema 2.6). Por essa razão, utilizamos um método geral de aproximações de soluções anteriormente usado pelos Profs. A. Carvalho e S. Piskarev em [5] para contornar as dificuldades encontradas. A base do argumento é aproximarmos, em certo sentido, as soluções do problema linearizado irregular, relacionado ao problema (0.4), por soluções de problemas parabólicos regulares e, para estas, utilizar os resultados já existentes. Com essa aproximação, conseguimos estabelecer um resultado sobre o número de zeros das soluções de equações lineares, que nos permite demonstrar, juntamente com os resultados do Capítulo 1, a transversalidade das variedades.

No Capítulo 3, depois de caracterizarmos os espaços tangentes às variedades estável e instável dos pontos de equilíbrio hiperbólicos, demonstramos finalmente a transversalidade dessas.

Com o objetivo de descrever melhor a dinâmica de problemas do tipo (0.1)-(0.3) fazemos, no Capítulo 4, um breve estudo dos pontos de equilíbrio não-triviais para um problema particular. Consideramos o caso em que os coeficientes de difusão são funções constantes, digamos $a(x)=a>0, b(x)=b>0$ e as não linearidades são dadas por $f(u)=u-c u^{3}$ e $g(v)=v-d v^{3}$, onde $c$ e $d$ são constantes positivas.

No capítulo final apresentamos algumas generalizações dos tópicos desenvolvidos nesse trabalho e possíveis problemas para investigação futura. 


\section{Sumário}

Introdução $\quad$ xi

1 Resultados Preliminares 1

1.1 Teoremas de existência . . . . . . . . . . . . . . . 1

1.2 Propriedade de Sturm-Liouville . . . . . . . . . . . . . . . 10

1.3 Comportamento assintótico das soluções de equações lineares . . . . . . . 17

2 Estudo do número de zeros de soluções de equações lineares 33

2.1 Problema aproximado . . . . . . . . . . . . . . 35

2.2 Convergência espectral, estimativas para o resolvente e convergência do

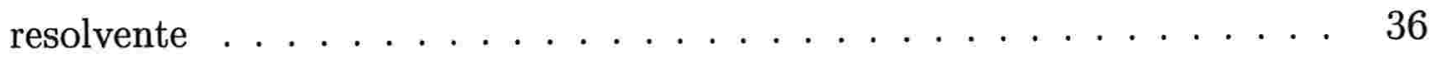

2.3 Convergência do problema aproximado . . . . . . . . . . 51

3 Transversalidade das Variedades Estável e Instável 55

4 Bifurcação de pontos de equilíbrio para um problema particular $\quad 65$

5 Conclusões e Observações $\quad 81$

$\begin{array}{ll}\text { Bibliografia } & 83\end{array}$ 


\section{Capítulo 1}

\section{Resultados Preliminares}

\subsection{Teoremas de existência}

Reescrevemos o problema parabólico (0.1)-(0.3) como uma equação de evolução. Para isto, considere o seguinte subespaço vetorial de $X=L^{2}(0,1) \times L^{2}(0,1)$,

$$
H=\left\{(u, v) \in H^{1}(0,1) \times H^{1}(0,1): u(0)=v(0), u(1)=v(1)=0\right\}
$$

munido com o produto interno

$$
\langle(u, v),(w, z)\rangle_{H}=\int_{0}^{1}\left[u^{\prime} w^{\prime}+v^{\prime} z^{\prime}\right] d x=\left\langle\left(u^{\prime}, v^{\prime}\right),\left(w^{\prime}, z^{\prime}\right)\right\rangle_{X}
$$

onde $\langle., .\rangle_{X}$ denota o produto interno canônico em $X$. É fácil ver que $H$ é um espaço de Hilbert com o produto interno definido acima.

Para todo $(u, v) \in H$, temos $u, v \in H^{1}(0,1)$ e $u(1)=v(1)=0$; assim vale a desigualdade de Poincaré para $u$ e $v$, isto é,

$$
\|u\|_{L^{2}(0,1)} \leq\left\|u^{\prime}\right\|_{L^{2}(0,1)} \text { e }\|v\|_{L^{2}(0,1)} \leq\left\|v^{\prime}\right\|_{L^{2}(0,1)} .
$$

Logo, a norma associada ao produto interno de $H$ é equivalente à norma canônica de $H^{1}(0,1) \times H^{1}(0,1)$. Dessa forma, é fácil ver que a imersão de $H$ em $X$ é contínua e compacta. Por outro lado, $H$ é denso em $X$ pois $C_{c}^{\infty}(0,1) \times C_{c}^{\infty}(0,1) \subset H$ e $C_{c}^{\infty}(0,1) \times$ $C_{c}^{\infty}(0,1)$ é denso em $X$.

Agora, seja $\tau: H \times H \longrightarrow \mathbb{R}$ a forma bilinear simétrica definida por

$$
\tau((u, v),(w, z))=\int_{0}^{1}\left[a(x) u^{\prime} w^{\prime}+b(x) v^{\prime} z^{\prime}\right] d x .
$$


Mostremos que $\tau$ é contínua e coerciva. De fato, segue da desigualdade de CauchySchwarz que

$$
\begin{aligned}
|\tau((u, v),(w, z))| & \leq \max _{x \in[0,1]}\{a(x), b(x)\}\left|\int_{0}^{1}\left(u^{\prime} w^{\prime}+v^{\prime} z^{\prime}\right) d x\right| \\
& \leq k_{1}\|(u, v)\|_{H}\|(w, z)\|_{H}
\end{aligned}
$$

para todos $(u, v),(w, z) \in H$, o que implica que $\tau$ é contínua. De forma análoga, $\tau$ é coerciva pois

$$
\begin{aligned}
\tau((u, v),(u, v)) & \geq \min _{x \in[0,1]}\{a(x), b(x)\} \int_{0}^{1}\left[\left(u^{\prime}\right)^{2}+\left(v^{\prime}\right)^{2}\right] d x \\
& =k_{2}\|(u, v)\|_{H}^{2}
\end{aligned}
$$

para todo $(u, v) \in H$, sendo $k_{2}=\min _{x \in[0,1]}\{a(x), b(x)\}>0$.

Considere o operador linear $A \in \mathcal{L}\left(H, H^{\prime}\right)$ induzido por $\tau$, isto é,

$$
\langle A(u, v),(w, z)\rangle_{H^{\prime} \times H}=\tau((u, v),(w, z))
$$

para todos $(u, v),(w, z) \in H$. Identificando $X$ com seu espaço dual $X^{\prime}$, podemos considerar $A$ como um operador não limitado fechado positivo autoadjunto $A: D(A) \subset$ $X \rightarrow X$ definido por

$$
A(u, v)(x)=-\left(\left(a(x) u_{x}\right)_{x},\left(b(x) v_{x}\right)_{x}\right), x \in(0,1)
$$

para qualquer $(u, v) \in D(A)$ sendo

$D(A)=\left\{(u, v) \in H^{2}(0,1)^{2}: u(0)=v(0), a(0) u_{x}(0)+b(0) v_{x}(0)=0, u(1)=v(1)=0\right\}$

Dessa forma, $A$ é um operador setorial em $X$ e, portanto, suas potências fracionárias estão bem definidas. Em particular, segue de [20] que $X^{0}=X, X^{1}=D(A)$ e $X^{1 / 2}=H$.

Consideremos a seguir o operador de Nemitsky associado a $f$ e $g$, isto é,

$$
N: H \longrightarrow X, N(u, v)(x)=(f(u(x)), g(v(x))), \quad x \in(0,1) .
$$

Assim, o problema (0.1)-(0.3) equivale à seguinte equação de evolução em $X$

$$
w_{t}=-A w+N(w), \quad t>0
$$

onde $w=(u, v)$. 
Lema 1.1 Se $f$ e $g \in C^{2}(\mathbb{R}, \mathbb{R})$ então a aplicação $N: H=X^{1 / 2} \longrightarrow X$ satisfaz:

(i) $N$ é localmente de classe $C^{1,1}$, isto é, $N$ é de classe $C^{1}$ e $N^{\prime}$ é localmente Lipschitz;

(ii) $N$ é compacta; de fato, $N: H \rightarrow H^{1}(0,1)^{2}$.

Demonstração. Primeiramente lembramos que se $(u, v) \in H$, então $u, v \in\{w \in$ $\left.H^{1}(0,1): w(1)=0\right\}$ onde é válido

$$
|w(x)|=\left|\int_{x}^{1} w^{\prime}(s) d s\right| \leq\left(\int_{0}^{1}\left|w^{\prime}(s)\right|^{2} d s\right)^{1 / 2}=\left\|w^{\prime}\right\|_{L^{2}(0,1)},
$$

para todo $x \in[0,1]$. Assim, se $(u, v),(w, z) \in H$ são tais que $\|(u, v)\|_{H},\|(w, z)\|_{H} \leq r$ temos, pela observação anterior, que $|u(x)| \leq\left\|u^{\prime}\right\|_{L^{2}} \leq\|(u, v)\|_{H} \leq r$, para todo $x \in$ $[0,1]$, sendo que o mesmo é válido para as funções $v, w$ e $z$.

Como $f$ e $g$ são de classe $C^{2}$ podemos considerar as seguintes constantes $k_{3}=k_{3}(r)=\sup ^{2}\left\{\left|f^{\prime}(s)\right|:|s| \leq r\right\}$ e $k_{4}=k_{4}(r)=\sup ^{2}\left\{\left|g^{\prime}(s)\right|:|s| \leq r\right\}$, sendo $r>0$. Dessa forma,

$$
\begin{aligned}
\|N(u, v)-N(w, z)\|_{X}^{2} & =\|(f(u), g(v))-(f(w), g(z))\|_{X}^{2} \\
& =\int_{0}^{1}\left[|f(u(x))-f(w(x))|^{2}+|g(v(x))-g(z(x))|^{2}\right] d x \\
& =\int_{0}^{1}\left[\left|\int_{w(x)}^{u(x)} f^{\prime}(s) d s\right|^{2}+\left|\int_{z(x)}^{v(x)} g^{\prime}(s) d s\right|^{2}\right] d x \\
& \leq \int_{0}^{1}\left[k_{3}|u(x)-w(x)|^{2}+k_{4}|v(x)-z(x)|^{2}\right] d x \\
& \leq k(r)\|(u, v)-(w, z)\|_{X}^{2} \\
& \leq k(r)\|(u, v)-(w, z)\|_{H}^{2} .
\end{aligned}
$$

Logo, $N$ é Lipschitz em conjuntos limitados. Por outro lado, não é difícil provar que a derivada de Frechet de $N$ é dada por

$$
\left[N^{\prime}\left(u_{0}, v_{0}\right)\right](u, v)=\left(f^{\prime}\left(u_{0}\right) u, g^{\prime}\left(v_{0}\right) v\right) \in X
$$

para todo $(u, v) \in H$.

Provemos a seguir que $N^{\prime}$ é Lipschitz em conjuntos limitados, o que implica $N$ ser de classe $C^{1,1}$. De fato, sejam $\left(u_{0}, v_{0}\right),\left(u_{1}, v_{1}\right) \in H$ tais que $\left\|\left(u_{0}, v_{0}\right)\right\|_{H},\left\|\left(u_{1}, v_{1}\right)\right\|_{H} \leq r$. De maneira análoga à primeira parte da demonstração, consideremos definidas as seguintes 
constantes: $k_{5}=k_{5}(r)=\sup ^{2}\left\{\left|f^{\prime \prime}(s)\right|:|s| \leq r\right\}$ e $k_{6}=k_{6}(r)=\sup ^{2}\left\{\left|g^{\prime \prime}(s)\right|:|s| \leq r\right\}$. Então, para todo $(u, v) \in H$ temos

$$
\begin{aligned}
&\left\|\left[N^{\prime}\left(u_{0}, v_{0}\right)-N^{\prime}\left(u_{1}, v_{1}\right)\right](u, v)\right\|_{X}^{2}= \int_{0}^{1}\left[\left|f^{\prime}\left(u_{0}(x)\right)-f^{\prime}\left(u_{1}(x)\right)\right|^{2}|u(x)|^{2}+\right. \\
&\left.+\left|g^{\prime}\left(v_{0}(x)\right)-g^{\prime}\left(v_{1}(x)\right)\right|^{2}|v(x)|^{2}\right] d x \\
&= \int_{0}^{1}\left[\left|\int_{u_{1}(x)}^{u_{0}(x)} f^{\prime \prime}(s) d s\right|^{2}|u(x)|^{2}+\right. \\
&\left.+\left|\int_{v_{1}(x)}^{v_{0}(x)} g^{\prime \prime}(s) d s\right|^{2}|v(x)|^{2}\right] d x \\
& \leq \int_{0}^{1}\left[k_{5}\left|u_{0}(x)-u_{1}(x)\right|^{2}|u(x)|^{2}+k_{6}\left|v_{0}(x)-v_{1}(x)\right|^{2}|v(x)|^{2}\right] d x \\
& \leq \bar{k}(r)^{2}\left[\left\|\left(u_{0}^{\prime}-u_{1}^{\prime}\right)\right\|_{L^{2}}^{2}\left\|u^{\prime}\right\|_{L^{2}}^{2}+\right.\left.\left\|\left(v_{0}^{\prime}-v_{1}^{\prime}\right)\right\|_{L^{2}}^{2}\left\|v^{\prime}\right\|_{L^{2}}^{2}\right] \\
& \leq \bar{k}(r)^{2}\left\|\left(u_{0}-u_{1}, v_{0}-v_{1}\right)\right\|_{H}^{2}\|(u, v)\|_{H}^{2},
\end{aligned}
$$

o que implica

$$
\left\|N^{\prime}\left(u_{0}, v_{0}\right)-N^{\prime}\left(u_{1}, v_{1}\right)\right\|_{\mathcal{L}(H, X)} \leq \bar{k}(r)\left\|\left(u_{0}, v_{0}\right)-\left(u_{1}, v_{1}\right)\right\|_{H}
$$

e assim concluímos a demonstração de (i). Para mostrarmos (ii), observemos que $N(u, v) \in H^{1}(0,1)^{2}$, para todo $(u, v) \in H$. De fato, se $(u, v) \in H, u$ e $v$ são absolutamente contínuas em $[0,1]$ e como $f$ e $g$ são de classe $C^{2}$ temos que $N(u, v)=$ $(f(u()),. g(v())$.$) é absolutamente contínua, e sua derivada \frac{d}{d x} N(u, v)=\left(f^{\prime}(u) u^{\prime}, g^{\prime}(v) v^{\prime}\right)$ satisfaz

$$
\begin{aligned}
\int_{0}^{1}\left(\left|f^{\prime}(u(x))\right|^{2}\left|u^{\prime}(x)\right|^{2}+\left|g^{\prime}(v(x))\right|^{2}\left|v^{\prime}(x)\right|^{2}\right) d x & \leq \sup _{x \in[0,1]}\left\{\left|f^{\prime}(u(x))\right|^{2},\left|g^{\prime}(v(x))\right|^{2}\right\}\|(u, v)\|_{H}^{2} \\
& \leq k\left(\|(u, v)\|_{H}\right)\|(u, v)\|_{H}^{2},
\end{aligned}
$$

onde $k(\|(u, v \|))$ é uma constante que depende de $\|(u, v)\|_{H}$. Isto mostra que $N: H \rightarrow$ $H^{1}(0,1)^{2}$ é uma aplicação limitada. Como conjuntos limitados de $H^{1}(0,1)^{2}$ são precompactos em $L^{2}(0,1)^{2}$, temos que $N: H \rightarrow X$ é compacta, o que conclui a demonstração do lema.

Pelo fato de $N: H \rightarrow X$ ser de classe $C^{1,1}$ obtemos existência e unicidade de solução local para o problema (1.1) com dados iniciais em $H$ e então temos um $C^{1,1}$-semigrupo 
local $T(t)$ de operadores não lineares em $H$. Mostraremos a seguir, que $T(t)$ é de fato, um semigrupo, isto é, mostraremos que as soluções estão definidas em $[0,+\infty)$.

Para $\left(u_{0}, v_{0}\right) \in H$, considere a solução maximal $(u(., t), v(., t))=T(t)\left(u_{0}, v_{0}\right)$ cujo domínio de definição é dado por $\left[0, s\left(u_{0}, v_{0}\right)\right)$, onde $0<s\left(u_{0}, v_{0}\right) \leq+\infty$. Como $N$ é Lipschitz contínua em conjuntos limitados de $H$ segue do Teorema 3.3.4 de [11] que, se $(u(., t), v(., t))$ é limitada em $H, s\left(u_{0}, v_{0}\right)=+\infty$. Provemos então que $(u(., t), v(., t))$ é limitada em $H$. Para tal, considere o seguinte funcional em $H$

$$
V(\varphi, \psi)=\frac{1}{2} \int_{0}^{1}\left[a(x) \varphi^{\prime}(x)^{2}+b(x) \psi^{\prime}(x)^{2}-2 F(\varphi(x))-2 G(\psi(x))\right] d x,
$$

onde $F(u)=\int_{0}^{u} f(s) d s$ e $G(v)=\int_{0}^{v} g(s) d s$. Calculando o funcional $V$ em $(u(., t), v(., t))$ e derivando com relação a $t$ obtemos

$$
\begin{aligned}
\frac{d}{d t} V(u(., t), v(., t)) & =\int_{0}^{1}\left[a(x) u_{x} u_{x t}+b(x) v_{x} v_{x t}-f(u) u_{t}-g(v) v_{t}\right] d x \\
& =\left.\left[a(x) u_{x} u_{t}+b(x) v_{x} v_{t}\right]\right|_{0} ^{1}-\int_{0}^{1}\left(u_{t}^{2}+v_{t}^{2}\right) d x \\
& =u_{t}(0, t)\left[a(0) u_{x}(0, t)+b(0) v_{x}(0, t)\right]-\int_{0}^{1}\left(u_{t}^{2}+v_{t}^{2}\right) d x \\
& =-\int_{0}^{1}\left(u_{t}^{2}+v_{t}^{2}\right) d x \leq 0
\end{aligned}
$$

e, portanto, para todo $t \in\left[0, s\left(u_{0}, v_{0}\right)\right)$, temos

$$
V(u(., t), v(., t)) \leq V\left(u_{0}, v_{0}\right) .
$$

Para qualquer $\varepsilon>0$, a hipótese (H2) nos garante a existência de constantes $c_{1 \varepsilon}$ e $c_{2 \varepsilon}$ tais que

$$
\begin{gathered}
F(u)-\varepsilon u^{2}=\int_{0}^{u}\left[\frac{f(s)}{s}-2 \varepsilon\right] s d s \leq c_{1 \varepsilon}, \\
G(v)-\varepsilon v^{2}=\int_{0}^{v}\left[\frac{g(s)}{s}-2 \varepsilon\right] s d s \leq c_{2 \varepsilon},
\end{gathered}
$$

para todos $u, v \in \mathbb{R}$. Então, para $(\varphi, \psi) \in H$, temos

$$
\begin{aligned}
V(\varphi, \psi) & \geq \frac{1}{2} \min _{x \in[0,1]}\{a(x), b(x)\}\|(\varphi, \psi)\|_{H}^{2}-\varepsilon \int_{0}^{1}\left[\varphi(x)^{2}+\psi(x)^{2}\right] d x-\left(c_{1 \varepsilon}+c_{2 \varepsilon}\right) \\
& \geq \frac{k_{2}}{2}\|(\varphi, \psi)\|_{H}^{2}-\varepsilon\|(\varphi, \psi)\|_{X}^{2}-c_{\varepsilon} \\
& \geq\left(\frac{k_{2}}{2}-\varepsilon\right)\|(\varphi, \psi)\|_{H}^{2}-c_{\varepsilon},
\end{aligned}
$$


onde $k_{2}=\min _{x \in[0,1]}\{a(x), b(x)\}>0$ e $c_{\varepsilon}=c_{1 \varepsilon}+c_{2 \varepsilon}$. Tomando $\varepsilon=k_{2} / 4$ segue que

$$
\|(\varphi, \psi)\|_{H}^{2} \leq \frac{4}{k_{2}} V(\varphi, \psi)+c_{\varepsilon}
$$

para todo $(\varphi, \psi) \in H$. Aplicando esta desigualdade para $(u(., t), v(., t))$ e usando (1.3) obtemos

$$
\begin{aligned}
\|(u(., t), v(., t))\|_{H}^{2} & \leq \frac{4}{k_{2}} V(u(., t), v(., t))+c_{\varepsilon} \\
& \leq \frac{4}{k_{2}} V\left(u_{0}, v_{0}\right)+c_{\varepsilon}
\end{aligned}
$$

para todo $t \in\left[0, s\left(u_{0}, v_{0}\right)\right)$, o que implica que $s\left(u_{0}, v_{0}\right)=+\infty$.

Para qualquer $r>0$, se $\left\|\left(u_{0}, v_{0}\right)\right\|_{H} \leq r$ então $\left|u_{0}(x)\right|,\left|v_{0}(x)\right| \leq r$, para todo $x \in[0,1]$, e

$$
\begin{aligned}
V\left(u_{0}, v_{0}\right) & \leq \frac{1}{2} \max _{x \in[0,1]}\{a(x), b(x)\}\left\|\left(u_{0}, v_{0}\right)\right\|_{H}^{2}+ \\
& +\int_{0}^{1}\left[\left|\int_{0}^{u_{0}(x)}\right| f(s)|d s|+\left|\int_{0}^{v_{0}(x)}\right| g(s)|d s|\right] d x \\
& \leq \frac{k_{1}}{2}\left\|\left(u_{0}, v_{0}\right)\right\|_{H}^{2}+c(r)\left\|\left(u_{0}, v_{0}\right)\right\|_{X}^{2} \\
& \leq \frac{k_{1}}{2} r^{2}+c(r) r^{2} \equiv C(r) .
\end{aligned}
$$

Como $V$ é não-crescente ao longo das soluções, concluímos que órbitas de conjuntos limitados são limitadas, pois

$$
\begin{aligned}
\left\|T(t)\left(u_{0}, v_{0}\right)\right\|_{H}^{2} & \leq \frac{4}{k_{2}} V\left(T(t)\left(u_{0}, v_{0}\right)\right)+c_{\varepsilon} \\
& \leq \frac{4}{k_{2}} V\left(u_{0}, v_{0}\right)+c_{\varepsilon} \\
& \leq \frac{4}{k_{2}} C(r)+c_{\varepsilon} .
\end{aligned}
$$

Agora, vejamos que o operador $A$ tem resolvente compacto. Do Teorema de LaxMilgram e do fato de $A$ ser positivo temos a existência de $A^{-1}$. Como $A$ é fechado, $A^{-1}$ também o é. Segue então do Teorema do Gráfico Fechado que $A^{-1}: X \rightarrow X^{1}=D(A) \subset$ $X$ é contínuo. Assim, se $B \subset X$ é um subconjunto limitado, então $A^{-1}(B)$ é limitado em $X^{1}=D(A)$. Como $X^{1}$ está compactamente imerso em $X, A^{-1}(B)$ é pré-compacto em $X$. Portanto, $A^{-1}$ é um operador compacto e, consequentemente, o operador $(\lambda-A)^{-1}$ é compacto para todo $\lambda \in \rho(A)$, resolvente do operador $A$. 
Da fórmula da variação das constantes, segue que $T(t)$ é completamente contínuo para $t>0$. Em particular, a órbita positiva $\gamma^{+}\left(u_{0}, v_{0}\right)$ de $\left(u_{0}, v_{0}\right) \in H$ é pré-compacta, tem conjunto $\omega$-limite não-vazio, compacto, conexo e invariante.

O funcional $V$ definido anteriormente é um funcional de Liapunov para $T(t)$ pois:

(i) por (1.6), $V(\varphi, \psi)$ é limitado inferiormente;

(ii) ainda por (1.6), $V(\varphi, \psi) \rightarrow+\infty$ quando $\|(\varphi, \psi)\|_{H} \rightarrow+\infty$;

(iii) segue de (1.2) que $V\left(T(t)\left(u_{0}, v_{0}\right)\right)$ é não-crescente em $t$, para cada $\left(u_{0}, v_{0}\right) \in H$;

(iv) se $V\left(T(t)\left(u_{0}, v_{0}\right)\right)=V\left(u_{0}, v_{0}\right)$, segue de (1.2) que $\left(u_{0}, v_{0}\right) \in E=\{$ conjunto de pontos de equilíbrio de $T(t)\}$.

Dessa forma, como toda órbita positiva é pré-compacta, o semigrupo $\{T(t): H \rightarrow$ $H, t \geq 0\}$ é um sistema gradiente, o que implica que o conjunto $\omega$-limite de qualquer ponto, $\omega\left(u_{0}, v_{0}\right)$, está contido em $E$.

Vejamos a seguir que o conjunto $E$ dos pontos de equilíbrio de $T(t)$ é limitado, o que implica que $T(t)$ é ponto dissipativo. Seja $(\varphi, \psi) \in H$ um ponto de equilíbrio de $T(t)$, então $(\varphi, \psi)$ satisfaz

$$
\left\{\begin{array}{l}
\left(a(x) \varphi^{\prime}(x)\right)^{\prime}+f(\varphi(x))=0, \quad x \in(0,1) \\
\left(b(x) \psi^{\prime}(x)\right)^{\prime}+g(\psi(x))=0, \quad x \in(0,1) \\
\varphi(0)=\psi(0) \\
a(0) \varphi^{\prime}(0)+b(0) \psi^{\prime}(0)=0 \\
\varphi(1)=\psi(1)=0 .
\end{array}\right.
$$

Multiplicando a primeira equação por $\varphi(x)$, a segunda por $\psi(x)$, integrando em $[0,1]$ e somando as expressões resultantes obtemos

$$
\int_{0}^{1}\left[a(x) \varphi^{\prime}(x)^{2}+b(x) \psi^{\prime}(x)^{2}\right] d x=\int_{0}^{1}[f(\varphi(x)) \varphi(x)+g(\psi(x)) \psi(x)] d x
$$

Como $f$ e $g$ satisfazem $(H 2)$ temos que, dado $\varepsilon>0$ existe uma constante $M=M(\varepsilon)>0$ tal que $f(u) / u<\varepsilon$ e $g(v) / v<\varepsilon$ sempre que $|u|,|v|>M$. Considere então, os intervalos 


$$
\begin{aligned}
I_{1, \theta}=\{x \in[0,1]:|\theta(x)|>M\} \text { e } I_{2, \theta}= & {[0,1] \backslash I_{1, \theta}, \text { onde } \theta \in\{\varphi, \psi\} . \text { Dessa forma } } \\
\int_{0}^{1}\left[a(x) \varphi^{\prime}(x)^{2}+b(x) \psi^{\prime}(x)^{2}\right] d x= & \int_{I_{1, \varphi}} f(\varphi(x)) \varphi(x) d x+\int_{I_{1, \psi}} g(\psi(x)) \psi(x) d x+ \\
& +\int_{I_{2, \varphi}} f(\varphi(x)) \varphi(x) d x+\int_{I_{2, \psi}} g(\psi(x)) \psi(x) d x \\
\leq & \varepsilon\|(\varphi, \psi)\|_{X}^{2}+K,
\end{aligned}
$$

onde a constante $K=M \sup _{|s| \leq M}\{|f(s)|,|g(s)|\}$. Sendo $k_{2}=\min _{x \in[0,1]}\{a(x), b(x)\}$ e escolhendo $\varepsilon=k_{2} / 2$ concluímos que

$$
\|(\varphi, \psi)\|_{H}^{2} \leq \frac{2 K}{k_{2}}
$$

Definição 1.1 Seja $(\varphi, \psi)$ um ponto de equilíbrio de (1.1). Definimos as variedades estável e instável de $(\varphi, \psi)$ como sendo os conjuntos

$$
\begin{gathered}
W^{s}(\varphi, \psi)=\left\{\left(u_{0}, v_{0}\right) \in X^{\alpha}: T(t)\left(u_{0}, v_{0}\right) \rightarrow(\varphi, \psi) \text { em } X^{\alpha} \text { quando } t \rightarrow+\infty\right\} \\
W^{u}(\varphi, \psi)=\left\{\left(u_{0}, v_{0}\right) \in X^{\alpha}: T(t)\left(u_{0}, v_{0}\right) \text { está definida para } t \leq 0\right. \text { e } \\
\left.T(t)\left(u_{0}, v_{0}\right) \rightarrow(\varphi, \psi) \text { em } X^{\alpha} \text { quando } t \rightarrow-\infty\right\} .
\end{gathered}
$$

Definição 1.2 Dizemos que $(\varphi, \psi) \in E$ é hiperbólico se o espectro $\sigma\left(-A+N^{\prime}(\varphi, \psi)\right)$ é disjunto do eixo imaginário, isto é, $\sigma(-A+n \varphi, \psi)) \cap i \mathbb{R}=\emptyset$.

Já vimos que o semigrupo $T(t)$ é um sistema gradiente, completamente contínuo para $t>0$ e também, que $T(t)$ é ponto dissipativo; segue-se então do Teorema 3.8 .5 de [8] o seguinte resultado

Teorema 1.1 Suponha que $f$ e g satisfaçam as hipóteses $(H 1)$ e (H2). Então $(0.1)$ - (0.3) define um $C^{1,1}$-semigrupo gradiente $T(t)$ em $H$, o conjunto de equilíbrios $E$ é limitado e existe um atrator global conexo $\mathcal{A}=\mathcal{A}_{a, b, f, g} \subset H$. Além disso, se cada elemento $(\varphi, \psi) \in E$ é hiperbólico, então

$$
\mathcal{A}=\bigcup_{(\varphi, \psi) \in E} W^{u}(\varphi, \psi) .
$$

O próximo teorema é um resultado da teoria geral de equações diferenciais parabólicas, que se encontra em Henry [11] (Teorema 5.2.1). Ele nos dá uma caracterização das variedades locais estável e instável de um ponto de equilíbrio. 
Teorema 1.2 Sejam A um operador setorial em um espaço de Banach $X$ e $f: U \rightarrow X$, onde $U$ é uma vizinhança aberta de $x_{0}$ em $X^{\alpha}$, para $\alpha<1$ e $x_{0}$ um ponto de equilíbrio da equação

$$
\frac{d x}{d t}+A x=f(x)
$$

Suponha que $f\left(x_{0}+z\right)=f\left(x_{0}\right)+B z+g(z)$, onde $B \in \mathcal{L}\left(X^{\alpha}, X\right), g(0)=0$ e $\| g\left(z_{1}\right)-$ $g\left(z_{2}\right)\|\leq k(\rho)\| z_{1}-z_{2} \|_{\alpha}$, para $\left\|z_{1}\right\|_{\alpha},\left\|z_{2}\right\|_{\alpha} \leq \rho$, onde $k(\rho) \rightarrow 0$ quando $\rho \rightarrow 0$; podemos supor $k($.$) não - decrescente.$

Suponha também que, para $L=A-B$, o espectro $\sigma(L)$ é disjunto do eixo imaginário. Sejam $X=X_{1} \oplus X_{2}$ e $L=L_{1} \oplus L_{2}$, de modo que $L_{1}=\left.L\right|_{X_{1}}$ tem espectro $\sigma_{1}=\{\lambda \in$ $\sigma(L): \operatorname{Re} \lambda<0\}$ e $L_{2}=\left.L\right|_{X_{2}}$ tem espectro $\sigma_{2}=\{\lambda \in \sigma(L): \operatorname{Re} \lambda>0\}$. Sejam $E_{1}$ e $E_{2}$ as projeções sobre $X_{1}$ e $X_{2}$, respectivamente. Então existem $\rho>0$ e $M \geq 1$ tais que

(i) A variedade estável local $W_{\mathrm{loc}}^{s}=W^{s}\left(t_{0}, \rho\right)$ é

$$
W_{\mathrm{loc}}^{s}=\left\{z_{0}:\left\|E_{2} z_{0}\right\|_{\alpha} \leq \frac{\rho}{2 M},\left\|z\left(t ; t_{0}, z_{0}\right)\right\|_{\alpha} \leq \rho, \text { para todo } t \geq t_{0}\right\}
$$

e $\left.E_{2}\right|_{W_{\text {loc }}^{s}}: W_{\text {loc }}^{s} \rightarrow \bar{B}_{X_{2}^{\alpha}}\left(0, \frac{\rho}{2 M}\right)$ é um homeomorfismo. Além disso, $W_{\text {loc }}^{s}$ é tangente $a X_{2}^{\alpha}$ na origem e se $z_{0} \in W_{\text {loc }}^{s}$, então $\left\|z\left(t ; t_{0}, z_{0}\right)\right\|_{\alpha} \rightarrow 0$ quando $t \rightarrow+\infty$.

(ii) A variedade instável local $W_{\mathrm{loc}}^{u}=W^{u}\left(t_{0}, \rho\right)$ é

$$
\begin{aligned}
W_{\mathrm{loc}}^{u}= & \left\{z_{0}:\left\|E_{1} z_{0}\right\|_{\alpha} \leq \frac{\rho}{2 M}, z\left(t ; t_{0}, z_{0}\right) \text { é uma solução sobre }\left(-\infty, t_{0}\right),\right. \\
& \left.\left\|z\left(t ; t_{0}, z_{0}\right)\right\|_{\alpha} \leq \rho, \text { para todo } t \leq t_{0}\right\}
\end{aligned}
$$

e $\left.E_{1}\right|_{W_{\text {loc }}^{u}}: W_{\text {loc }}^{u} \rightarrow \bar{B}_{X_{1}}\left(0, \frac{\rho}{2 M}\right)$ é um homeomorfismo. Além disso, $W_{\text {loc }}^{u}$ é tangente a $X_{1}$ na origem e se $z_{0} \in W_{\mathrm{loc}}^{u}$, então $z\left(t ; t_{0}, z_{0}\right) \rightarrow 0$ quando $t \rightarrow-\infty$.

(iii) $S e\left\|E_{1,2}\right\|_{\alpha} \leq \frac{\rho}{2 M} e\left\|z\left(t ; t_{0}, z_{0}\right)\right\|_{\alpha} \leq \rho$, para todo $t \geq t_{0}$ ou $t \leq t_{0}$, então $z_{0} \in$ $W_{\text {loc }}^{u} \cup W_{\text {loc }}^{s}$.

Na prova deste teorema, Henry [11] obtém as seguintes caracterizações dos espaços tangentes às variedades estável e instável.

Teorema 1.3 Sejam $A, f$ e $x_{0}$ como no teorema anterior, além disso, suponha que $f$ é uma aplicação de classe $C^{1}$. Considere a equação linearizada de (1.8) em torno de $x_{0}$,

$$
\frac{d y}{d t}=\left(f^{\prime}\left(x_{0}\right)-A\right) y
$$


Se $z_{0} \in W^{s}\left(x_{0}\right)$, então

$$
\begin{aligned}
T_{z_{0}} W^{s}\left(x_{0}\right)= & \left\{y\left(t_{0}\right): y \text { é solução de }(1.9) \text { em } t_{0}<t<+\infty \text { e }\|y(t)\|_{X}=O\left(e^{-\epsilon t}\right)\right. \\
& \text { quando } t \rightarrow+\infty, \text { para algum } \epsilon>0\} .
\end{aligned}
$$

Se $z_{0} \in W^{u}\left(x_{0}\right)$, então

$$
\begin{aligned}
T_{z_{0}} W^{u}\left(x_{0}\right)= & \left\{y\left(t_{0}\right): y \text { é solução de }(1.9) \text { em }-\infty<t<t_{0} \text { e }\|y(t)\|_{X}=O\left(e^{\epsilon t}\right)\right. \\
& \text { quando } t \rightarrow-\infty, \text { para algum } \epsilon>0\}
\end{aligned}
$$

\subsection{Propriedade de Sturm-Liouville}

Nesta seção, demonstramos algumas propriedades da teoria clássica de Sturm-Liouville para o operador linearizado em torno de um equilíbrio, o que nos permitirá concluir, através de um resultado de [9], que o conjunto $\omega$-limite de qualquer ponto consiste apenas de um único ponto de equilíbrio.

Seja $(u(x, t), v(x, t)), x \in[0,1], t \geq 0$, uma solução do problema (0.1)-(0.3) e considere a função $w$ definida em $[-1,1] \times[0, \infty)$ por

$$
w(x, t)=\left\{\begin{array}{l}
u(-x, t), \quad x \in[-1,0], t \geq 0 \\
v(x, t), \quad x \in[0,1], t \geq 0
\end{array}\right.
$$

É fácil ver que $w$ é solução do seguinte problema

$$
\left\{\begin{array}{l}
w_{t}=\left(a(-x) w_{x}\right)_{x}+f(w), x \in(-1,0), t>0 \\
w_{t}=\left(b(x) w_{x}\right)_{x}+g(w), \quad x \in(0,1), t>0 \\
w(0-, t)=w(0+, t) \\
a(0) w_{x}(0-, t)=b(0) w_{x}(0+, t) \\
w(-1, t)=w(1, t)=0
\end{array}\right.
$$

Reciprocamente, a toda solução de (1.12) associamos uma solução de (0.1)-(0.3) da forma óbvia. Reescrevemos (1.12) como a equação de evolução

$$
w_{t}=-\tilde{A} w+\tilde{N} w, \quad t>0
$$


onde $\tilde{A}: D(\tilde{A}) \subset L^{2}(-1,1) \rightarrow L^{2}(-1,1)$ é o operador não limitado fechado positivo autoadjunto definido por

$$
\tilde{A} w(x)=\left\{\begin{array}{l}
-\left(a(-x) w_{x}(x)\right)_{x}, \quad x \in(-1,0), \\
-\left(b(x) w_{x}(x)\right)_{x}, \quad x \in(0,1)
\end{array}\right.
$$

$D(\tilde{A})=\left\{\phi \in H_{0}^{1}((-1,1)) \cap H^{2}((-1,0) \cup(0,1)): a(0) \phi^{\prime}(0-)=b(0) \phi^{\prime}(0+)\right\}$ e a aplicação não-linear $\tilde{N}: \tilde{X}^{\alpha} \rightarrow \tilde{X}=L^{2}(-1,1)$ é definida por

$$
\tilde{N} w(x)= \begin{cases}f(w(x)), & x \in(-1,0), \\ g(w(x)), & x \in(0,1),\end{cases}
$$

onde $\tilde{X}^{\alpha}, \alpha \geq 0$, são os espaços de potências fracionárias definidos pelo operador setorial $\tilde{A}$. Como na primeira seção, o operador $\tilde{A}$ está associado à forma bilinear $\tilde{\tau}$ definida em $H_{0}^{1}(-1,1) \times H_{0}^{1}(-1,1)$ por

$$
\tilde{\tau}(w, z)=\int_{-1}^{0} a(-x) w^{\prime} z^{\prime} d x+\int_{0}^{1} b(x) w^{\prime} z^{\prime} d x
$$

para todos $w, z \in H_{0}^{1}(-1,1)$. Dessa forma, temos $\tilde{X}^{1 / 2}=H_{0}^{1}(-1,1)$. No restante desta seção, consideraremos $\tilde{N}$ com domínio $H_{0}^{1}(-1,1)$ e os dados iniciais serão tomados nesse espaço.

Nosso objetivo, a partir de agora, é demonstrar algumas propriedades análogas às da teoria clássica de Sturm-Liouville para o operador $\tilde{A}-\tilde{N}^{\prime}(\varphi)$, quando $\varphi \in \tilde{E}=\{$ conjunto dos pontos de equilíbrio de $(1.12)\} \subset H_{0}^{1}(-1,1)$. Se $\varphi \in \tilde{E}$, então $\varphi$ satisfaz

$$
\left\{\begin{array}{l}
\left(a(-x) \varphi^{\prime}\right)^{\prime}+f(\varphi)=0, \quad x \in(-1,0) \\
\left(b(x) \varphi^{\prime}\right)^{\prime}+g(\varphi)=0, \quad x \in(0,1) \\
\varphi(0-)=\varphi(0+) \\
a(0) \varphi^{\prime}(0-)=b(0) \varphi^{\prime}(0+) \\
\varphi(-1)=\varphi(1)=0 .
\end{array}\right.
$$

O problema de autovalor para a linearização de (1.14) em torno de $\varphi$ é

$$
\left\{\begin{array}{l}
\left(p(x) u^{\prime}\right)^{\prime}+(c(x)-\lambda) u=0, \quad x \in(-1,0) \cup(0,1) \\
u(0-)=u(0+) \\
g(0-) u^{\prime}(0-)=g(0+) u^{\prime}(0+) \\
u(-1)=u(1)=0
\end{array}\right.
$$


ond e $p(x)=\left\{\begin{array}{l}a(-x), x \in(-1,0) \\ b(x), x \in(0,1)\end{array}\right.$ e $c(x)=\left\{\begin{array}{l}f^{\prime}(\varphi(x)), x \in(-1,0) \\ g^{\prime}(\varphi(x)), x \in(0,1)\end{array}\right.$.

Observemos que $\varphi$ é contínua em $x=0$, porém a função $c$ não está definida em $x=0$, mas existem e são finitos os limites laterais $c(0-)$ e $c(0+)$. O mesmo comentário pode ser feito para a função $g$.

Iniciemos nosso estudo com alguns resultados gerais sobre as seguintes equações diferenciais

$$
\left\{\begin{array}{l}
\left(p_{1}(x) u^{\prime}\right)^{\prime}-c_{1}(x) u=0, x \in(-1,0) \cup(0,1) \\
u(0-)=u(0+) \\
p_{1}(0-) u^{\prime}(0-)=p_{1}(0+) u^{\prime}(0+)
\end{array}\right.
$$

e

$$
\left\{\begin{array}{l}
\left(p_{2}(x) v^{\prime}\right)^{\prime}-c_{2}(x) v=0, \quad x \in(-1,0) \cup(0,1) \\
v(0-)=v(0+) \\
p_{2}(0-) v^{\prime}(0-)=p_{2}(0+) v^{\prime}(0+)
\end{array}\right.
$$

onde $p_{j}(x)=\left\{\begin{array}{l}a_{j}(-x), x \in(-1,0) \\ b_{j}(x), x \in(0,1)\end{array}, j=1,2\right.$, com $a_{j}, b_{j} \in C^{2}([0,1],(0, \infty))$, e $c_{1}, c_{2}$ são funções reais e contínuas em $(-1,0) \cup(0,1)$.

Lema 1.2 Sejam u e v soluções não - nulas de (1.16) e (1.17), respectivamente. Então, para $x \in(-1,0) \cup(0,1)$ tal que $v(x) \neq 0$ temos

$$
\frac{d}{d x}\left[\frac{u}{v}\left(p_{1} u_{x} v-p_{2} u v_{x}\right)\right]=\left(c_{1}-c_{2}\right) u^{2}+\left(p_{1}-p_{2}\right) u_{x}^{2}+p_{2}\left(\frac{u_{x} v-u v_{x}}{v}\right)^{2}
$$

Demonstração. Usando o fato que $u$ e $v$ são soluções de (1.16) e (1.17), respectivamente, 
obtemos

$$
\begin{aligned}
\frac{d}{d x}\left[\frac{u}{v}\left(p_{1} u_{x} v-p_{2} u v_{x}\right)\right]= & \left(\frac{u}{v}\right)_{x}\left(p_{1} u_{x} v-p_{2} u v_{x}\right)+ \\
& +\frac{u}{v}\left[\left(p_{1} u_{x}\right)_{x} v+p_{1} u_{x} v_{x}-\left(p_{2} v_{x}\right)_{x} u-p_{2} v_{x} u_{x}\right] \\
= & \left(\frac{u_{x} v}{v^{2}}-\frac{u v_{x}}{v^{2}}\right)\left(p_{1} u_{x} v-p_{2} u v_{x}\right)+ \\
& +\frac{u}{v}\left[c_{1} u v+p_{1} u_{x} v_{x}-c_{2} v u-p_{2} v_{x} u_{x}\right] \\
= & p_{1} u_{x}^{2}-\frac{p_{1} u u_{x} v_{x}}{v}-\frac{p_{2} u u_{x} v_{x}}{v}+\frac{p_{2} u^{2} v_{x}^{2}}{v^{2}}+ \\
& +c_{1} u^{2}-c_{2} u^{2}+\frac{p_{1} u u_{x} v_{x}}{v}-\frac{p_{2} u u_{x} v_{x}}{v} \\
= & \left(c_{1}-c_{2}\right) u^{2}+p_{1} u_{x}^{2}+\frac{p_{2} u^{2} v_{x}^{2}}{v^{2}}-\frac{2 p_{2} u_{x} u v_{x}}{v} \pm p_{2} u_{x}^{2} \\
= & \left(c_{1}-c_{2}\right) u^{2}+\left(p_{1}-p_{2}\right) u_{x}^{2}+p_{2}\left(u_{x}-\frac{u v_{x}}{v}\right)^{2} .
\end{aligned}
$$

O lema a seguir compara a distribuição dos zeros das soluções das equações (1.16) e (1.17).

Lema 1.3 Sejam $p_{1}, p_{2}, c_{1}, c_{2}$ funções reais, onde $c_{1}, c_{2}$ são contínuas e limitadas em $(-1,0) \cup(0,1), p_{1}, p_{2}$ são positivas em $(-1,0) \cup(0,1)$ com

$$
p_{1}(x) \geq p_{2}(x)>\delta>0, \quad c_{1}(x) \geq c_{2}(x), x \in(-1,0) \cup(0,1) .
$$

Suponha também que a identidade $c_{1} \equiv c_{2}$ não seja satisfeita em qualquer subintervalo de $(-1,0) \cup(0,1)$. Se u e v são soluções não - nulas de (1.16) e (1.17), respectivamente, então em cada componente de $C=\{x \in(-1,1): u(x) \neq 0\}$ existe um zero de $v$.

Demonstração. Seja $\left(x_{1}, x_{2}\right)$ uma componente conexa de $C$, isto é, $u\left(x_{1}\right)=u\left(x_{2}\right)=0$ e $u(x) \neq 0$, para todo $x \in\left(x_{1}, x_{2}\right)$.

Primeiramente, suponhamos por absurdo, que $v$ não tenha nenhum zero em $\left[x_{1}, x_{2}\right]$. Não há perda de generalidade em supor que $u$ e $v$ são positivas no intervalo $\left(x_{1}, x_{2}\right)$. Temos alguns casos a considerar:

Caso 1: $0 \notin\left[x_{1}, x_{2}\right]$

Integrando (1.18) em $\left[x_{1}, x_{2}\right]$ obtemos

$$
\int_{x_{1}}^{x_{2}} \frac{d}{d x}\left[\frac{u}{v}\left(p_{1} u_{x} v-p_{2} u v_{x}\right)\right] d x=\int_{x_{1}}^{x_{2}}\left[\left(c_{1}-c_{2}\right) u^{2}+\left(p_{1}-p_{2}\right) u_{x}^{2}+p_{2}\left(\frac{u_{x} v-u v_{x}}{v}\right)^{2}\right] d x
$$




$$
0=\int_{x_{1}}^{x_{2}}\left[\left(c_{1}-c_{2}\right) u^{2}+\left(p_{1}-p_{2}\right) u_{x}^{2}+p_{2}\left(\frac{u_{x} v-u v_{x}}{v}\right)^{2}\right] d x
$$

mas pelas hipóteses assumidas o lado direito da expressão acima é positivo, donde temos uma contradição .

Caso 2: $0 \in\left[x_{1}, x_{2}\right]$

Suponhamos que $0 \in\left(x_{1}, x_{2}\right)$; o caso em que $x=0$ é um dos extremos é análogo. Para $\varepsilon>0$ suficientemente pequeno, integramos (1.18) em $\left(x_{1},-\varepsilon\right) \cup\left(\varepsilon, x_{2}\right)$ e obtemos

$$
\begin{aligned}
\left\{\int_{x_{1}}^{-\varepsilon}+\int_{\varepsilon}^{x_{2}}\right\} \frac{d}{d x}\left[\frac{u}{v}\left(p_{1} u_{x} v-p_{2} u v_{x}\right)\right]= & \left\{\int_{x_{1}}^{-\varepsilon}+\int_{\varepsilon}^{x_{2}}\right\}\left[\left(c_{1}-c_{2}\right) u^{2}+\left(p_{1}-p_{2}\right) u_{x}^{2}+\right. \\
& \left.+p_{2}\left(\frac{u_{x} v-u v_{x}}{v}\right)^{2}\right] .
\end{aligned}
$$

Fazendo $\varepsilon \rightarrow 0$,

$$
\begin{aligned}
\left(\frac{u}{v}\left(p_{1} u_{x} v-p_{2} u v_{x}\right)\right)(0-)-\left(\frac{u}{v}\left(p_{1} u_{x} v-p_{2} u v_{x}\right)\right)(0+)= & \int_{x_{1}}^{x_{2}}\left[\left(c_{1}-c_{2}\right) u^{2}+\left(p_{1}-p_{2}\right) u_{x}^{2}+\right. \\
& \left.+p_{2}\left(\frac{u_{x} v-u v_{x}}{v}\right)^{2}\right] d x>0
\end{aligned}
$$

e utilizando as condições de $u$ e $v$ em $x=0$ temos

$$
0=\int_{x_{1}}^{x_{2}}\left[\left(c_{2}-c_{1}\right) u^{2}+\left(p_{1}-p_{2}\right) u_{x}^{2}+p_{2}\left(\frac{u_{x} v-u v_{x}}{v}\right)^{2}\right] d x>0
$$

o que é um absurdo.

Para completar a prova, resta analisar o caso em que $v\left(x_{1}\right)=0$ ou $v\left(x_{2}\right)=0$. Por exemplo, analisemos o caso em que $v\left(x_{1}\right)=0$ e $v\left(x_{2}\right) \neq 0$. Dessa forma, $v_{x}\left(x_{1}\right) \neq 0$ e

$$
\lim _{x \rightarrow x_{1}}\left[\frac{u}{v}\left(p_{1} u_{x} v-p_{2} u v_{x}\right)\right]=\lim _{x \rightarrow x_{1}}\left[-\frac{p_{2} u^{2} v_{x}}{v}\right], \text { pois } u\left(x_{1}\right)=0
$$

Usando a Regra de L'Hospital concluímos que

$$
\lim _{x \rightarrow x_{1}}\left[-\frac{p_{2} u^{2} v_{x}}{v}\right]=\lim _{x \rightarrow x_{1}}\left[\frac{-2 p_{2} u u_{x} v_{x}-\left(p_{2} v_{x}\right)_{x} u^{2}}{v_{x}}\right]=\lim _{x \rightarrow x_{1}}\left[-2 p_{2} u u_{x}+\frac{c_{2} v u^{2}}{v_{x}}\right]=0 .
$$

Ou seja, podemos integrar (1.18) em $\left[x_{1}+\delta, x_{2}\right]$ e fazer $\delta \rightarrow 0$ para obtermos uma contradição, mesmo quando $x_{1}$ for também um zero de $v$. 
Consideremos agora os seguintes problemas de valor inicial determinados pelas equações diferenciais (1.16) e (1.17)

$$
\left\{\begin{array}{l}
\left(p_{1}(x) u^{\prime}\right)^{\prime}-c_{1}(x) u=0, \quad x \in(-1,0) \cup(0,1) \\
u(0-)=u(0+) \\
p_{1}(0-) u^{\prime}(0-)=p_{1}(0+) u^{\prime}(0+) \\
u(-1)=0, u^{\prime}(-1)=1
\end{array}\right.
$$

e

$$
\left\{\begin{array}{l}
\left(p_{2}(x) v^{\prime}\right)^{\prime}-c_{2}(x) v=0, \quad x \in(-1,0) \cup(0,1) \\
v(0-)=v(0+) \\
p_{2}(0-) v^{\prime}(0-)=p_{2}(0+) v^{\prime}(0+) \\
v(-1)=0, \quad v^{\prime}(-1)=1 .
\end{array}\right.
$$

Corolário 1.1 Com as mesmas hipóteses do lema anterior, sejam u e v soluções não nulas dos problemas (1.19) e (1.20), respectivamente, e suponha que u possui $n$ zeros no intervalo $(-1,1]$. Então $v$ tem pelo menos $n$ zeros no mesmo intervalo, sendo o i-ésimo zero de $v$ menor do que o i-ésimo zero de $u$.

Demonstração. Segue diretamente do lema anterior e do fato de $u(-1)=0$.

Teorema 1.4 Sejam $a, b \in C^{1}([0,1] ;(0, \infty))$ e $c \in C((-1,0) \cup(0,1) \times \mathbb{R} ; \mathbb{R})$. Suponhamos que c é decrescente em $\lambda$, para cada $x \in(-1,0) \cup(0,1)$ e que c é limitada em $(-1,0) \cup(0,1)$, para cada $\lambda \in \mathbb{R}$. Sendo $\Omega=(-1,0) \cup(0,1)$, suponhamos ainda que

(i) $\xi(\lambda)=\inf _{x \in \Omega} c(x, \lambda) \longrightarrow+\infty$ quando $\lambda \rightarrow-\infty$;

(ii) $\eta(\lambda)=\sup _{x \in \Omega} c(x, \lambda) \longrightarrow-\infty$ quando $\lambda \rightarrow+\infty$.

Seja

$$
p(x)=\left\{\begin{array}{l}
a(-x), x \in(-1,0) \\
b(x), x \in(0,1)
\end{array}\right.
$$

e considere o problema de valor de fronteira

$$
\left\{\begin{array}{l}
\left(p(x) u^{\prime}\right)^{\prime}+c(x, \lambda) u=0, \quad x \in(-1,0) \cup(0,1) \\
u(0-)=u(0+) \\
p(0-) u^{\prime}(0-)=p(0+) u^{\prime}(0+) \\
u(-1)=0, \quad u(1)=0 .
\end{array}\right.
$$


Então, existe uma sequência decrescente de números reais, $\lambda_{1}>\lambda_{2}>\cdots \rightarrow-\infty$ tal que (1.21) tem solução não - nula se e somente se $\lambda=\lambda_{j}, j=1,2, \cdots$. Além disso, se $u_{j}$ é uma solução não - nula de (1.21) quando $\lambda=\lambda_{j}$, então $u_{j}$ tem exatamente $j-1$ zeros no intervalo $(-1,1)$ para cada $j=1,2, \cdots$.

Demonstração. Fixemos $j \geq 1$ e denotemos por $M=\sup _{x \in \Omega} p(x)>0$ e $m=\inf _{x \in \Omega} p(x)>0$. Agora, escolhemos $\bar{\lambda} \in \mathbb{R}$ de forma que a solução $v$ da equação

$$
\left\{\begin{array}{l}
M v^{\prime \prime}+\xi(\bar{\lambda}) v=0, \quad x \in(-1,1) \\
v(-1)=0 \\
v^{\prime}(-1)=1
\end{array}\right.
$$

tenha $j-1$ zeros em $(-1,1]$. Do corolário anterior, segue-se que a solução $u$ de

$$
\left\{\begin{array}{l}
\left(p(x) u^{\prime}\right)^{\prime}+c(x, \lambda) u=0, \quad x \in(-1,0) \cup(0,1) \\
u(0-)=u(0+) \\
p(0-) u^{\prime}(0-)=p(0+) u^{\prime}(0+) \\
u(-1)=0, \quad u^{\prime}(-1)=1
\end{array}\right.
$$

com $\lambda=\bar{\lambda}$ tem pelo menos $j-1$ zeros em $(-1,1]$. Suponha que $u$ tenha $k_{j}-1$ zeros em $(-1,1]$, então $k_{j} \geq j$. Quando $\lambda$ decresce, a partir de $\bar{\lambda}$, a função $\xi(\lambda)$ cresce e portanto, a solução $u$ de (1.23) oscila mais rapidamente, isto é, $u$ vai adquirindo mais zeros. Digamos que para um certo valor $\lambda=\mu_{j}$ a solução $u$ adquire um zero adicional. Seja $z$ este zero adicional. A condição $u(-1)=0$ impede que este zero apareça no ponto $x=-1$. Como $u \not \equiv 0$ não podemos ter $u(z)=0=u_{x}(z)$ e, portanto, $z \notin(-1,0) \cup(0,1)$. Também, $z \neq 0$, pois caso contrário, deveríamos ter $u_{x}\left(0_{-}\right)<0$ e $u_{x}(0+)>0$ ou $u_{x}\left(0_{-}\right)>0$ e $u_{x}(0+)<0$, ou seja, $u$ não seria solução de (1.23). Logo, o zero adicional aparece em $x=1$ e se move, com $\lambda$ decrescendo, na direção de $x=-1$. Para um outro valor $\lambda=\mu_{j+1}$, um outro zero aparece e assim sucessivamente. Portanto, existe uma sequência de números $\mu_{j}, \mu_{j+1}, \mu_{j+2}, \cdots$, com a seguinte propriedade: quando $\mu_{j+1}<\lambda<\mu_{j}$ a solução $u$ da equação $(1.23)$ possui $k_{j}$ zeros em $(-1,1)$ e $u(1) \neq 0$. Para $\lambda=\mu_{j+1}$ a solução da equação $(1.23)$ ainda possui $k_{j}$ zeros em $(-1,1)$ porém satisfaz $u(1)=0$, ou seja, temos uma solução para o problema (1.21).

Observe que a sequência $\left\{\mu_{j}\right\}$ diverge para $-\infty$, pois caso ela se acumulasse em algum ponto $-\infty<\bar{\mu}$ obteríamos uma contradição com o corolário anterior. Agora, 
renomeamos a sequência da seguinte forma: $\lambda_{k_{j}}=\mu_{j}$; assim a solução de (1.21) para $\lambda=\lambda_{k_{j}}$ possui $k_{j}-1$ zeros em $(-1,1)$.

Para finalizar, observemos que, para $\lambda$ suficientemente grande, a solução de (1.23) não possui zeros em $(-1,1]$. Para ver isto basta comparar, em vista do corolário anterior, o número de zeros da solução do problema (1.23) com a solução $w$ do problema

$$
\left\{\begin{array}{l}
m w^{\prime \prime}+\eta(\lambda) w=0, \quad x \in(-1,1) \\
w(-1)=0 \\
w^{\prime}(-1)=1
\end{array}\right.
$$

lembrando que $\eta(\lambda)=\sup _{x \in \Omega} c(x, \lambda) \longrightarrow-\infty$, quando $\lambda \rightarrow+\infty$.

Deste teorema, segue que todos os autovalores do operador $\tilde{A}-\tilde{N}^{\prime}(\varphi)$ são simples. Por outro lado, $\tilde{A}-\tilde{N}^{\prime}(\varphi)$ é autoadjunto, o que implica que seus autovalores são todos reais. A seguir, enunciamos o Corolário 3.5 de [9], que nos permite concluir que o conjunto $\omega$-limite de qualquer ponto é constituído de um único ponto de equilíbrio.

Corolário 1.2 Seja $A$ um operador setorial e $h$ uma função de classe $C^{1}$. Se a equação $u_{t}+A u=h(u)$ é um sistema gradiente com órbitas limitadas precompactas e, para qualquer ponto de equilíbrio $\varphi$, o núcleo do operador $A-h^{\prime}(\varphi)$ tem dimensão menor ou igual a um, e o $\sigma\left(A-h^{\prime}(\varphi)\right) \backslash\{0\}$, é disjunto do eixo imaginário, então o conjunto $\omega$-limite de qualquer ponto é um único ponto de equilibrio.

\subsection{Comportamento assintótico das soluções de equações lineares}

Os resultados que enunciamos a seguir foram obtidos por Henry em [12], onde se encontram as demonstrações. São resultados de Análise Funcional e tratam do comportamento assintótico de soluções especiais de equações lineares de diferença em espaços de Banach cujos corolários têm aplicações no estudo das equações diferenciais.

Teorema 1.5 Sejam $X$ um espaço de Banach e $A_{n}, B_{n}: X \rightarrow X$ operadores lineares 
contínuos, para $n=0,1,2, \cdots[$ ou $n=0,-1,-2, \cdots]$ com

$$
\begin{gathered}
\sum_{n=0}^{+\infty}\left\|A_{n+1}-A_{n}\right\|<\infty, \quad \sum_{n=0}^{+\infty}\left\|B_{n}\right\|<\infty \\
{\left[\text { ou } \sum_{-\infty}^{-1}\left\|A_{n+1}-A_{n}\right\|<\infty, \quad \sum_{-\infty}^{0}\left\|B_{n}\right\|<\infty\right]}
\end{gathered}
$$

Suponha que

$$
A=\lim _{n \rightarrow+\infty} A_{n} \quad\left[\text { ou } \lim _{n \rightarrow-\infty} A_{n}\right]
$$

tem um autovalor simples $\mu \neq 0$ e o restante do espectro de $A$ seja disjunto da circunferência de centro em 0 e raio $|\mu|$. Para $|n|$ suficientemente grande, existe um único autovalor simples $\mu_{n}$ de $A_{n}$ próximo de $\mu$; para outros valores de $n$, seja $\mu_{n}=1$. Seja $v$ o autovetor normalizado de $A, A v=\mu v \neq 0$. Então, existem $n_{0} \in \mathbb{N}$ e uma sequência $\left\{x_{n}\right\}_{n \geq n_{0}}$ em $X\left[\right.$ ou $\left.\left\{x_{n}\right\}_{n \leq-n_{0}}\right]$ tal que

$$
x_{n+1}=\left(A_{n}+B_{n}\right) x_{n} \text { para todo } n \geq n_{0}\left[\text { ou } n \leq-n_{0}\right]
$$

$e$

$$
\begin{gathered}
x_{n}=\prod_{0 \leq k \leq n} \mu_{k}(v+o(1)) \quad \text { quando } n \rightarrow+\infty \\
{\left[\text { ou } x_{n}=\prod_{n \leq k \leq 0} \mu_{k}^{-1}(v+o(1)) \quad \text { quando } n \rightarrow-\infty\right] .}
\end{gathered}
$$

Como consequência obtemos a seguinte aplicação a semigrupos.

Corolário 1.3 Suponhamos que $L(t)$ é o gerador de um $C^{0}$-semigrupo $\left\{e^{s L(t)}: s \geq 0\right\} \subset$ $\mathcal{L}(X)$, para cada $t \geq 0$ [ou $t \leq 0]$. Para cada inteiro $n$, seja $A_{n}=e^{L(n)}$ e suponha que $\left\|e^{s L(n)}\right\|$ seja limitada para $n \geq 0$ [ou $\left.n \leq 0\right]$, uniformemente para $0 \leq s \leq 1$. Ainda, suponhamos que $\left\{A_{n}\right\}_{n \geq 0}\left[\right.$ ou $\left.\left\{A_{n}\right\}_{n \leq 0}\right]$ satisfaça as hipóteses do teorema anterior e seja $\left\{B_{n}\right\}_{n \geq 0}\left[\right.$ ou $\left.\left\{B_{n}\right\}_{n \leq 0}\right]$ uma familia arbitrária de operadores lineares contínuos de $X$ tais que $\sum_{n=0}^{+\infty}\left\|B_{n}\right\|<\infty\left[\right.$ ou $\left.\sum_{-\infty}^{0}\left\|B_{n}\right\|<\infty\right]$. Seja $\left\{x_{n}\right\}_{n \geq n_{0}}\left[\right.$ ou $\left.\left\{x_{n}\right\}_{n \leq-n_{0}}\right]$ a sequência obtida do teorema anterior. Agora, seja $x:\left[n_{0},+\infty\right) \rightarrow X\left[\right.$ ou $\left.x:\left(-\infty,-n_{0}\right] \rightarrow X\right]$ uma função que satisfaça:

(i) $x(n)=x_{n}$, para todo $n \geq n_{0}\left[\right.$ ou $\left.n \leq-n_{0}\right]$; 
(ii) $\left\|x(t+s)-e^{s L(t)} x(t)\right\|=o(\|x(t)\|)$ quando $t \rightarrow+\infty[$ ou $t \rightarrow-\infty]$, uniformemente para $0 \leq s \leq 1$.

Além disso, suponha que $L(t)$ tenha um autovalor $\lambda(t)$ (para $|t|$ grande) com $e^{\lambda(t)} \rightarrow \mu$ quando $t \rightarrow+\infty$ [ou $t \rightarrow-\infty]$ com a correspondente autofunção $v(t) \rightarrow v$, e suponha que

$$
\int^{+\infty}\left|\lambda^{\prime}(t)\right| d t<\infty\left[\text { ou } \int_{-\infty}\left|\lambda^{\prime}(t)\right| d t<\infty\right]
$$

Então, existe uma constante $c \neq 0$ tal que

$$
x(t)=c \exp \left(\int_{t_{0}}^{t} \lambda(r) d r\right)[v+o(1)] \text { quando } t \rightarrow+\infty[\text { ou } t \rightarrow-\infty] .
$$

Teorema 1.6 Sejam $X$ um espaço de Banach e $A: X \rightarrow X$ um operador linear contínuo cujo espectro é disjunto do anel $\{z \in \mathbb{C}: a \leq|z| \leq b\}$, onde $0<a \leq b$. Sejam $X=X_{1} \oplus X_{2}, A=A_{1} \oplus A_{2}$, de modo que $A_{1}=\left.A\right|_{X_{1}}$ tem espectro em $\{z:|z|>b\} e$ $A_{2}=\left.A\right|_{X_{2}}$ tem espectro em $\{z:|z|<a\}$. Seja $\left\{x_{n}\right\}_{n \geq n_{0}}$ uma sequência em $X$ tal que

$$
\left\|x_{n+1}-x_{n}\right\|=o\left(\left\|x_{n}\right\|\right) \quad \text { quando } n \rightarrow+\infty
$$

e $x_{n} \neq 0$, para todo $n$. Se $x_{n}^{j}, j=1,2$, é a componente de $x_{n}$ em $X_{j}$ e c satisfaz $a \leq c \leq b$, então ou

$$
\left\|x_{n}^{2}\right\| /\left\|x_{n}^{1}\right\| \rightarrow 0 \text { e }\left\|x_{n}\right\| / c^{n} \rightarrow+\infty \text { quando } n \rightarrow+\infty
$$

ou

$$
\left\|x_{n}^{2}\right\| /\left\|x_{n}^{1}\right\| \rightarrow+\infty \text { e }\left\|x_{n}\right\| / c^{n} \rightarrow 0 \text { quando } n \rightarrow+\infty
$$

Seja $\left\{x_{n}\right\}_{n \leq-n_{0}}$ uma sequência em $X$ tal que

$$
\left\|x_{n+1}-x_{n}\right\|=o\left(\left\|x_{n}\right\|\right) \quad \text { quando } n \rightarrow-\infty
$$

e $x_{n} \neq 0$, para todo $n$. Se $x_{n}^{j}, j=1,2$, e c são como acima, então ou

$$
\left\|x_{n}^{2}\right\| /\left\|x_{n}^{1}\right\| \rightarrow 0 \text { e }\left\|x_{n}\right\| / c^{n} \rightarrow 0 \text { quando } n \rightarrow-\infty
$$

ou

$$
\left\|x_{n}^{2}\right\| /\left\|x_{n}^{1}\right\| \rightarrow+\infty \text { e }\left\|x_{n}\right\| / c^{n} \rightarrow+\infty \text { quando } n \rightarrow-\infty \text {. }
$$


Corolário 1.4 Suponha que $\left\{e^{t L} ; t \geq 0\right\}$ é um $C^{0}$-semigrupo em $X$ tal que o espectro de $e^{L}$ não intersecta o anel $\left\{z: e^{\alpha} \leq|z| \leq e^{\beta}\right\}$ onde $\alpha<\beta$. Sejam $X=X_{1} \oplus X_{2}, L=$ $L_{1} \oplus L_{2}$ a correspondente decomposição, de modo que $e^{t L_{1}}$ tem espectro em $\left\{z:|z|>e^{\beta t}\right\}$ $e e^{t L_{2}}$ tem espectro em $\left\{z:|z|<e^{\alpha t}\right\}$ quando $t>0$.

Suponha que $x(t) \in X$ para todo $t \geq t_{0}, x(t) \neq 0 e$

$$
\left\|x(t+s)-e^{s L} x(t)\right\|=o(\|x(t)\|)
$$

quando $t \rightarrow+\infty$, uniformemente para $0 \leq s \leq 1$. Então, para qualquer $\gamma$ no intervalo $\alpha \leq \gamma \leq \beta$, temos: ou

$$
\left\|x^{2}(t)\right\| /\left\|x^{1}(t)\right\| \rightarrow 0 \text { e }\|x(t)\| e^{-\gamma t} \rightarrow 0 \text { quando } t \rightarrow+\infty
$$

ou

$$
\left\|x^{2}(t)\right\| /\left\|x^{1}(t)\right\| \rightarrow+\infty \text { e }\|x(t)\| e^{-\gamma t} \rightarrow+\infty \text { quando } t \rightarrow+\infty .
$$

Agora, suponha que $x(t) \in X$ para todo $t \leq-t_{0}, x(t) \neq 0$ e

$$
\left\|x(t+s)-e^{s L} x(t)\right\|=o(\|x(t)\|)
$$

quando $t \rightarrow-\infty$, uniformemente para $0 \leq s \leq 1$. Então para qualquer $\gamma$ no intervalo $\alpha \leq \gamma \leq \beta$, temos: ou

$$
\left\|x^{2}(t)\right\| /\left\|x^{1}(t)\right\| \rightarrow 0 \text { e }\|x(t)\| e^{-\gamma t} \rightarrow+\infty \text { quando } t \rightarrow-\infty
$$

ou

$$
\left\|x^{2}(t)\right\| /\left\|x^{1}(t)\right\| \rightarrow+\infty \text { e }\|x(t)\| e^{-\gamma t} \rightarrow 0 \text { quando } t \rightarrow-\infty
$$

No decorrer do trabalho utilizaremos o seguinte resultado de comparação de semigrupos.

Lema 1.4 Seja $A$ o gerador de um $C^{0}$-semigrupo satisfazendo $\left\|e^{t A}\right\|_{\mathcal{L}(X)} \leq N e^{\omega t}$. Seja $B \in \mathcal{L}(X)$. Então,

$$
\left\|e^{t(A+B)}-e^{t A}\right\|_{\mathcal{L}(X)} \leq N e^{\omega t}\left(e^{t N\|B\|_{\mathcal{L}(X)}}-1\right)
$$

Aplicaremos os resultados acima enunciados para estudarmos o comportamento assintótico das soluções das equações lineares não - autônomas. 
Teorema 1.7 Sejam $a(x), b(x), c(x, t)$ e $d(x, t)$ funções reais. Suponha que $a, b \in$ $C^{1}([0,1],(0, \infty)), c(x, t)$ e $d(x, t)$ têm a primeira derivada com relação a $t$ contínua $e$ limitada em $-\infty<t<+\infty, 0 \leq x \leq 1$, e

$$
\int_{-\infty}^{+\infty} \sup _{x \in[0,1]}\left\{\left|c_{t}(x, t)\right|,\left|d_{t}(x, t)\right|\right\} d t<\infty .
$$

Seja $D(L(t))=D(A)=\left\{(u, v) \in H^{2}(0,1) \times H^{2}(0,1): a(0) u_{x}(0)+b(0) v_{x}(0)=\right.$ $0, u(0)=v(0), u(1)=v(1)=0\}$ e defina $L(t): D(L(t)) \subset L^{2}(0,1) \times L^{2}(0,1) \longrightarrow$ $L^{2}(0,1) \times L^{2}(0,1)$ por

$$
[L(t)(u, v)](x)=\left(\left(a(x) u_{x}(x)\right)_{x}+c(x, t) u(x),\left(b(x) v_{x}(x)\right)_{x}+d(x, t) v(x)\right),
$$

para $x \in(0,1)$. Defina também $L_{ \pm}: D\left(L_{ \pm}\right) \subset L^{2}(0,1) \times L^{2}(0,1) \longrightarrow L^{2}(0,1) \times L^{2}(0,1)$ por

$$
\left[L_{ \pm}(u, v)\right](x)=\left(\left(a(x) u_{x}(x)\right)_{x}+c_{ \pm}(x) u(x),\left(b(x) v_{x}(x)\right)_{x}+d_{ \pm}(x) v(x)\right),
$$

para $x \in(0,1)$, sendo $D\left(L_{ \pm}\right)=D(L(t))=D(A)$ e $c_{ \pm}(x)=\lim _{t \rightarrow \pm \infty} c(x, t)$ e $d_{ \pm}(x)=$ $\lim _{t \rightarrow \pm \infty} d(x, t)$.

Os operadores $-L(t)$, para cada $t$ fixo, e $-L_{ \pm}$são setoriais. Seja $\lambda_{j}(t)$ o $j$-ésimo autovalor de $L(t)$, lembrando que $\lambda_{1}(t)>\lambda_{2}(t)>\cdots>\lambda_{j}(t) \rightarrow-\infty$ quando $j \rightarrow+\infty$, e seja $\lambda_{j}^{ \pm}$o j-ésimo autovalor de $L_{ \pm}$. Note que $\lambda_{j}(t) \rightarrow \lambda_{j}^{ \pm}$quando $t \rightarrow \pm \infty$. Seja $\psi_{j}^{ \pm}=\left(\psi_{1 j}^{ \pm}, \psi_{2 j}^{ \pm}\right)$a j-ésima autofunção de $L_{ \pm}$.

Então existem soluções clássicas $w_{j}^{ \pm}(\cdot, t)=\left(u_{j}^{ \pm}(\cdot, t), v_{j}^{ \pm}(\cdot, t)\right)$ do problema

$$
\left\{\begin{array}{l}
u_{t}=\left(a(x) u_{x}\right)_{x}+c(x, t) u, x \in(0,1) \\
v_{t}=\left(b(x) v_{x}\right)_{x}+d(x, t) v, \quad x \in(0,1) \\
u(0, t)=v(0, t) \\
a(0) u_{x}(0, t)+b(0) v_{x}(0, t)=0 \\
u(1, t)=v(1, t)=0
\end{array}\right.
$$

com $w_{j}^{-}$definida em $-\infty<t<+\infty$ e $w_{j}^{+}$definida para $t$ maior do que algum $t_{j}$, tais $q u e$

$$
\begin{gathered}
w_{j}^{+}(t)=\exp \left(\int_{0}^{t} \lambda_{j}(s) d s\right)\left[\psi_{j}^{+}+o(1)\right] \text { quando } t \rightarrow+\infty \\
w_{j}^{-}(t)=\exp \left(-\int_{t}^{0} \lambda_{j}(s) d s\right)\left[\psi_{j}^{-}+o(1)\right] \text { quando } t \rightarrow-\infty
\end{gathered}
$$

com as convergências na norma de $C^{1}[0,1] \times C^{1}[0,1]$. 
Demonstração. Consideraremos o caso $t \rightarrow+\infty$; o caso $t \rightarrow-\infty$ pode ser feito de maneira análoga.

Reescrevendo o sistema (1.25) como uma equação de evolução temos

$$
w_{t}=L(t) w
$$

para $w=(u, v)$.

Seja $\{T(t, s), t \geq s\}$ o operador de evolução da equação (1.26), no sentido do Teorema 7.1.3 em Henry [11], de modo que $w(t)=T(t, s) w(s)$ quando $w$ resolve a equação (1.26) no intervalo $[s, t]$.

Considerando $-L_{+}$como operador setorial principal em $X=L^{2}(0,1) \times L^{2}(0,1)$, de modo que as normas $\|\cdot\|_{\alpha}$ são definidas por

$$
X^{\alpha}=D\left(\left(a-L_{+}\right)^{\alpha}\right), \quad\|(u, v)\|_{\alpha}=\left\|\left(a-L_{+}\right)^{\alpha}(u, v)\right\|_{X}, \quad \alpha \geq 0,
$$

onde $a>0$ é escolhida suficientemente grande de modo que $\operatorname{Re} \sigma\left(a-L_{+}\right)>0$.

Segue do Teorema 1.6.1 em Henry [11], que $X^{\alpha} \hookrightarrow C^{1}[0,1] \times C^{1}[0,1]$ para todo $3 / 4<\alpha \leq 1$. Além disso, não é difícil mostrar que a $\|\cdot\|_{1 / 2}$ é equivalente à norma de $H=\left\{(u, v) \in H^{1}(0,1) \times H^{1}(0,1): u(0)=v(0), u(1)=v(1)=0\right\}$, lembrando que

$$
\|(u, v)\|_{H}^{2}=\left\|u^{\prime}\right\|_{L^{2}}^{2}+\left\|v^{\prime}\right\|_{L^{2}}^{2} .
$$

Do Teorema 7.1.3 em Henry [11], segue que

$$
\|T(t, s)(\varphi, \psi)\|_{X} \leq C\|(\varphi, \psi)\|_{X}
$$

$\mathrm{e}$

$$
\|T(t, s)(\varphi, \psi)\|_{H} \leq 2 C(t-s)^{-1 / 2}\|(\varphi, \psi)\|_{X}
$$

para $0<t-s \leq 1$, sendo $C$ uma constante que depende somente de $-L_{+}$e de $\sup \{|c(x, t)|,|d(x, t)|\}$.

$0 \leq x \leq 1$

Fixe $\tau$ e considere $t$ de forma que $0<t-\tau \leq 1$. Escrevendo (1.26) como

$$
w_{t}=L(\tau) w+[L(t)-L(\tau)] w
$$

e usando a fórmula da variação das constantes, obtemos

$$
\begin{aligned}
T(t, \tau) w & =e^{L(\tau)(t-\tau)} w+\int_{\tau}^{t} e^{L(\tau)(t-s)}(L(s)-L(\tau)) T(s, \tau) w d s \\
& =e^{L(\tau)(t-\tau)} w+\int_{\tau}^{t} e^{L(\tau)(t-s)}\left(\int_{\tau}^{s} L^{\prime}(r) d r\right) T(s, \tau) w d s
\end{aligned}
$$


onde

$$
\left[L^{\prime}(r)(u, v)\right](x)=\left(c_{r}(x, r) u(x), d_{r}(x, r) v(x)\right), \quad 0 \leq x \leq 1 .
$$

Se $0 \leq \alpha<1$, então

$$
\begin{aligned}
\| T(t, \tau) w & -e^{L(\tau)(t-\tau)} w\left\|_{\alpha}=\right\| \int_{\tau}^{t}\left(a-L_{+}\right)^{\alpha} e^{L(\tau)(t-s)}\left(\int_{\tau}^{s} L^{\prime}(r) d r\right) T(s, \tau) w d s \|_{X} \\
& \leq \int_{\tau}^{t}\left\|\left(a-L_{+}\right)^{\alpha} e^{L(\tau)(t-s)}\right\|_{\mathcal{L}(X)}\left\|\int_{\tau}^{s} L^{\prime}(r) d r\right\|_{\mathcal{L}(H, X)}\|T(s, \tau) w\|_{H} d s .
\end{aligned}
$$

Como $0<t-s \leq 1$, obtemos

$$
\begin{aligned}
\left\|\left(a-L_{+}\right)^{\alpha} e^{L(\tau)(t-s)}\right\|_{\mathcal{L}(X)} & =\left\|\left(a-L_{+}\right)^{\alpha} e^{-\left(a-L_{+}\right)(t-s)} e^{\left(a-L_{+}+L(\tau)\right)(t-s)}\right\|_{\mathcal{L}(X)} \\
& \leq\left\|\left(a-L_{+}\right)^{\alpha} e^{-\left(a-L_{+}\right)(t-s)}\right\|_{\mathcal{L}(X)}\left\|e^{\left(a-L_{+}+L(\tau)\right)(t-s)}\right\|_{\mathcal{L}(X)} \\
& \leq C_{\alpha}(t-s)^{-\alpha} .
\end{aligned}
$$

Também,

$$
\begin{aligned}
\left\|L^{\prime}(r)(u, v)\right\|_{X}^{2} & =\int_{0}^{1}\left(\mid\left(\left.c_{r}(x, r) u(x)\right|^{2}+\left|d_{r}(x, r) v(x)\right|^{2}\right) d x\right. \\
& \leq\left[\sup _{0 \leq x \leq 1}\left\{\left|c_{r}(x, r)\right|,\left|d_{r}(x, r)\right|\right\}\right]^{2} \int_{0}^{1}\left(|u(x)|^{2}+|v(x)|^{2}\right) d x \\
& \leq \gamma(r)^{2}\|(u, v)\|_{H}^{2},
\end{aligned}
$$

onde $\gamma(r):=\sup _{0 \leq x \leq 1}\left\{\left|c_{r}(x, r)\right|,\left|d_{r}(x, r)\right|\right\}$. Assim,

$$
\left\|\int_{\tau}^{s} L^{\prime}(r) d r\right\|_{\mathcal{L}(H, X)} \leq \int_{\tau}^{t} \gamma(r) d r
$$

Das estimativas acima, juntamente com (1.27) e (1.28), segue que

$$
\begin{aligned}
\left\|T(t, \tau) w-e^{L(\tau)(t-\tau)} w\right\|_{\alpha} & \leq C C_{\alpha}\left(\int_{\tau}^{t} \gamma(r) d r\right)\left(\int_{\tau}^{t}(t-s)^{-\alpha}(s-\tau)^{-1 / 2} d s\right)\|w\|_{X} \\
& \leq C C_{\alpha} B(1 / 2,1-\alpha)\left(\int_{\tau}^{t} \gamma(r) d r\right)(t-\tau)^{1 / 2-\alpha}\|w\|_{X}
\end{aligned}
$$

onde $B(\cdot, \cdot)$ é a função beta. Se $\bar{C}_{\alpha}:=C C_{\alpha} B(1 / 2,1-\alpha)$ então

$$
\left\|T(t, \tau) w-e^{L(\tau)(t-\tau)} w\right\|_{\alpha} \leq \bar{C}_{\alpha}\left(\int_{\tau}^{t} \gamma(r) d r\right)(t-\tau)^{1 / 2-\alpha}\|w\|_{X} .
$$

Considerando os operadores lineares limitados $A_{n}:=e^{L(n)}$ e $B_{n}:=T(n+1, n)-A_{n}$, onde $n \in \mathbb{Z}^{+}$, usando (1.29) obtemos

$$
\left\|B_{n}\right\|_{\mathcal{L}(X)}=\sup _{\|w\|_{X} \leq 1}\left\|T(n+1, n) w-A_{n} w\right\|_{X} \leq \bar{C}_{\alpha}\left(\int_{n}^{n+1} \gamma(r) d r\right) .
$$


Portanto,

$$
\sum_{n=0}^{+\infty}\left\|B_{n}\right\|_{\mathcal{L}(X)} \leq \bar{C}_{\alpha} \sum_{n=0}^{+\infty}\left(\int_{n}^{n+1} \gamma(r) d r\right)=\bar{C}_{\alpha} \int_{0}^{+\infty} \gamma(r) d r<\infty .
$$

Como

$$
\begin{aligned}
A_{n+1}-A_{n} & =\int_{0}^{1} \frac{d}{d s}\left[e^{s L(n+1)} e^{(1-s) L(n)}\right] d s \\
& =\int_{0}^{1} e^{(1-s) L(n)}[L(n+1)-L(n)] e^{s L(n+1)} d s \\
& =\int_{0}^{1} e^{(1-s) L(n)}\left(\int_{n}^{n+1} L^{\prime}(r) d r\right) e^{s L(n+1)} d s
\end{aligned}
$$

obtemos

$$
\begin{aligned}
\left\|A_{n+1}-A_{n}\right\|_{\mathcal{L}(X)} & \leq \int_{0}^{1}\left\|e^{(1-s) L(n)}\right\|\left\|\int_{n}^{n+1} L^{\prime}(r) d r\right\|\left\|e^{s L(n+1)}\right\| d s \\
& \leq C^{\prime} \int_{n}^{n+1} \gamma(r) d r
\end{aligned}
$$

o que implica

$$
\sum_{n=0}^{+\infty}\left\|A_{n+1}-A_{n}\right\|_{\mathcal{L}(X)} \leq C^{\prime} \int_{0}^{\infty} \gamma(r) d r<\infty
$$

Tomando $e^{L_{+}}=\lim _{n \rightarrow+\infty} A_{n}$, as condições Teorema 1.5 estão satisfeitas. Se $\left\{w_{n}: n \geq\right.$ $\left.n_{0}\right\}$ é a sequência proveniente do Teorema 1.5 , então $\frac{w_{n}}{\left\|w_{n}\right\|} \rightarrow \psi_{j}^{+}$quando $n \rightarrow+\infty$ e $w_{n+1}=\left(A_{n}+B_{n}\right) w_{n}:=T(n+1, n) w_{n}$, para todo $n \geq n_{0}$.

Defina $w(t):=T\left(t, n_{0}\right) w_{n_{0}}$, para $t \geq n_{0}$. Como $w\left(n_{0}\right)=w_{n_{0}}$ e $w(k+1)=T(k+$ $\left.1, n_{0}\right) w_{n_{0}}=T(k+1, k) T\left(k, n_{0}\right) w_{n_{0}}$, para todo $k \geq n_{0}$, segue-se, por indução, que $w(n)=$ $w_{n}$ para todo $n \geq n_{0}$.

Considerando $0 \leq s \leq 1, t \geq n_{0}$ e usando (1.29), com $\alpha=0$, obtemos

$$
\begin{aligned}
\left\|w(t+s)-e^{s L(t)} w(t)\right\|_{X} & =\left\|T\left(t+s, n_{0}\right) w_{n_{0}}-e^{s L(t)} w(t)\right\|_{X} \\
& =\left\|T(t+s, t) T\left(t, n_{0}\right) w_{n_{0}}-e^{s L(t)} w(t)\right\|_{X} \\
& =\left\|T(t+s, t) w(t)-e^{s L(t)} w(t)\right\|_{X} \\
& \leq \bar{C}_{0}\left(\int_{t}^{t+s} \gamma(r) d r\right)\|w(t)\|_{X} .
\end{aligned}
$$

Isto implica que

$$
\frac{\left\|w(t+s)-e^{s L(t)} w(t)\right\|_{X}}{\|w(t)\|_{X}} \leq \bar{C}_{0} \int_{t}^{t+s} \gamma(r) d r \longrightarrow 0
$$


quando $t \rightarrow+\infty$.

Portanto, $\left\|w(t+s)-e^{s L(t)} w(t)\right\|_{X}=o\left(\|w(t)\|_{X}\right)$ quando $t \rightarrow+\infty$, uniformemente para $0 \leq s \leq 1$.

Segue do Corolário 1.3 que existe uma constante $\hat{C} \neq 0$ tal que

$$
w(t)=\hat{C} \exp \left(\int_{n_{0}}^{t} \lambda_{j}(s) d s\right)\left[\psi_{j}^{+}+o(1)\right] \text { quando } t \rightarrow+\infty,
$$

isto é,

$$
w(t)=C \exp \left(\int_{0}^{t} \lambda_{j}(s) d s\right)\left[\psi_{j}^{+}+o(1)\right] \text { quando } t \rightarrow+\infty
$$

onde $C:=\hat{C} \exp \left(-\int_{0}^{n_{0}} \lambda_{j}(s) d s\right)$ e a convergência é em $X=L^{2}(0,1) \times L^{2}(0,1)$.

Verifiquemos que a convergência ocorre na norma $\|\cdot\|_{\alpha}$ para qualquer $\alpha<1$, o que assegurará a convergência $C^{1}([0,1]) \times C^{1}([0,1])$. Para ver isto, seja

$$
z(t)=\frac{1}{C} \exp \left(-\int_{0}^{t} \lambda_{j}(s) d s\right) w(t)
$$

então $z(t) \rightarrow \psi_{j}^{+}$em $X$ quando $t \rightarrow+\infty$.

Seja $\psi_{j}(t)$ autofunção de $L(t)$ tal que $\psi_{j}(t) \rightarrow \psi_{j}^{+}$quando $t \rightarrow+\infty$ em $H$. Então $z(t)-\psi_{j}(t) \rightarrow 0$ em $X$ quando $t \rightarrow+\infty$.

Como $w(t+1)=T(t+1, t) w(t)$, segue que

$$
\begin{gathered}
\left\|\exp \left(\int_{t}^{t+1} \lambda_{j}(s) d s\right) z(t+1)-e^{L(t)} z(t)\right\|_{\alpha}=\left\|\frac{1}{C} \exp \left(-\int_{0}^{t} \lambda_{j}(s) d s\right) w(t+1)-e^{L(t)} z(t)\right\|_{\alpha}= \\
=\left\|\frac{1}{C} \exp \left(-\int_{0}^{t} \lambda_{j}(s) d s\right) T(t+1, t) w(t)-e^{L(t)} z(t)\right\|_{\alpha}=\left\|T(t+1, t) z(t)-e^{L(t)} z(t)\right\|_{\alpha} \leq \\
\leq \bar{C}_{\alpha}\left(\int_{t}^{t+1} \gamma(r) d r\right)\|z(t)\|_{X} \longrightarrow 0 \text { quando } t \rightarrow+\infty .
\end{gathered}
$$

Portanto,

$$
\begin{aligned}
& \exp \left(\int_{t}^{t+1} \lambda_{j}(s) d s\right)\left\|z(t+1)-\psi_{j}(t)\right\|_{\alpha} \leq\left\|\exp \left(\int_{t}^{t+1} \lambda_{j}(s) d s\right) z(t+1)-e^{L(t)} z(t)\right\|_{\alpha}+ \\
& \quad+\left\|e^{L(t)} z(t)-e^{L(t)} \psi_{j}(t)\right\|_{\alpha}+\left\|e^{L(t)} \psi_{j}(t)-\exp \left(\int_{t}^{t+1} \lambda_{j}(s) d s\right) \psi_{j}(t)\right\|_{\alpha}= \\
& =o(1)+\left\|e^{L(t)} z(t)-e^{L(t)} \psi_{j}(t)\right\|_{\alpha}+\left\|e^{L(t)} \psi_{j}(t)-\exp \left(\int_{t}^{t+1} \lambda_{j}(s) d s\right) \psi_{j}(t)\right\|_{\alpha} \cdot(1.30)
\end{aligned}
$$


Cormo $\lambda_{j}(t) \rightarrow \lambda_{j}^{+}$quando $t \rightarrow+\infty$, segue que $\int_{t}^{t+1} \lambda_{j}(s) d s \rightarrow \lambda_{j}^{+}$quando $t \rightarrow+\infty$, com isto obtemos

$$
\begin{aligned}
\left\|e^{L(t)} \psi_{j}(t)-\exp \left(\int_{t}^{t+1} \lambda_{j}(s) d s\right) \psi_{j}(t)\right\|_{\alpha} & =\left\|e^{\lambda_{j}(t)} \psi_{j}(t)-\exp \left(\int_{t}^{t+1} \lambda_{j}(s) d s\right) \psi_{j}(t)\right\|_{\alpha} \\
& \leq\left|e^{\lambda_{j}(t)}-\exp \left(\int_{t}^{t+1} \lambda_{j}(s) d s\right)\right|\left\|\psi_{j}(t)\right\|_{\alpha} \rightarrow 0
\end{aligned}
$$

quando $t \rightarrow+\infty$. Por sua vez,

$$
\begin{aligned}
\left\|e^{L(t)}\left(z(t)-\psi_{j}(t)\right)\right\|_{\alpha} & =\left\|\left(a-L_{+}\right)^{\alpha} e^{L(t)}\left(z(t)-\psi_{j}(t)\right)\right\|_{X} \\
& =\left\|\left(a-L_{+}\right)^{\alpha} e^{-\left(a-L_{+}\right)} e^{\left(a-L_{+}+L(t)\right)}\left(z(t)-\psi_{j}(t)\right)\right\|_{X} \\
& \leq C_{\alpha}\left\|e^{\left(a-L_{+}+L(t)\right)}\right\|\left\|z(t)-\psi_{j}(t)\right\|_{X} \longrightarrow 0
\end{aligned}
$$

quando $t \rightarrow+\infty$. Portanto, concluímos de (1.30) que

$$
\left\|z(t+1)-\psi_{j}(t)\right\|_{\alpha} \longrightarrow 0 \text { quando } t \rightarrow+\infty
$$

Como $\left\|\psi_{j}(t)-\psi_{j}^{+}\right\|_{\alpha} \rightarrow 0$ quando $t \rightarrow+\infty$, segue que $\left\|z(t)-\psi_{j}^{+}\right\|_{\alpha} \rightarrow 0$ quando $t \rightarrow+\infty$, o que completa a demonstração.

Corolário 1.5 Suponha que as hipóteses do Teorema 1.7 estejam satisfeitas. Então o problema adjunto

$$
\left\{\begin{array}{l}
\theta_{t}+\left(a(x) \theta_{x}\right)_{x}+c(x, t) \theta=0, \quad x \in(0,1) \\
\vartheta_{t}+\left(b(x) \vartheta_{x}\right)_{x}+d(x, t) \vartheta=0, \quad x \in(0,1) \\
\theta(0, t)=\vartheta(0, t) \\
a(0) \theta_{x}(0, t)+b(0) \vartheta_{x}(0, t)=0 \\
\theta(1, t)=\vartheta(1, t)=0
\end{array}\right.
$$

tem soluções clássicas $\eta_{j}^{ \pm}=\left(\theta_{j}^{ \pm}, \vartheta_{j}^{ \pm}\right),\left(\eta_{j}^{-}\right.$definida somente para $\left.t<t_{j}^{*}\right)$ tais que

$$
\begin{gathered}
\eta_{j}^{+}(t)=\exp \left(-\int_{0}^{t} \lambda_{j}(s) d s\right)\left[\psi_{j}^{+}+o(1)\right] \text { quando } t \rightarrow+\infty \\
\eta_{j}^{-}(t)=\exp \left(\int_{t}^{0} \lambda_{j}(s) d s\right)\left[\psi_{j}^{-}+o(1)\right] \text { quando } t \rightarrow-\infty
\end{gathered}
$$

com as convergências na norma de $C^{1}[0,1] \times C^{1}[0,1]$. Aqui $\psi_{j}^{ \pm}$indica a j-ésima autofunção dos operadores $L_{ \pm}^{*}=L_{ \pm}$. 
Demonstração. Reescrevemos o problema adjunto como $\eta_{t}=-L(t) \eta$, onde $L(t)$ é o operador usado no Teorema 1.7. Fazendo a mudança de variável $t=-\tau$, obtemos um novo problema na variável temporal $\tau$, para o qual podemos aplicar o Teorema 1.7 e a conclusão segue.

Observação 1.1 Se $w=(u, v)$ resolve $(1.25)$ e $\eta=(\theta, \vartheta)$ resolve (1.31) no intervalo $t_{0}<t<t_{1}$, então

$$
t \longmapsto \int_{0}^{1}\{u(x, t) \theta(x, t)+v(x, t) \vartheta(x, t)\} d x
$$

é constante nesse intervalo.

Teorema 1.8 Com as mesmas hipóteses e notações do Teorema 1.7, seja $w(t)=(u(t), v(t))$ uma solução de (1.25) para $t>t_{0}$, não identicamente nula. Suponha que a aplicação

$$
\Phi(t)=\sup _{0 \leq x \leq 1}\left\{\left|c(x, t)-c_{+}(x)\right|,\left|d(x, t)-d_{+}(x)\right|\right\}
$$

esteja em $L^{\infty}(0, \infty) \cap L^{1}(0, \infty)$. Então, existem um inteiro $j \geq 1$ e uma constante $C \neq 0$ tais que

$$
w(t)=\exp \left(\int_{0}^{t} \lambda_{j}(s) d s\right)\left[C \psi_{j}^{+}+o(1)\right] \text { quando } t \rightarrow+\infty,
$$

com convergência na norma de $C^{1}[0,1] \times C^{1}[0,1]$. Na realidade, dada qualquer constante $k$, se definirmos $m$ por $\lambda_{m+1}^{+}<-k \leq \lambda_{m}^{+}$, então existem constantes $C_{1}, C_{2}, \cdots, C_{m}$ tais que

$$
w(t)=\sum_{j=1}^{m} C_{j} w_{j}^{+}(t)+o\left(e^{-k t}\right) \text { quando } t \rightarrow+\infty
$$

Demonstração. Com algumas estimativas simples, é fácil mostrar que $\|w(t)\|_{X}=$ $O\left(e^{\delta t}\right)$ quando $t \rightarrow+\infty$, para alguma constante $\delta$; assim

$$
\mu:=\limsup _{t \rightarrow+\infty}\left(\frac{1}{t} \log \|w(t)\|_{X}\right) \leq \delta<+\infty .
$$

Por outro lado, vamos provar que $-\infty<\mu$ quando $w \not \equiv 0$. Podemos escrever (1.26) como

$$
w_{t}=L_{+} w+\left(L(t)-L_{+}\right) w
$$


Desta forma,

$$
\begin{aligned}
\left\|w_{t}-L_{+} w\right\|_{X}^{2} & =\left\|\left(L(t)-L_{+}\right) w\right\|_{X}^{2} \\
& =\int_{0}^{1}\left[\left|c(x, t)-c_{+}(x)\right|^{2}|u(x)|^{2}+\left|d(x, t)-d_{+}(x)\right|^{2}|v(x)|^{2}\right] d x \\
& \leq\left[\sup _{0 \leq x \leq 1}\left\{\left|c(x, t)-c_{+}(x)\right|,\left|d(x, t)-d_{+}(x)\right|\right\}\right]^{2}\|(u, v)\|_{X}^{2} \\
& =\Phi(t)^{2}\|w\|_{X}^{2} .
\end{aligned}
$$

Como $L_{+}$é simétrico e, para cada $\varphi \in D\left(L_{+}\right)$, a função vetorial $t \mapsto L_{+} \varphi \in X$ é diferenciável e a função $\Phi(t) \in L^{\infty}(0, \infty) \cap L^{1}(0, \infty)$, temos verificadas as hipóteses da Proposição 2.1, Capítulo 2, de Agmon [2], que implica a existência de uma constante $\sigma>0$, dependendo de $w\left(t_{0}\right)$, tal que

$$
\|w(t)\|_{X} \geq e^{-\sigma\left(t-t_{0}\right)}\left\|w\left(t_{0}\right)\right\|_{X}, \quad \text { para todo } t \geq t_{0}+1
$$

donde segue que $\mu \geq-\sigma$.

Indiquemos $\lambda_{0}=+\infty$ e escolhamos $j \geq 1$ tal que $\lambda_{j}^{+} \leq \mu<\lambda_{j-1}^{+}$. Pela estimativa (1.29), para $0 \leq s \leq 1$, temos

$$
\begin{aligned}
\left\|w(t+s)-e^{s L_{+}} w(t)\right\|_{X} & =\left\|T(t+s, t) w(t)-e^{s L_{+}} w(t)\right\|_{X} \\
& \leq\left\|T(t+s, t) w(t)-e^{s L(t)} w(t)\right\|_{X}+\left\|e^{s L(t)} w(t)-e^{s L_{+}} w(t)\right\|_{X} \\
& \leq\left[\bar{C}_{0} \int_{t}^{t+s} \gamma(r) d r+\left\|e^{s L(t)}-e^{s L_{+}}\right\|_{\mathcal{L}(X)}\right]\|w(t)\|_{X} .
\end{aligned}
$$

Como

$$
\left\|\left(L(t)-L_{+}\right) w\right\|_{\mathcal{L}(X)} \leq \Phi(t)\|w\|
$$

usando o Lema 1.4 temos

$$
\left\|e^{s L(t)}-e^{s L_{+}}\right\| \leq N e^{\theta}\left(e^{N \Phi(t)}-1\right) \longrightarrow 0
$$

quando $t \rightarrow+\infty$, uniformemente em $0 \leq s \leq 1$, para constantes $N$ e $\theta$.

Com isto obtemos

$$
\left\|w(t+s)-e^{s L_{+}} w(t)\right\|_{X}=o\left(\|w(t)\|_{X}\right)
$$

quando $t \rightarrow+\infty$, uniformemente para $0 \leq s \leq 1$. 
Escolhendo $\alpha$ e $\beta$ tais que $\lambda_{j+1}^{+}<\alpha<\beta<\lambda_{j}^{+} \leq \mu$, temos $\sigma\left(e^{L_{+}}\right) \cap\left\{z \in \mathbb{C}: e^{\alpha} \leq\right.$ $\left.|z| \leq e^{\beta}\right\}=\emptyset$.

Sejam $X_{1}=\left[\psi_{1}^{+}, \cdots, \psi_{j}^{+}\right]$e $X_{2}=\left[\psi_{j+1}^{+}, \psi_{j+2}^{+}, \cdots\right]$, onde $\left[\psi_{1}^{+}, \cdots, \psi_{j}^{+}\right]$indica o espaço gerado pelas funções $\psi_{1}^{+}, \cdots, \psi_{j}^{+}$. Então $X=X_{1} \oplus X_{2}, L_{+}=L_{+}^{1} \oplus L_{+}^{2}, \sigma\left(e^{t L_{+}^{1}}\right)=\{z \in$ $\left.\sigma\left(e^{t L_{+}}\right):|z|>e^{\beta t}\right\}$ e $\sigma\left(e^{t L_{+}^{2}}\right)=\left\{z \in \sigma\left(e^{t L_{+}}\right):|z|<e^{\alpha t}\right\}$.

Segue do Corolário 1.6, para qualquer $\alpha \leq \gamma \leq \beta$, que

$$
\frac{\left\|w_{2}(t)\right\|_{X}}{\left\|w_{1}(t)\right\|_{X}} \rightarrow 0 \text { e }\|w(t)\|_{X} e^{-\gamma t} \rightarrow 0 \quad \text { quando } t \rightarrow+\infty
$$

ou

$$
\frac{\left\|w_{2}(t)\right\|_{X}}{\left\|w_{1}(t)\right\|_{X}} \rightarrow+\infty \text { e }\|w(t)\|_{X} e^{-\gamma t} \rightarrow+\infty \text { quando } t \rightarrow+\infty .
$$

Como $\alpha<\mu$ e $\mu=\lim \sup _{t \rightarrow+\infty}\left(\frac{1}{t} \log \|w(t)\|_{X}\right)$, então $\|w(t)\|_{X}$ não é $o\left(e^{\alpha t}\right)$ quando $t \rightarrow+\infty$ e desta forma, a condição (1.32) não é satisfeita para $\gamma=\alpha$; logo (1.33) se verifica. Em outras palavras, a componente de $w(t)$ em $\left[\psi_{1}^{+}, \cdots, \psi_{j}^{+}\right]$é suficientemente pequena se comparada com $\|w(t)\|_{X}$ quando $t \rightarrow+\infty$.

Agora, escolhendo $\alpha$ e $\beta$ tais que $\lambda_{j}^{+} \leq \mu<\alpha<\beta<\lambda_{j-1}^{+}$, temos $\sigma\left(e^{L_{+}}\right) \cap\{z \in \mathbb{C}$ : $\left.e^{\alpha} \leq|z| \leq e^{\beta}\right\}=\emptyset$.

Sejam $X_{1}=\left[\psi_{1}^{+}, \cdots, \psi_{j-1}^{+}\right]$e $X_{2}=\left[\psi_{j}^{+}, \psi_{j+1}^{+}, \cdots\right]$, então $X=X_{1} \oplus X_{2}, L_{+}=L_{+}^{1} \oplus L_{+}^{2}$, $\sigma\left(e^{t L_{+}^{1}}\right)=\left\{z \in \sigma\left(e^{t L_{+}}\right):|z|>e^{\beta t}\right\}$ e $\sigma\left(e^{t L_{+}^{2}}\right)=\left\{z \in \sigma\left(e^{t L_{+}}\right):|z|<e^{\alpha t}\right\}$.

Usando novamente o Corolário 1.6, sabemos que se $\alpha \leq \gamma \leq \beta$, então

$$
\frac{\left\|w_{2}(t)\right\|_{X}}{\left\|w_{1}(t)\right\|_{X}} \rightarrow 0 \text { e }\|w(t)\|_{X} e^{-\gamma t} \rightarrow 0 \text { quando } t \rightarrow+\infty
$$

ou

$$
\frac{\left\|w_{2}(t)\right\|_{X}}{\left\|w_{1}(t)\right\|_{X}} \rightarrow+\infty \text { e }\|w(t)\|_{X} e^{-\gamma t} \rightarrow+\infty \text { quando } t \rightarrow+\infty .
$$

Como $\mu<\beta$ e $\mu=\lim \sup _{t \rightarrow+\infty}\left(\frac{1}{t} \log \|w(t)\|_{X}\right)$ segue que $\|w(t)\|_{X}=O\left(e^{\beta t}\right)$ quando $t \rightarrow+\infty$. Assim, a condição (1.35) não se verifica para $\gamma=\beta$; consequentemente a condição (1.34) é válida, em outras palavras, a componente de $w(t)$ em $\left[\psi_{j}^{+}, \psi_{j+1}^{+}, \cdots\right]$ é suficientemente pequena se comparada com $\|w(t)\|_{X}$ quando $t \rightarrow+\infty$.

Sabemos que existem funções reais $\sigma(t)$ e $\alpha_{k}(t)$ tais que

$$
\frac{\left\|w(t)-\sigma(t) \psi_{j}^{+}\right\|_{X}}{\|w(t)\|_{X}} \leq \frac{\left\|\sum_{k=1}^{j-1} \alpha_{k}(t) \psi_{k}^{+}\right\|_{X}}{\|w(t)\|_{X}}+\frac{\left\|\sum_{k=j+1}^{+\infty} \alpha_{k}(t) \psi_{k}^{+}\right\|_{X}}{\|w(t)\|_{X}}
$$


Segue das informações acima que

$$
\frac{\left\|w(t)-\sigma(t) \psi_{j}^{+}\right\|_{X}}{\|w(t)\|_{X}} \rightarrow 0 \text { e } \frac{|\sigma(t)|}{\|w(t)\|_{X}} \rightarrow 1
$$

quando $t \rightarrow+\infty$. Sejam

$$
z(t)=\exp \left(-\int_{0}^{t} \lambda_{j}(s) d s\right) w(t) \text { e } C(t)=\exp \left(-\int_{0}^{t} \lambda_{j}(s) d s\right) \sigma(t)
$$

Então,

$$
\frac{\left\|z(t)-C(t) \psi_{j}^{+}\right\|_{X}}{\|z(t)\|_{X}} \rightarrow 0 \text { e } \frac{|C(t)|}{\|z(t)\|_{X}} \rightarrow 1
$$

quando $t \rightarrow+\infty$. Vamos mostrar que $C(t)$ converge para um limite diferente de zero quando $t \rightarrow+\infty$. Temos

$$
\begin{aligned}
\| z(t+s) \exp \left(\int_{t}^{t+s}\right. & \left.\lambda_{j}(r) d r\right)-e^{s L(t)} z(t) \|_{X}= \\
& =\left\|\exp \left(-\int_{0}^{t} \lambda_{j}(r) d r\right) T(t+s, t) w(t)-e^{s L(t)} z(t)\right\|_{X} \\
& =\left\|T(t+s, t) z(t)-e^{s L(t)} z(t)\right\|_{X} \\
& \leq \bar{C}_{0}\left(\int_{t}^{t+s} \gamma(r) d r\right)\|z(t)\|_{X},
\end{aligned}
$$

para $0 \leq s \leq 1$ e $\int_{0}^{+\infty} \gamma(r) d r<\infty$.

Isto implica que $|C(n+1)-C(n)| \leq \beta_{n}|C(n)|$ para $n=1,2, \cdots$, com $\sum_{n=1}^{+\infty} \beta_{n}<\infty$. Assim temos que

$$
\frac{|C(n+1)|}{|C(n)|} \leq 1+\beta_{n}
$$

Como $\sum_{n=1}^{+\infty} \beta_{n}<\infty$ segue que $\Pi_{n \geq n_{0}}\left(1+\beta_{n}\right)<\infty$ e, por comparação, segue que $\Pi_{n \geq n_{0}} \frac{C(n+1)}{C(n)}$ converge, de modo que $C(n) \rightarrow C^{*} \neq 0$ quando $n \rightarrow+\infty$. A estimativa acima para $z(t)$ mostra que $C(t) \rightarrow C^{*}$ quando $t \rightarrow+\infty$ e, portanto, $z(t) \rightarrow C^{*} \psi_{j}^{+}$, o que prova a primeira parte do teorema.

Agora, seja $\hat{w}=w-C^{*} w_{j}^{+}$; esta é uma solução de (1.25) tal que

$$
\|\hat{w}(t)\|_{X}=o\left(\exp \left(\int_{0}^{t} \lambda_{j}(s) d s\right)\right)
$$

quando $t \rightarrow+\infty$. Segue que $\|\hat{w}(t)\|=o\left(e^{t\left(\lambda_{j}^{+}+\varepsilon\right)}\right)$ quando $t \rightarrow+\infty$, para qualquer $\varepsilon>0$, e, por iteração, produzimos a expansão $w(t)=\sum_{j=1}^{m} C_{j} w_{j}^{+}(t)+o\left(e^{-k t}\right)$, para qualquer constante $k$. 
Finalmente, note que provamos apenas convergência em $X$; entretanto, usando o mesmo procedimento adotado na demonstração do Teorema 1.7, podemos mostrar que a convergência também ocorre na norma $\|.\|_{\alpha}$, para qualquer $\alpha<1$, o que implica a convergência em $C^{1}([0,1]) \times C^{1}([0,1])$.

Teorema 1.9 Com as mesmas hipóteses e notações do Teorema 1.7, seja $w=(u, v)$ uma solução de (1.25) tal que, para algum real $k,\|w(t)\|_{X}=O\left(e^{-k t}\right)$ quando $t \rightarrow-\infty$. Se $m$ é escolhido de modo que $\lambda_{m+1}^{-}<-k$, então existem constantes $C_{1}, C_{2}, \cdots, C_{m}$ tais que

$$
w(t)=\sum_{j=1}^{m} C_{j} w_{j}^{-}(t)
$$

$\left(\right.$ Se $\left.k<-\lambda_{1}^{-}, w \equiv 0\right)$.

Demonstração. Aumente $k$ ligeiramente de modo que $\lambda_{m+1}^{-}<-k<\lambda_{m}^{-}$. Seja $\tilde{w}(t)=$ $e^{k t} w(t)$; então $\tilde{w}(t)=(\tilde{u}(t), \tilde{v}(t))$ é limitada para $t \rightarrow-\infty$ e satisfaz

$$
\begin{cases}\tilde{u}_{t}=\left(a(x) \tilde{u}_{x}\right)_{x}+(c(x, t)+k) \tilde{u}, & x \in(0,1) \\ \tilde{v}_{t}=\left(b(x) \tilde{v}_{x}\right)_{x}+(d(x, t)+k) \tilde{v}, & x \in(0,1),\end{cases}
$$

com as mesmas condições de fronteira de (1.25). Assim é suficiente considerarmos o caso $k=0$, com $\lambda_{n+1}^{-}<0<\lambda_{n}^{-}$. Para $t_{0}>0$ suficientemente grande, a teoria básica de variedade estável mostra que o espaço das soluções limitadas em $-\infty<t<-t_{0}$ tem dimensão $n$. Claramente, $w_{1}^{-}, \cdots, w_{n}^{-}$são linearmente independentes, já que do Teorema 1.7

$$
w_{j}^{-}(t)=\exp \left(-\int_{t}^{0} \lambda_{j}(s) d s\right)\left[\psi_{j}^{-}+o(1)\right] \text { quando } t \rightarrow-\infty .
$$

Assim, $\left\{w_{j}^{-}: 1 \leq j \leq n\right\}$ é uma base para este espaço e, consequentemente, existem constantes $c_{1}, \cdots, c_{n}$ tais que

$$
w(t)=\sum_{j=1}^{n} c_{j} w_{j}^{-}(t)
$$

Se $\lambda_{1}^{-}<0$, tem-se $n=0$ e, portanto, $w \equiv 0$. 


\section{Capítulo 2}

\section{Estudo do número de zeros de soluções de equações lineares}

Para uma dada $\phi \in C([-1,1])$ definimos, como em [1], o número de mudança de sinal

$$
\begin{aligned}
\mathcal{S}(\phi)= & \sup \left\{k \geq 0: \text { existem }-1<t_{0}<t_{1}<\cdots<t_{k}<1\right. \\
& \text { tais que } \left.\phi\left(t_{j}\right) \phi\left(t_{j-1}\right)<0, j=1, \cdots, k\right\} .
\end{aligned}
$$

Quando o supremo não existe definimos $\mathcal{S}(\phi)$ como sendo $\infty$.

Enunciamos a seguir, o chamado princípio de Matano para equações parabólicas lineares unidimensionais, que nos dá o comportamento de $\mathcal{S}(u(., t))$ quando $u$ é uma solução de uma equação parabólica linear unidimensional. A prova deste resultado pode ser encontrada em [14] (Lema 2.6) e é uma consequência do princípio do máximo (ver [16] Cap. 3).

Teorema 2.1 Seja $u(x, t)$ uma solução clássica do problema

$$
\left\{\begin{array}{l}
u_{t}=a(x, t) u_{x x}+b(x, t) u_{x}+c(x, t) u, x \in(-1,1), t_{0}<t \leq t_{1} \\
u(-1, t)=u(1, t)=0
\end{array}\right.
$$

onde $a, b$ e c são funções contínuas em $[-1,1] \times\left[t_{0}, t_{1}\right]$. Suponha que existe uma constante positiva $M$ tal que

$$
M^{-1} \leq a(x, t) \leq M, \quad-1 \leq x \leq 1, \quad t_{0} \leq t \leq t_{1},
$$

isto é, a equação é uniformemente parabólica. Então $\mathcal{S}\left(u\left(t_{1}\right)\right) \leq \mathcal{S}\left(u\left(t_{0}\right)\right)$. 
Em outras palavras, o número de mudanças de sinal de uma solução de uma equação parabólica linear não pode crescer com o tempo. Esta é uma propriedade importante das soluções de equações parabólicas lineares, que é de fundamental importância para se demonstrar a transversalidade das variedades estável e instável de pontos de equilíbrio de problemas parabólicos semi-lineares unidimensionais.

O objetivo deste capítulo é demonstrar um resultado equivalente ao Teorema 2.1 para as soluções clássicas do problema

$$
\left\{\begin{array}{l}
u_{t}=\left(p(x) u_{x}\right)_{x}+q(x, t) v, \quad x \in(-1,0) \cup(0,1), t_{0}<t \leq t_{1} \\
u(0-, t)=u(0+, t) \\
p(0-) u_{x}(0-, t)=p(0+) u_{x}(0+, t) \\
u(-1, t)=u(1, t)=0
\end{array}\right.
$$

onde as funções reais $g$ e $q$ estão definidas por

$$
p(x)=\left\{\begin{array}{l}
a(-x), x \in(-1,0) \\
b(x), x \in(0,1)
\end{array} \quad \text { e } q(x, t)=\left\{\begin{array}{l}
c(-x, t), \quad(x, t) \in(-1,0) \times\left[t_{0}, t_{1}\right] \\
d(x, t), \quad(x, t) \in(0,1) \times\left[t_{0}, t_{1}\right]
\end{array}\right.\right.
$$

sendo $a, b \in C^{1}([0,1],(0, \infty))$ e $c(x, t), d(x, t)$ são funções contínuas sobre $[0,1] \times\left[t_{0}, t_{1}\right]$, assim, $g$ é uma função de classe $C^{1}$ em $(-1,0) \cup(0,1)$ estritamente positiva, limitada e $q(x, t)$ é uma função contínua e limitada em $((-1,0) \cup(0,1)) \times\left[t_{0}, t_{1}\right]$.

A irregularidade das soluções do problema $(2.2)$ nos pontos $(x, t)=(0, t)$ nos impede de usar o princípio do máximo na forma apresentada em [16], Cap. 3 . Por esse motivo, não podemos repetir o que foi feito por Matano em [14](Lema 2.6). Foi com sugestões do Prof. Dr. Alexandre Nolasco, que conseguimos contornar as dificuldades utilizando um método geral de aproximações [5]. A base do argumento é aproximarmos, em um certo sentido, as soluções do problema irregular (2.2) por soluções de problemas parabólicos regulares e, para estes, aplicar o Teorema 2.1. Com essa aproximação das soluções conseguimos estabelecer um resultado sobre o número de zeros das soluções lineares, que permite demonstrar, juntamente com os resultados do Capítulo 1, a transversalidade das variedades estável e instável de pontos de equilíbrio do problema (0.1)-(0.3). 


\subsection{Problema aproximado}

Para $\varepsilon \in[0,1]$, consideremos uma família de funções $a_{\varepsilon}:[-1,1] \rightarrow \mathbb{R}$ que satisfaz as seguintes condições :

(i) existe uma constante positiva $M$ tal que $1 / M \leq a_{\varepsilon}(x) \leq M$, para todo $x \in[-1,1]$ e $\varepsilon>0$;

(ii) para $\varepsilon>0, a_{\varepsilon}$ é de classe $C^{1}$ e $a_{\varepsilon}(x) \rightarrow a_{0}(x)$ quando $\varepsilon \rightarrow 0$, para cada $x \in$ $(-1,0) \cup(0,1)$;

(iii) para todo subintervalo fechado $I \subset(-1,0) \cup(0,1), a_{\varepsilon} \rightarrow a_{0}$ e $a_{\varepsilon}^{\prime} \rightarrow a_{0}^{\prime}$ quando $\varepsilon \rightarrow 0$, uniformemente em $I$.

Observe que as hipóteses acima implicam que $a_{0}$ é uma função de classe $C^{1}$ em $(-1,0) \cup$ $(0,1)$, estritamente positiva e limitada em $[-1,1]$.

Agora, consideremos a família de operadores não limitados, positivos e autoadjuntos, e, portanto, setoriais $A_{\varepsilon}: D\left(A_{\varepsilon}\right) \subset L^{2}(-1,1) \rightarrow L^{2}(-1,1)$, definida por

$$
A_{\varepsilon} u(x)=-\left(a_{\varepsilon}(x) u_{x}(x)\right)_{x}, \quad x \in(-1,1), \quad \varepsilon>0,
$$

sendo $D\left(A_{\varepsilon}\right)=\left\{u \in H_{0}^{1}(-1,1):\left(a_{\varepsilon}(x) u_{x}\right)_{x} \in L^{2}(-1,1)\right\}=H^{2}(-1,1) \cap H_{0}^{1}(-1,1)$, e para $\varepsilon=0, A_{0}: D\left(A_{0}\right) \subset L^{2}(-1,1) \rightarrow L^{2}(-1,1)$ é definido por

$$
A_{0} u(x)=-\left(a_{0}(x) u_{x}(x)\right)_{x}, \quad x \in(-1,0) \cup(0,1),
$$

onde

$$
\begin{aligned}
D\left(A_{0}\right) & =\left\{u \in H_{0}^{1}(-1,1):\left(a_{0}(x) u_{x}\right)_{x} \in L^{2}(-1,1), a_{0}(0-) u_{x}(0-)=a_{0}(0+) u_{x}(0+)\right\} \\
& =\left\{u \in H^{2}((-1,0) \cup(0,1)) \cap H_{0}^{1}(-1,1): a_{0}(0-) u_{x}(0-)=a_{0}(0+) u_{x}(0+)\right\} .
\end{aligned}
$$

Denotemos os espaços de potência fracionária de $A_{\varepsilon}$ por $X_{\varepsilon}^{\alpha}, \alpha \geq 0$. Não é difícil verificar que $X_{\varepsilon}^{1 / 2}=H_{0}^{1}(-1,1)$ para todo $\varepsilon \in[0,1]$.

Consideremos ainda uma família de funções $b_{\varepsilon}:[-1,1] \times\left[t_{0}, t_{1}\right] \rightarrow \mathbb{R}, \varepsilon \in[0,1]$, satisfazendo:

(I) existe uma constante positiva $K$, independente de $\varepsilon$, tal que $\left|b_{\varepsilon}(x, t)\right| \leq K$, para todo $(x, t) \in[-1,1] \times\left[t_{0}, t_{1}\right]$ 
(II) para $\varepsilon>0, b_{\varepsilon}$ é contínua, Hölder contínua em $t$, uniformemente para $x \in[-1,1]$, e $b_{\varepsilon}(x, t) \rightarrow b_{0}(x, t)$ quando $\varepsilon \rightarrow 0$, para cada $x \in(-1,0) \cup(0,1)$ e $t \in\left[t_{0}, t_{1}\right]$;

(III) para todo subintervalo fechado $I \subset(-1,0) \cup(0,1), b_{\varepsilon}(\cdot, t) \rightarrow b_{0}(\cdot, t)$ quando $\varepsilon \rightarrow 0$, uniformemente em $I$.

Associadas às funções $b_{\varepsilon}$, consideremos as aplicações $t \in\left[t_{0}, t_{1}\right] \mapsto B_{\varepsilon}(t) \in \mathcal{L}\left(L^{2}(-1,1)\right)$ dadas por

$$
B_{\varepsilon}(t) v(x)=b_{\varepsilon}(x, t) v(x), \text { para toda } v \in L^{2}(-1,1) .
$$

Com as hipóteses adotadas para as funções $b_{\varepsilon}$, temos:

(a) para cada $\varepsilon \geq 0$, as aplicações $t \in\left[t_{0}, t_{1}\right] \longmapsto B_{\varepsilon}(t) \in \mathcal{L}\left(L^{2}\right)$ são Hölder contínuas;

(b) para toda $v \in L^{2}(-1,1)$ e para todo $t \in\left[t_{0}, t_{1}\right], B_{\varepsilon}(t) v \rightarrow B_{0}(t) v$ quando $\varepsilon \rightarrow 0$ em $L^{2}(-1,1)$

(c) existe uma constante $K$ independente de $\varepsilon$ tal que $\sup _{t_{0} \leq t \leq t_{1}}\left\|B_{\varepsilon}(t)\right\|_{\mathcal{L}\left(L^{2}\right)} \leq K$;

Para qualquer $u_{0} \in L^{2}(-1,1)$ podemos considerar a seguinte família de problemas parabólicos lineares não-autônomos

$$
\left\{\begin{array}{l}
u_{t}^{\varepsilon}=-A_{\varepsilon} u^{\varepsilon}+B_{\varepsilon}(t) u^{\varepsilon}, t_{0}<t \leq t_{1} \\
u^{\varepsilon}\left(t_{0}\right)=u_{0}
\end{array}\right.
$$

Seja $\left\{T_{\varepsilon}\left(t, t_{0}\right), t_{0} \leq t \leq t_{1}\right\} \subset \mathcal{L}\left(L^{2}\right)$ a família de operadores de evolução dada pela equação (2.3), no sentido do Teorema 7.1.3 em Henry [11], de modo que $u^{\varepsilon}(t)=$ $T_{\varepsilon}(t, s) u^{\varepsilon}(s)$ quando $u^{\varepsilon}$ resolve a equação (2.3) no intervalo $[s, t]$.

O objetivo agora, é demonstrar a convergência de $u^{\varepsilon}(t)=T_{\varepsilon}\left(t, t_{0}\right) u_{0}$ para $u^{0}(t)=$ $T_{0}\left(t, t_{0}\right) u_{0}$ em $L^{2}(-1,1)$ quando $\varepsilon \rightarrow 0$, para $t_{0}, t$ e $u_{0}$ fixados.

\subsection{Convergência espectral, estimativas para o re- solvente e convergência do resolvente}

Nesta seção analisamos a convergência do operador resolvente e a convergência espectral da família de operadores $A_{\varepsilon}$ quando $\varepsilon \rightarrow 0$.

De [5] temos as seguintes definições : 
Definição 2.1 Dizemos que uma familia de operadores $\left\{B_{\varepsilon} \in \mathcal{L}(X): \varepsilon \in[0,1]\right\}$ é coletivamente compacta se para qualquer família limitada $\left\{u_{\varepsilon} \in X: \varepsilon \in[0,1]\right\}$ existe uma sequência $\varepsilon_{n} \rightarrow 0$ tal que $\left\{B_{\varepsilon_{n}} u_{\varepsilon_{n}}\right\}_{n}$ converge.

Definição 2.2 Dizemos que uma família de operadores $\left\{B_{\varepsilon} \in \mathcal{L}(X): \quad \varepsilon \in[0,1]\right\}$ converge compactamente para $B_{0}$ quando $\varepsilon \rightarrow 0$ se ela é uma familia coletivamente compacta de operadores tais que $B_{\varepsilon}$ é compacto, para todo $\varepsilon \in[0,1]$, e $B_{\varepsilon} \rightarrow B_{0}$ em $\mathcal{L}(X)$, quando $\varepsilon \rightarrow 0$.

Demonstra-se facilmente que os operadores $A_{\varepsilon}$, definidos na seção anterior, têm resolvente compacto, para cada $\varepsilon \in[0,1]$. Os dois lemas a seguir nos permitirão concluir que a família de operadores $\left\{A_{\varepsilon}^{-1} \in \mathcal{L}\left(L^{2}(-1,1)\right): \varepsilon \in[0,1]\right\}$ converge compactamente para $A_{0}^{-1}$.

Lema $2.1 A_{\varepsilon}^{-1} \longrightarrow A_{0}^{-1}$ em $\mathcal{L}\left(L^{2}(-1,1)\right)$, quando $\varepsilon \rightarrow 0$.

Demonstração. Para $\varphi \in L^{2}(-1,1)$ com $\|\varphi\|_{L^{2}}=1$, e $\varepsilon \in[0,1]$, considere $v^{\varepsilon} \in D\left(A_{\varepsilon}\right)$ tal que $A_{\varepsilon} v^{\varepsilon}=\varphi$, ou seja, $v^{\varepsilon}=A_{\varepsilon}^{-1} \varphi$. Precisamos mostrar que

$\left\|v^{\varepsilon}-v^{0}\right\|_{L^{2}(-1,1)}=\left\|A_{\varepsilon}^{-1} \varphi-A_{0}^{-1} \varphi\right\|_{L^{2}(-1,1)} \leq \sup _{\varphi \in L^{2},\|\varphi\|=1}\left\|A_{\varepsilon}^{-1} \varphi-A_{0}^{-1} \varphi\right\|_{L^{2}(-1,1)} \longrightarrow 0$ quando $\varepsilon \rightarrow 0$.

Para $\varepsilon>0$, temos $\left(a_{\varepsilon}(x) v_{x}^{\varepsilon}(x)\right)_{x}=\varphi(x), x \in(-1,1)$. Integrando em $[-1, x]$, duas vezes, obtemos

$$
v^{\varepsilon}(x)-v^{\varepsilon}(-1)=a_{\varepsilon}(-1) v_{x}^{\varepsilon}(-1) \int_{-1}^{x} \frac{1}{a_{\varepsilon}(s)} d s+\int_{-1}^{x} \frac{1}{a_{\varepsilon}(s)}\left(\int_{-1}^{s} \varphi(\tau) d \tau\right) d s .
$$

Lembrando que $v^{\varepsilon} \in H_{0}^{1}(-1,1)$ e integrando por partes o último termo,

$v^{\varepsilon}(x)=a_{\varepsilon}(-1) v_{x}^{\varepsilon}(-1) \int_{-1}^{x} \frac{d s}{a_{\varepsilon}(s)}+\left(\int_{-1}^{x} \frac{d s}{a_{\varepsilon}(s)}\right)\left(\int_{-1}^{x} \varphi(s) d s\right)-\int_{-1}^{x}\left(\int_{-1}^{s} \frac{d \tau}{a_{\varepsilon}(\tau)}\right) \varphi(s) d s$.

Definindo a função contínua

$$
\alpha_{\varepsilon}(x)=\int_{-1}^{x} \frac{d s}{a_{\varepsilon}(s)},-1 \leq x \leq 1,
$$

a última expressão se reescreve

$$
v^{\varepsilon}(x)=a_{\varepsilon}(-1) v_{x}^{\varepsilon}(-1) \alpha_{\varepsilon}(x)+\int_{-1}^{x}\left[\alpha_{\varepsilon}(x)-\alpha_{\varepsilon}(s)\right] \varphi(s) d s, \quad-1 \leq x \leq 1 .
$$


Agora,

$$
0=v^{\varepsilon}(1)=a_{\varepsilon}(-1) v_{x}^{\varepsilon}(-1) \alpha_{\varepsilon}(1)+\int_{-1}^{1}\left[\alpha_{\varepsilon}(1)-\alpha_{\varepsilon}(s)\right] \varphi(s) d s
$$

ou seja,

$$
v_{x}^{\varepsilon}(-1)=-\frac{1}{a_{\varepsilon}(-1) \alpha_{\varepsilon}(1)}+\int_{-1}^{1}\left[\alpha_{\varepsilon}(1)-\alpha_{\varepsilon}(s)\right] \varphi(s) d s
$$

Portanto,

$$
v^{\varepsilon}(x)=\int_{-1}^{1}\left[\frac{\alpha_{\varepsilon}(x) \alpha_{\varepsilon}(s)}{\alpha_{\varepsilon}(1)}-\alpha_{\varepsilon}(x)\right] \varphi(s) d s+\int_{-1}^{x}\left[\alpha_{\varepsilon}(x)-\alpha_{\varepsilon}(s)\right] \varphi(s) d s,
$$

para todo $-1 \leq x \leq 1$.

Procedendo da mesma maneira, porém integrando em $[x, 1]$, obtemos uma outra forma equivalente de explicitarmos $v^{\varepsilon}$, dada por

$$
v^{\varepsilon}(x)=\int_{-1}^{1}\left[\frac{\beta_{\varepsilon}(x) \beta_{\varepsilon}(s)}{\beta_{\varepsilon}(-1)}-\beta_{\varepsilon}(x)\right] \varphi(s) d s+\int_{x}^{1}\left[\beta_{\varepsilon}(x)-\beta_{\varepsilon}(s)\right] \varphi(s) d s,
$$

para todo $-1 \leq x \leq 1$, onde $\beta_{\varepsilon}(x)=\int_{x}^{1} \frac{d s}{a_{\varepsilon}(s)}=\alpha_{\varepsilon}(1)-\alpha_{\varepsilon}(x)$.

Por sua vez, $v^{0} \in D(A)$ satisfaz

$$
\left(a_{0}(x) v_{x}^{0}(x)\right)_{x}=\varphi(x), \quad x \in(-1,0) \cup(0,1)
$$

Como para o caso $\varepsilon>0$, se $x \in(-1,0)$ então integrando (2.6) de -1 a $x$, duas vezes, obtemos

$$
v^{0}(x)=a_{0}(-1) v_{x}^{0}(-1) \alpha_{0}(x)+\int_{-1}^{x}\left[\alpha_{0}(x)-\alpha_{0}(s)\right] \varphi(s) d s
$$

para todo $-1 \leq x<0$. Por outro lado, se $x \in(0,1)$ então

$$
v^{0}(x)=-a_{0}(1) v_{x}^{0}(1) \beta_{0}(x)+\int_{x}^{1}\left[\beta_{0}(x)-\beta_{0}(s)\right] \varphi(s) d s
$$

para todo $0<x \leq 1$.

Das equações (2.7) e (2.8) temos

$$
\begin{gathered}
v^{0}(0-)=a_{0}(-1) v_{x}^{0}(-1) \alpha_{0}(0)+\int_{-1}^{0}\left[\alpha_{0}(0)-\alpha_{0}(s)\right] \varphi(s) d s, \\
v^{0}(0+)=-a_{0}(1) v_{x}^{0}(1) \beta_{0}(0)+\int_{0}^{1}\left[\beta_{0}(0)-\beta_{0}(s)\right] \varphi(s) d s \\
a_{0}(0-) v_{x}^{0}(0-)=a_{0}(-1) v_{x}^{0}(-1)+\int_{-1}^{0} \varphi(s) d s
\end{gathered}
$$




$$
a_{0}(0+) v_{x}^{0}(0+)=a_{0}(1) v_{x}^{0}(1)-\int_{0}^{1} \varphi(s) d s .
$$

Como $v$ é contínua em $x=0$ e $a_{0}(0-) v_{x}^{0}(0-)=a_{0}(0+) v_{x}^{0}(0+)$, temos, das equações (2.9), (2.10), (2.11) e (2.12), o seguinte sistema

$$
\left\{\begin{aligned}
a_{0}(-1) \alpha_{0}(0) v_{x}^{0}(-1)+a_{0}(1) \beta_{0} v_{x}^{0}(1)= & \int_{0}^{1}\left[\beta_{0}(0)-\beta_{0}(s)\right] \varphi(s) d s- \\
& -\int_{-1}^{0}\left[\alpha_{0}(0)-\alpha_{0}(s)\right] \varphi(s) d s \\
a_{0}(-1) v_{x}^{0}(-1)-a_{0}(1) v_{x}^{0}(1)= & -\int_{-1}^{0} \varphi(s) d s-\int_{0}^{1} \varphi(s) d s .
\end{aligned}\right.
$$

Lembrando que $\beta_{0}(x)=\alpha_{0}(1)-\alpha_{0}(x),-1 \leq x \leq 1$, reescrevemos o sistema como

$$
\left\{\begin{aligned}
a_{0}(-1) \alpha_{0}(0) v_{x}^{0}(-1)+a_{0}(1) \beta_{0}(0) v_{x}^{0}(1) & =\int_{-1}^{1}\left[\beta_{0}(0)-\beta_{0}(s)\right] \varphi(s) d s \\
a_{0}(-1) v_{x}^{0}(-1)-a_{0}(1) v_{x}^{0}(1) & =-\int_{-1}^{1} \varphi(s) d s .
\end{aligned}\right.
$$

Multiplicando a segunda equação por $\beta_{0}(0)$ e somando com a primeira obtemos

$$
v_{x}^{0}(-1)=-\frac{1}{a_{0}(-1)\left(\alpha_{0}(0)+\beta_{0}(0)\right)} \int_{-1}^{1} \beta_{0}(s) \varphi(s) d s
$$

Multiplicando a segunda equação do sistema por $-\alpha_{0}(0)$ e somando com a primeira obtemos

$$
v_{x}^{0}(1)=\frac{1}{a_{0}(1)\left(\alpha_{0}(0)+\beta_{0}(0)\right)} \int_{-1}^{1} \alpha_{0}(s) \varphi(s) d s .
$$

Substituindo estas expressões em (2.7) e (2.8), lembrando que $\alpha_{0}(0)+\beta_{0}(0)=\alpha_{0}(1)=$ $\beta_{0}(-1)$, obtemos

$$
v^{0}(x)=-\frac{\alpha_{0}(x)}{\alpha_{0}(1)} \int_{-1}^{1} \beta_{0}(s) \varphi(s) d s+\int_{-1}^{x}\left[\alpha_{0}(x)-\alpha_{0}(s)\right] \varphi(s) d s
$$

para todo $-1 \leq x \leq 0$, e

$$
v^{0}(x)=-\frac{\beta_{0}(x)}{\beta_{0}(-1)} \int_{-1}^{1} \alpha_{0}(s) \varphi(s) d s+\int_{x}^{1}\left[\beta_{0}(x)-\beta_{0}(s)\right] \varphi(s) d s
$$

para todo $0 \leq x \leq 1$. 
Primeiramente, para $x \in[-1,0]$, utilizando (2.4) e (2.15) calculamos

$$
\begin{aligned}
v^{\varepsilon}(x)-v^{0}(x) & =\int_{-1}^{1}\left[\frac{\alpha_{\varepsilon}(x) \alpha_{\varepsilon}(s)}{\alpha_{\varepsilon}(1)}-\alpha_{\varepsilon}(x)+\frac{\alpha_{0}(x)}{\alpha_{0}(1)} \beta_{0}(s)\right] \varphi(s) d s+ \\
& +\int_{-1}^{x}\left[\alpha_{\varepsilon}(x)-\alpha_{\varepsilon}(s)-\alpha_{0}(x)+\alpha_{0}(s)\right] \varphi(s) d s \\
& =\int_{-1}^{1}\left[\frac{\alpha_{\varepsilon}(x) \alpha_{\varepsilon}(s)}{\alpha_{\varepsilon}(1)}-\alpha_{\varepsilon}(x)+\alpha_{0}(x)-\frac{\alpha_{0}(x) \alpha_{0}(s)}{\alpha_{0}(1)}\right] \varphi(s) d s+ \\
& +\int_{-1}^{x}\left[\alpha_{\varepsilon}(x)-\alpha_{\varepsilon}(s)-\alpha_{0}(x)+\alpha_{0}(s)\right] \varphi(s) d s \\
& =\int_{-1}^{1} g_{\varepsilon}(s, x) \varphi(s) d s+\int_{-1}^{x} h_{\varepsilon}(s, x) \varphi(s) d s
\end{aligned}
$$

onde $g_{\varepsilon}(s, x)=\frac{\alpha_{\varepsilon}(x) \alpha_{\varepsilon}(s)}{\alpha_{\varepsilon}(1)}-\alpha_{\varepsilon}(x)+\alpha_{0}(x)-\frac{\alpha_{0}(x) \alpha_{0}(s)}{\alpha_{0}(1)},-1 \leq s \leq 1,-1 \leq x \leq 0 \mathrm{e}$ $h_{\varepsilon}(s, x)=\alpha_{\varepsilon}(x)-\alpha_{\varepsilon}(s)-\alpha_{0}(x)+\alpha_{0}(s),-1 \leq s \leq x \leq 0$, são funções contínuas e uniformemente limitadas para $\varepsilon \in[0,1]$.

De acordo com a definição da função $\alpha_{\varepsilon}$, e lembrando que $a_{\varepsilon}(x) \rightarrow a_{0}(x)$ quando $\varepsilon \rightarrow 0$, para $x \in(-1,0) \cup(0,1)$, temos que $\alpha_{\varepsilon}(x) \rightarrow \alpha_{0}(x)$, para todo $-1 \leq x \leq 1$. Isto implica que, para $-1 \leq s, x \leq 1, g_{\varepsilon}(s, x) \rightarrow 0$ e $h_{\varepsilon}(s, x) \rightarrow 0$ quando $\varepsilon \rightarrow 0$.

Assim, para cada $x \in[-1,0]$, temos, do Teorema da Convergência Dominada,

$$
\begin{aligned}
\left|v^{\varepsilon}(x)-v^{0}(x)\right| & \leq \int_{-1}^{1}\left|g_{\varepsilon}(s, x)\right||\varphi(s)| d s+\int_{-1}^{x}\left|h_{\varepsilon}(s, x)\right||\varphi(s)| d s \\
& \leq\left\{\left(\int_{-1}^{1}\left|g_{\varepsilon}(s, x)\right|^{2} d s\right)^{1 / 2}+\left(\int_{-1}^{x}\left|h_{\varepsilon}(s, x)\right|^{2} d s\right)^{1 / 2}\right\}\left(\int_{-1}^{1}|\varphi(s)|^{2} d s\right)^{1 / 2} \\
& \leq\left(\int_{-1}^{1}\left|g_{\varepsilon}(s, x)\right|^{2} d s\right)^{1 / 2}+\left(\int_{-1}^{x}\left|h_{\varepsilon}(s, x)\right|^{2} d s\right)^{1 / 2} \longrightarrow 0,
\end{aligned}
$$

quando $\varepsilon \rightarrow 0$.

Analogamente, para $x \in[0,1]$, utilizando (2.5) e (2.16) calculamos

$$
\begin{aligned}
v^{\varepsilon}(x)-v(x) & =\int_{-1}^{1}\left[\frac{\beta_{\varepsilon}(x) \beta_{\varepsilon}(s)}{\beta_{\varepsilon}(-1)}-\beta_{\varepsilon}(x)+\frac{\beta_{0}(x) \alpha_{0}(s)}{\beta_{0}(-1)}\right] \varphi(s) d s+ \\
& +\int_{x}^{1}\left[\beta_{\varepsilon}(x)-\beta_{\varepsilon}(s)-\beta_{0}(x)+\beta_{0}(s)\right] \varphi(s) d s \\
& =\int_{-1}^{1}\left[\frac{\beta_{\varepsilon}(x) \beta_{\varepsilon}(s)}{\beta_{\varepsilon}(-1)}-\beta_{\varepsilon}(x)+\beta_{0}(x)-\frac{\beta_{0}(x) \beta_{0}(s)}{\beta_{0}(-1)}\right] \varphi(s) d s+ \\
& +\int_{x}^{1}\left[\beta_{\varepsilon}(x)-\beta_{\varepsilon}(s)-\beta_{0}(x)+\beta_{0}(s)\right] \varphi(s) d s \\
& =\int_{-1}^{1} \tilde{g}_{\varepsilon}(s, x) \varphi(s) d s+\int_{x}^{1} \tilde{h}_{\varepsilon}(s, x) \varphi(s) d s
\end{aligned}
$$


onde $\tilde{g}_{\varepsilon}(s, x)=\frac{\beta_{\varepsilon}(x) \beta_{\varepsilon}(s)}{\beta_{\varepsilon}(-1)}-\beta_{\varepsilon}(x)+\beta_{0}(x)-\frac{\beta_{0}(x) \beta_{0}(s)}{\beta_{0}(-1)}$, para $-1 \leq s \leq 1$ e $0 \leq x \leq 1$, e $\tilde{h}_{\varepsilon}(s, x)=\beta_{\varepsilon}(x)-\beta_{\varepsilon}(s)+\beta_{0}(x)-\beta_{0}(s), 0 \leq s \leq x \leq 1$, são funções contínuas $\mathrm{e}$ uniformemente limitadas para $\varepsilon \in[0,1]$.

Da mesma forma, concluímos que para cada $x \in[0,1]$ temos

$$
\left|v^{\varepsilon}(x)-v^{0}(x)\right| \leq\left(\int_{-1}^{1}\left|\tilde{g}_{\varepsilon}(s, x)\right|^{2} d s\right)^{1 / 2}+\left(\int_{-1}^{x}\left|\tilde{h}_{\varepsilon}(s, x)\right|^{2} d s\right)^{1 / 2} \longrightarrow 0,
$$

quando $\varepsilon \rightarrow 0$.

Da limitação, uniforme em $\varepsilon \in[0,1]$, das funções $g_{\varepsilon}, h_{\varepsilon}, \tilde{g}_{\varepsilon}$ e $\tilde{h}_{\varepsilon}$ e do fato de que $\|\varphi\|_{L^{2}}=1$, segue-se que $A_{\varepsilon}^{-1} \varphi-A_{0}^{-1} \varphi=v^{\varepsilon}-v^{0}$ é também uniformemente limitada para $\varepsilon \in[0,1]$ e $x \in(-1,1)$; portanto, podemos aplicar novamente o Teorema da Convergência Dominada para concluir que

$$
\begin{aligned}
\left\|A_{\varepsilon}^{-1}-A_{0}^{-1}\right\|_{\mathcal{L}\left(L^{2}\right)} & =\sup _{\varphi \in L^{2},\|\varphi\|=1}\left\|A_{\varepsilon}^{-1} \varphi-A_{0}^{-1} \varphi\right\|_{L^{2}(-1,1)} \\
& =\sup _{\varphi \in L^{2},\|\varphi\|=1}\left(\int_{-1}^{1}\left|A_{\varepsilon}^{-1} \varphi(x)-A_{0}^{-1} \varphi(x)\right|^{2} d x\right)^{1 / 2} \longrightarrow 0,
\end{aligned}
$$

quando $\varepsilon \rightarrow 0$, como queríamos demonstrar.

Lema 2.2 A familia $\left\{A_{\varepsilon}^{-1}: \varepsilon \in[0,1]\right\} \subset \mathcal{L}\left(L^{2}(-1,1)\right)$ é limitada e coletivamente compacta.

Demonstração. Seja $\varphi \in L^{2}(-1,1)$ e $v^{\varepsilon}=A_{\varepsilon}^{-1} \varphi \in D\left(A_{\varepsilon}\right), \varepsilon \in[0,1]$. Vimos no lema anterior que para todo $\varepsilon \in[0,1]$,

$$
\left\|A_{\varepsilon}^{-1} \varphi\right\|_{L^{2}}=\left\|v^{\varepsilon}\right\|_{L^{2}} \leq k
$$

sendo $k$ uma constante independente de $\varepsilon$; portanto, segue do Princípio da Limitação Uniforme que a família $\left\{\left\|A_{\varepsilon}^{-1}\right\|: \varepsilon \in[0,1]\right\}$ é limitada.

Agora, considere $\left\{\varphi_{\varepsilon}: \varepsilon \in[0,1]\right\} \subset L^{2}(-1,1)$ uma família limitada, e seja $w^{\varepsilon}=A_{\varepsilon}^{-1} \varphi_{\varepsilon}, \varepsilon \in[0,1]$. Para $\varepsilon>0,-\left(a_{\varepsilon}(x) w_{x}^{\varepsilon}(x)\right)_{x}=\varphi_{\varepsilon}(x)$, para todo $x \in(-1,1)$. Multiplicando por $w^{\varepsilon}(x)$ e integrando de -1 a 1 obtemos

$$
\int_{-1}^{1} a_{\varepsilon}(x)\left(w_{x}^{\varepsilon}(x)\right)^{2} d x=\int_{-1}^{1} \varphi_{\varepsilon}(x) w^{\varepsilon}(x) d x .
$$


Aplicando a desigualdade de Cauchy-Schwarz ao lado direito da expressão acima, e lembrando que $0<1 / M \leq a_{\varepsilon}(x)$, obtemos

$$
\int_{-1}^{1}\left(w_{x}^{\varepsilon}(x)\right)^{2} d x \leq M\left\|\varphi_{\varepsilon}\right\|_{L^{2}}\left\|w^{\varepsilon}\right\|_{L^{2}} \leq M\left\|A_{\varepsilon}^{-1}\right\|\left\|\varphi_{\varepsilon}\right\|_{L^{2}}^{2} \leq K
$$

pois $\left\{\left\|A_{\varepsilon}^{-1}\right\|: \varepsilon \in[0,1]\right\}$ é limitada. Da imersão compacta de $H_{0}^{1}(-1,1)$ em $L^{2}(-1,1)$, segue-se que existe uma sequência $\varepsilon_{n} \rightarrow 0$ tal que $\left\{w^{\varepsilon_{n}}\right\}=\left\{A_{\varepsilon_{n}}^{-1} \varphi_{\varepsilon_{n}}\right\}_{n}$ converge.

A seguir demonstramos um resultado abstrato.

Lema 2.3 Suponha que $\left\{B_{\varepsilon} \in \mathcal{L}(X): \varepsilon \in[0,1]\right\}$ converge compactamente para $B_{0}$ quando $\varepsilon \rightarrow 0$ e que $N\left(I-B_{0}\right)=\{0\}$. Então, existem $\varepsilon_{0}>0$ e $C>0$ tais que

$$
\left\|\left(I-B_{\varepsilon}\right)^{-1}\right\|_{\mathcal{L}(X)} \leq C
$$

para todo $\varepsilon \in\left[0, \varepsilon_{0}\right]$.

Demonstração. Como $B_{0} \in \mathcal{L}(X)$ é compacto, a hipótese $N\left(I-B_{0}\right)=\{0\}$ implica que $R\left(I-B_{0}\right)=X$, ou seja, existe $\left(I-B_{0}\right)^{-1} \in \mathcal{L}(X)$. Como $B_{\varepsilon} \rightarrow B_{0}$ em $\mathcal{L}(X)$, existe $\varepsilon_{0}>0$ tal que $I-B_{\varepsilon}$ é inversível, para todo $\varepsilon \in\left[0, \varepsilon_{0}\right]$.

A estimativa (2.17) é equivalente a

$$
\left\|\left(I-B_{\varepsilon}\right) u_{\varepsilon}\right\|_{X} \geq \frac{1}{C}
$$

para todo $\varepsilon \in\left[0, \varepsilon_{0}\right]$ e para todo $u_{\varepsilon} \in X$ com $\left\|u_{\varepsilon}\right\|_{X}=1$.

Suponha por absurdo que (2.18) não seja verdade, isto é, que existe uma sequência $\left\{u_{\varepsilon_{n}}\right\},\left\|u_{\varepsilon_{n}}\right\|_{X}=1$ e $\varepsilon_{n} \rightarrow 0$, tal que $\left\|\left(I-B_{\varepsilon_{n}}\right) u_{\varepsilon_{n}}\right\|_{X} \rightarrow 0$ quando $n \rightarrow+\infty$.

Por hipótese, $\left\{B_{\varepsilon_{n}} u_{\varepsilon_{n}}\right\}_{n}$ possui uma subsequência convergente, que continuaremos denotando $\left\{B_{\varepsilon_{n}} u_{\varepsilon_{n}}\right\}_{n}$, para $u$. Mas $u_{\varepsilon_{n}}-B_{\varepsilon_{n}} u_{\varepsilon_{n}} \rightarrow 0$, portanto $u_{\varepsilon_{n}} \rightarrow u$ em $X$ e $\|u\|_{X}=$ 1. Isto implica que $\left(I-B_{0}\right) u=0$, o que contradiz a hipótese de que $N\left(I-B_{0}\right)=\{0\}$.

Proposição 2.1 Para qualquer $\lambda \in \rho\left(A_{0}\right)$, existe $\varepsilon_{\lambda}>0$ tal que $\lambda \in \rho\left(A_{\varepsilon}\right)$ para todo $\varepsilon \in\left[0, \varepsilon_{\lambda}\right]$ e existe uma constante $C_{\lambda}>0$ tal que

$$
\left\|\left(\lambda-A_{\varepsilon}\right)^{-1}\right\| \leq C_{\lambda}, \quad \text { para todo } \varepsilon \in\left[0, \varepsilon_{\lambda}\right]
$$

Além disso, $\left(\lambda-A_{\varepsilon}\right)^{-1}$ converge compactamente para $\left(\lambda-A_{0}\right)^{-1}$ quando $\varepsilon \rightarrow 0$. 
Demonstração. Como

$$
\lambda-A_{\varepsilon}=-A_{\varepsilon}\left(I-\lambda A_{\varepsilon}^{-1}\right)
$$

e $A_{\varepsilon}^{-1} \rightarrow A_{0}^{-1}$, temos que $\left(I-\lambda A_{\varepsilon}^{-1}\right)$ é inversível, isto é, $\lambda \in \rho\left(A_{\varepsilon}\right)$, para $\varepsilon>0$ suficientemente pequeno.

Agora, fazendo $B_{\varepsilon}=\lambda A_{\varepsilon}^{-1}$ no Lema 2.3 , existem $\varepsilon_{\lambda}>0$ e $\bar{C}_{\lambda}>0$ tais que

$$
\left\|\left(I-\lambda A_{\varepsilon}^{-1}\right)^{-1}\right\| \leq \bar{C}_{\lambda}, \quad \forall \varepsilon \in\left[0, \varepsilon_{\lambda}\right]
$$

o que implica

$$
\left\|\left(\lambda-A_{\varepsilon}\right)^{-1}\right\| \leq\left\|\left(I-\lambda A_{\varepsilon}^{-1}\right)^{-1}\right\|\left\|A_{\varepsilon}^{-1}\right\| \leq C_{\lambda}
$$

para todo $\varepsilon \in\left[0, \varepsilon_{\lambda}\right]$.

Da limitação de $\left\{\left\|\left(I-\lambda A_{\varepsilon}^{-1}\right)^{-1}\right\|: \varepsilon \in\left[0, \varepsilon_{\lambda}\right]\right\}$ e da convergência compacta de $A_{\varepsilon}^{-1}$ para $A_{0}^{-1}$, segue que $\left(\lambda-A_{\varepsilon}\right)^{-1}$ converge compactamente para $\left(\lambda-A_{0}\right)^{-1}$ quando $\varepsilon \rightarrow 0$.

Teorema 2.2 Para cada $\varepsilon \in(0,1]$, sejam

$$
\lambda_{1}^{\varepsilon}<\lambda_{2}^{\varepsilon}<\cdots<\lambda_{k}^{\varepsilon}<\cdots \rightarrow+\infty
$$

os autovalores de $A_{\varepsilon} e\left\{\phi_{k}^{\varepsilon}\right\}_{k}$ as autofunções associadas. Considere

$$
\lambda_{1}^{0}<\lambda_{2}^{0}<\cdots<\lambda_{k}^{0}<\cdots \rightarrow+\infty
$$

os autovalores de $A_{0} e\left\{\phi_{k}^{0}\right\}_{k}$ as autofunções associadas. Então, são verdadeiras as seguintes afirmações :

(i) se para alguma sequência $\varepsilon_{n} \rightarrow 0$ e $\left\{\mu_{n}\right\}_{n}, \mu_{n} \in \sigma\left(A_{\varepsilon_{n}}\right), n \in \mathbb{N}$, tivermos $\mu_{n} \rightarrow \mu_{0}$ quando $n \rightarrow+\infty$, então $\mu_{0} \in \sigma\left(A_{0}\right)$;

(ii) para cada $k \in \mathbb{N}, \lambda_{k}^{\varepsilon} \rightarrow \lambda_{k}^{0}$ quando $\varepsilon \rightarrow 0$;

(iii) para cada $k \in \mathbb{N}$ e para qualquer sequência $\varepsilon_{n} \rightarrow 0$, existe uma subsequência $\varepsilon_{n_{j}}$ tal que

$$
\phi_{k}^{\varepsilon_{n_{j}}} \rightarrow \phi_{k}^{0} \text { em } H_{0}^{1}(-1,1), \text { quando } j \rightarrow+\infty
$$


Demonstração. (i) Como $\mu_{n} \in \sigma\left(A_{\varepsilon_{n}}\right)$, existe uma sequência $\left\{u_{n}\right\}_{n} \subset D\left(A_{\varepsilon_{n}}\right)$, com $\left\|u_{n}\right\|_{L^{2}}=1$, tal que $\left(I-\mu_{n} A_{\varepsilon_{n}}^{-1}\right) u_{n}=0$. Daí,

$$
\left\|\left(I-\mu_{0} A_{\varepsilon_{n}}^{-1}\right) u_{n}\right\|=\left\|\left(I-\mu_{n} A_{\varepsilon_{n}}^{-1}\right) u_{n}-\left(\mu_{0}-\mu_{n}\right) A_{\varepsilon_{n}}^{-1} u_{n}\right\|=\left|\mu_{0}-\mu_{n}\right|\left\|A_{\varepsilon_{n}}^{-1} u_{n}\right\| \rightarrow 0
$$

quando $n \rightarrow \infty$, pois $\left\{\left\|A_{\varepsilon}^{-1}\right\|: \varepsilon \in[0,1]\right\}$ é limitada. Ainda, como $\left\{u_{n}\right\}_{n}$ é limitada, tomando subsequência se necessário, temos que $\mu_{0} A_{\varepsilon_{n}}^{-1} u_{n} \rightarrow u$ em $L^{2}(-1,1)$, o que implica, de (2.19), que $u_{n} \rightarrow u$ em $L^{2}(-1,1),\|u\|_{L^{2}}=1$. Mas $\left(I-\mu_{0} A_{\varepsilon_{n}}^{-1}\right) \rightarrow\left(I-\mu_{0} A_{0}^{-1}\right)$ em $\mathcal{L}\left(L^{2}(-1,1)\right)$, o que implica que

$$
\left(I-\mu_{0} A_{\varepsilon_{n}}^{-1}\right) u_{n} \longrightarrow\left(I-\mu_{0} A_{0}^{-1}\right) u=0
$$

quando $n \rightarrow+\infty$. Portanto $\mu_{0} \in \sigma\left(A_{0}\right)$.

(ii) Para cada $\varepsilon \in[0,1]$, consideremos a forma bilinear simétrica

$$
\tau_{\varepsilon}: H_{0}^{1}(-1,1) \times H_{0}^{1}(-1,1) \longrightarrow \mathbb{R}
$$

associada ao operador $A_{\varepsilon}$, dada por

$$
\tau_{\varepsilon}(u, v)=\int_{-1}^{1} a_{\varepsilon}(x) u^{\prime}(x) v^{\prime}(x) d x
$$

Como $a_{\varepsilon}(x) \rightarrow a_{0}(x)$, para todo $x \in(-1,0) \cup(0,1)$, segue do Teorema da Convergência Dominada que, para cada $(u, v) \in H_{0}^{1}(-1,1) \times H_{0}^{1}(-1,1)$,

$$
\tau_{\varepsilon}(u, v) \rightarrow \tau_{0}(u, v) \text { quando } \varepsilon \rightarrow 0
$$

O primeiro autovalor de $A_{\varepsilon}$ é dado por

$$
\lambda_{1}^{\varepsilon}=\inf _{\substack{u \in H_{0}^{1}(-1,1) \\\|u\|_{L^{2}}=1}} \tau_{\varepsilon}(u, u)
$$

então,

$$
\lambda_{1}^{\varepsilon}=\inf _{\substack{u \in H_{0}^{1}(-1,1) \\\|u\|_{L^{2}}=1}} \tau_{\varepsilon}(u, u) \leq \tau_{\varepsilon}(u, u) \rightarrow \tau_{0}(u, u) \text { quando } \varepsilon \rightarrow 0,
$$

o que implica que

$$
\limsup _{\varepsilon \rightarrow 0} \lambda_{1}^{\varepsilon} \leq \lim _{\varepsilon \rightarrow 0} \tau_{\varepsilon}(u, u)=\tau_{0}(u, u)
$$


para toda $u \in H_{0}^{1}(-1,1)$ com $\|u\|_{L^{2}}=1$. Logo,

$$
\limsup _{\varepsilon \rightarrow 0} \lambda_{1}^{\varepsilon} \leq \inf _{\substack{u \in H_{0}^{1}(-1,1) \\\|u\|_{L^{2}}=1}} \tau_{0}(u, u)=\lambda_{1}^{0} .
$$

Denotemos $\gamma_{1}=\liminf _{\varepsilon \rightarrow 0} \lambda_{1}^{\varepsilon}$. Vamos provar que $\gamma_{1}=\lambda_{1}^{0}$. De fato, da definição de $\gamma_{1}$ existe uma sequência $\varepsilon_{n} \rightarrow 0$ tal que

$$
\lambda_{1}^{\varepsilon_{n}} \longrightarrow \gamma_{1} \leq \limsup _{\varepsilon \rightarrow 0} \lambda_{1}^{\varepsilon} \leq \lambda_{1}^{0} .
$$

Como $\lambda_{1}^{\varepsilon_{n}} \in \sigma\left(A_{\varepsilon_{n}}\right)$ segue, do item (i), que $\gamma_{1} \in \sigma\left(A_{0}\right)$, o que implica, $\gamma_{1}=\lambda_{1}^{0}$, visto que $\gamma_{1} \leq \lambda_{1}^{0}$. Assim,

$$
\lambda_{1}^{0}=\liminf _{\varepsilon \rightarrow 0} \lambda_{1}^{\varepsilon} \leq \limsup _{\varepsilon \rightarrow 0} \lambda_{1}^{\varepsilon} \leq \lambda_{1}^{0}
$$

ou seja,

$$
\lim _{\varepsilon \rightarrow 0} \lambda_{1}^{\varepsilon}=\lambda_{1}^{0} .
$$

Analisemos agora, a convergência das autofunções $\left\{\phi_{1}^{\varepsilon}\right\}_{\varepsilon}$. Temos que,

$$
1 / M\left\|\phi_{1}^{\varepsilon}\right\|_{H_{0}^{1}} \leq \int_{-1}^{1} a_{\varepsilon}(x)\left(\phi_{1 x}^{\varepsilon}(x)\right)^{2} d x=\lambda_{1}^{\varepsilon} \leq K,
$$

para todo $\varepsilon \in[0,1]$, sendo $K$ e $M$ constantes independentes de $\varepsilon$. Da imersão compacta de $H_{0}^{1}(-1,1)$ em $L^{2}(-1,1)$, existe uma sequência $\varepsilon_{n} \rightarrow 0$ tal que $\phi_{1}^{\varepsilon_{n}} \rightarrow \phi_{1}$ na norma de $L^{2}(-1,1)$ e fracamente em $H_{0}^{1}(-1,1)$, quando $n \rightarrow+\infty$, com $\left\|\phi_{1}\right\|_{L^{2}}=1$. Como $\left(I-\lambda_{1}^{\varepsilon_{n}} A_{\varepsilon_{n}}^{-1}\right) \rightarrow\left(I-\lambda_{1}^{0} A_{0}^{-1}\right)$ em $\mathcal{L}\left(L^{2}\right)$, temos

$$
0=\left(I-\lambda_{1}^{\varepsilon_{n}} A_{\varepsilon_{n}}^{-1}\right) \phi_{1}^{\varepsilon_{n}} \longrightarrow\left(I-\lambda_{1}^{0} A_{0}^{-1}\right) \phi_{1}, \text { quando } n \rightarrow+\infty,
$$

o que implica, $\left(I-\lambda_{1}^{0} A_{0}^{-1}\right) \phi_{1}=0$, donde concluímos que $\phi_{1} \in D\left(A_{0}\right)$ é uma autofunção normalizada de $A_{0}$ associada ao autovalor $\lambda_{1}^{0} \in \sigma\left(A_{0}\right)$. Agora, como

$$
\begin{aligned}
\lambda_{1}^{\varepsilon_{n}} & =\int_{0}^{1} a_{\varepsilon_{n}}(x)\left(\phi_{1 x}^{\varepsilon_{n}}(x)\right)^{2} d x \\
& =\int_{0}^{1} a_{\varepsilon_{n}}(x)\left[\left(\phi_{1 x}^{\varepsilon_{n}}(x)\right)^{2}-\left(\phi_{1 x}(x)\right)^{2}\right] d x+\int_{0}^{1} a_{\varepsilon_{n}}(x)\left(\phi_{1 x}(x)\right)^{2} d x \\
& \longrightarrow \int_{0}^{1} a_{0}(x)\left(\phi_{1 x}(x)\right)^{2} d x=\lambda_{1}^{0}
\end{aligned}
$$

e $\int_{0}^{1} a_{\varepsilon_{n}}(x)\left(\phi_{1 x}(x)\right)^{2} d x \rightarrow \int_{0}^{1} a_{0}(x)\left(\phi_{1 x}(x)\right)^{2} d x$, quando $n \rightarrow+\infty$, temos

$$
\int_{0}^{1} a_{\varepsilon_{n}}(x)\left[\left(\phi_{1 x}^{\varepsilon_{n}}(x)\right)^{2}-\left(\phi_{1 x}(x)\right)^{2}\right] d x \rightarrow 0
$$


quando $n \rightarrow+\infty$ o que implica $\left\|\phi_{1}^{\varepsilon_{n}}\right\|_{H_{0}^{1}} \rightarrow\left\|\phi_{1}\right\|_{H_{0}^{1}}$ quando $n \rightarrow+\infty$. Disto e da convergência fraca em $H_{0}^{1}(-1,1)$ segue que $\phi_{1}^{\varepsilon_{n}} \rightarrow \phi_{1}$ na norma de $H_{0}^{1}(-1,1)$. Portanto, o teorema está provado para o caso $k=1$.

Assuma, por indução, que para os $k-1$ primeiros autovalores, tenhamos $\lambda_{j}^{\varepsilon} \rightarrow \lambda_{j}^{0}$ quando $\varepsilon \rightarrow 0$, e que qualquer sequência $\varepsilon_{n} \rightarrow 0$, existe uma subsequência $\left\{\varepsilon_{n_{j}}\right\}_{j}$ tal que

$$
\phi_{i}^{\varepsilon_{n_{j}}} \rightarrow \phi_{i}^{0} \text { em } H_{0}^{1}(-1,1) \text { quando } j \rightarrow+\infty
$$

para todo $i=1, \cdots, k-1$. As autofunções $\phi_{i}^{0}$ são duas a duas ortogonais em $L^{2}(-1,1)$.

Da caracterização min-max dos autovalores temos que

$$
\lambda_{k}^{\varepsilon}=\inf _{\substack{u \in H_{0}^{1}(-1,1) \\ u \perp\left\{\phi_{1}^{\varepsilon}, \cdots, \phi_{k-1}^{\varepsilon}\right\} \\\|u\|_{L^{2}=1}}} \tau_{\varepsilon}(u, u) .
$$

Definimos as seguintes projeções de $L^{2}(-1,1)$ sobre $H_{0}^{1}(-1,1)$

$$
\begin{gathered}
P_{k}^{\varepsilon_{n_{j}}}=\sum_{i=1}^{k-1}\left\langle\phi_{i}^{\varepsilon_{n_{j}}}, .\right\rangle \phi_{i}^{\varepsilon_{n_{j}}}, \quad Q_{k}^{\varepsilon_{n_{j}}}=I-P_{k}^{\varepsilon_{n_{j}}} \\
P_{k}^{0}=\sum_{i=1}^{k-1}\left\langle\phi_{i}^{0}, .\right\rangle \phi_{i}^{0}, \quad Q_{k}^{0}=I-P_{k}^{0} .
\end{gathered}
$$

Da convergência $\phi_{i}^{\varepsilon_{n_{j}}} \rightarrow \phi_{i}^{0}$ em $H_{0}^{1}(-1,1), i=1,2, \cdots, k-1$, segue que

$$
P_{k}^{\varepsilon_{n_{j}}} \longrightarrow P_{k}^{0} \quad \text { e } \quad Q_{k}^{\varepsilon_{n_{j}}} \longrightarrow Q_{k}^{0} \text { em } \mathcal{L}\left(L^{2}, H_{0}^{1}\right)
$$

Para um dado $u \in H_{0}^{1}(-1,1)$, tomemos $u^{\varepsilon_{n_{j}}}=Q_{k}^{\varepsilon_{n_{j}}} u$. Como $u^{\varepsilon_{n_{j}}}$ é ortogonal em $L^{2}(-1,1)$ a $\phi_{1}^{\varepsilon_{n_{j}}}, \cdots, \phi_{k-1}^{\varepsilon_{n_{j}}}$ e converge para $u^{0}=Q_{k}^{0} u$ em $H_{0}^{1}(-1,1)$, mostraremos abaixo que

$$
\lambda_{k}^{\varepsilon_{n_{j}}} \leq \frac{\tau_{\varepsilon_{n_{j}}}\left(u^{\varepsilon_{n_{j}}}, u^{\varepsilon_{n_{j}}}\right)}{\left\|u^{\varepsilon_{n_{j}}}\right\|^{2}} \longrightarrow \frac{\tau_{0}\left(u^{0}, u^{0}\right)}{\left\|u^{0}\right\|^{2}} \text {, quando } j \rightarrow+\infty .
$$

Com isto, como $u^{0} \perp\left\{\phi_{1}^{0}, \cdots, \phi_{k-1}^{0}\right\}$ é arbitrário, obtemos que

$$
\underset{j}{\limsup } \lambda_{k}^{\varepsilon_{n_{j}}} \leq \limsup _{j} \frac{\tau_{\varepsilon_{n_{j}}}\left(u^{\varepsilon_{n_{j}}}, u^{\varepsilon_{n_{j}}}\right)}{\left\|u^{\varepsilon_{n_{j}}}\right\|^{2}}=\frac{\tau_{0}\left(u^{0}, u^{0}\right)}{\left\|u^{0}\right\|^{2}},
$$

o que implica

$$
\limsup _{j} \lambda_{k}^{\varepsilon_{n_{j}}} \leq \lambda_{k}^{0}
$$


Como este limitante superior é independente da sequência $\varepsilon_{n} \rightarrow 0$ e da subsequência $\left\{\varepsilon_{n_{j}}\right\}_{j}$, obtemos

$$
\limsup _{\varepsilon} \lambda_{k}^{\varepsilon} \leq \lambda_{k}^{0}
$$

Vamos provar a afirmativa acima. A primeira desigualdade de (2.22) é imediata. Como $u^{\varepsilon_{n_{j}}}=Q_{k}^{\varepsilon_{n_{j}}} u=u-P_{k}^{\varepsilon_{n_{j}}} u$, temos que $\tau_{\varepsilon_{n_{j}}}\left(u^{\varepsilon_{n_{j}}}, u^{\varepsilon_{n_{j}}}\right)=\tau_{\varepsilon_{n_{j}}}(u, u)-2 \tau_{\varepsilon_{n_{j}}}\left(u, P_{k}^{\varepsilon_{n_{j}}} u\right)+$ $\tau_{\varepsilon_{n_{j}}}\left(P_{k}^{\varepsilon_{n_{j}}} u, P_{k}^{\varepsilon_{n_{j}}} u\right)$. Tomamos $u \in H_{0}^{1}(-1,1)$, então

$$
\tau_{\varepsilon_{n_{j}}}(u, u) \rightarrow \tau_{0}(u, u) \text { quando } j \rightarrow+\infty
$$

Também,

$$
\begin{aligned}
\tau_{\varepsilon_{n_{j}}}\left(u, P_{k}^{\varepsilon_{n_{j}}} u\right)=\int_{-1}^{1} a_{\varepsilon_{n_{j}}}(x) u_{x}(x)\left(P_{k}^{\varepsilon_{n_{j}}} u\right)_{x}(x) d x & \rightarrow \int_{-1}^{1} a_{0}(x) u_{x}(x) u_{x}(x)\left(P_{k}^{0} u\right)_{x}(x) d x \\
& =\tau_{0}\left(u, P_{k}^{0} u\right)
\end{aligned}
$$

Finalmente,

$$
\tau_{\varepsilon_{n_{j}}}\left(P_{k}^{\varepsilon_{n_{j}}} u, P_{k}^{\varepsilon_{n_{j}}} u\right)=\sum_{i=1}^{k-1}\left|\left\langle\phi_{i}^{\varepsilon_{n_{j}}}, u\right\rangle\right|^{2} \tau_{\varepsilon_{n_{j}}}\left(\phi_{i}^{\varepsilon_{n_{j}}}, \phi_{i}^{\varepsilon_{n_{j}}}\right)
$$

e $\tau_{\varepsilon_{n_{j}}}\left(\phi_{i}^{\varepsilon_{n_{j}}}, \phi_{i}^{\varepsilon_{n_{j}}}\right) \rightarrow \tau_{0}\left(\phi_{i}^{0}, \phi_{i}^{0}\right)$, pois $\phi_{i}^{\varepsilon_{n_{j}}} \rightarrow \phi_{i}^{0}$ em $H_{0}^{1}(-1,1)$ e $\tau_{\varepsilon_{n_{j}}}\left(\phi_{i}^{0}, \phi_{i}^{0}\right) \rightarrow \tau_{0}\left(\phi_{i}^{0}, \phi_{i}^{0}\right)$ quando $j \rightarrow+\infty$. Assim,

$$
\tau_{\varepsilon_{n_{j}}}\left(P_{k}^{\varepsilon_{n_{j}}} u, P_{k}^{\varepsilon_{n_{j}}} u\right) \longrightarrow \sum_{i=1}^{k-1}\left|\left\langle\phi_{i}^{0}, u\right\rangle\right|^{2} \tau_{0}\left(\phi_{i}^{0}, \phi_{i}^{0}\right)=\tau_{0}\left(P_{k}^{0} u, P_{k}^{0}\right) .
$$

Como $u^{0}=Q_{k}^{0} u=u-P_{k}^{0} u, \tau_{0}\left(u^{0}, u^{0}\right)=\tau_{0}(u, u)-2 \tau_{0}\left(u, P_{k}^{0} u\right)+\tau_{0}\left(P_{k}^{0} u, P_{k}^{0} u\right)$ e por (2.24), (2.25) e (2.26), segue que

$$
\tau_{\varepsilon_{n_{j}}}\left(u^{\varepsilon_{n_{j}}}, u^{\varepsilon_{n_{j}}}\right) \longrightarrow \tau_{0}\left(u^{0}, u^{0}\right) \text { quando } j \rightarrow+\infty
$$

Como consequência de $(2.22)$, tomando a família da $k$-ésima autofunção $\left\{\phi_{k}^{\varepsilon_{n_{j}}}\right\}_{j}$, temos

$$
\tau_{\varepsilon_{n_{j}}}\left(\phi_{k}^{\varepsilon_{n_{j}}}, \phi_{k}^{\varepsilon_{n_{j}}}\right)=\lambda_{k}^{\varepsilon_{n_{j}}} \leq K
$$

para alguma constante $K$ independente de $j \in \mathbb{N}$. Portanto, $\int_{-1}^{1} a_{\varepsilon_{n_{j}}}(x)\left[\phi_{k x}^{\varepsilon_{n_{j}}}(x)\right]^{2} d x$ é limitada. Da imersão compacta de $H_{0}^{1}(-1,1)$ em $L^{2}(-1,1)$, existe uma subsequência de $\left\{\varepsilon_{n_{j}}\right\}_{j}$, a qual continuaremos denotando da mesma forma, tal que

$$
\lambda_{k}^{\varepsilon_{n_{j}}} \rightarrow \mu_{k} \quad \text { e } \quad \phi_{k}^{\varepsilon_{n_{j}}} \rightarrow \phi_{k} \quad \text { em } \quad L^{2}(-1,1)
$$


quando $j \rightarrow+\infty$. Como $\left(I-\lambda_{k}^{\varepsilon_{n_{j}}} A_{\varepsilon_{n_{j}}}^{-1}\right) \rightarrow\left(I-\mu_{k} A_{0}^{-1}\right)$ em $\mathcal{L}\left(L^{2}\right)$ e $\left(I-\lambda_{k}^{\varepsilon_{n_{j}}} A_{\varepsilon_{n_{j}}}^{-1}\right) \phi_{k}^{\varepsilon_{n_{j}}}=0$ segue que $\left(I-\mu_{k} A_{0}^{-1}\right) \phi_{k}=0$, ou seja, $A_{0} \phi_{k}=\mu_{k} \phi_{k}$ e $\tau_{0}\left(\phi_{k}, \phi_{k}\right)=\mu_{k}$.

Ainda, como $\phi_{k}^{\varepsilon_{n_{j}}}$ e $\phi_{i}^{\varepsilon_{n_{j}}}$ são ortogonais em $L^{2}(-1,1)$ para todo $i=1, \cdots, k-1$, temos que $\phi_{k}$ e $\phi_{i}^{0}$ também são ortogonais em $L^{2}(-1,1)$, para todo $i=1, \cdots, k-1$. Consequentemente,

$$
\lambda_{k}^{0}=\inf _{\substack{u \in H_{0}^{1}(-1,1) \\ u \perp\left\{\phi_{1}^{0}, \cdots, \phi_{k-1}^{0}\right\} \\\|u\|_{L^{2}=1}}} \tau_{0}(u, u) \leq \tau_{0}\left(\phi_{k}, \phi_{k}\right)=\mu_{k}=\lim _{j} \lambda_{k}^{\varepsilon_{n_{j}}}
$$

Como o limitante inferior é independente da sequência $\varepsilon_{n} \rightarrow 0$ e da subsequência $\left\{\varepsilon_{n_{j}}\right\}_{j}$, obtemos

$$
\lambda_{k}^{0} \leq \liminf _{\varepsilon} \lambda_{k}^{\varepsilon}
$$

o que nos permite concluir, juntamente com (2.23), que

$$
\lim _{\varepsilon} \lambda_{k}^{\varepsilon}=\lambda_{k}^{0}
$$

Da mesma forma que provamos a convergência na norma de $H_{0}^{1}(-1,1)$ da sequência $\left\{\phi_{1}^{\varepsilon_{n}}\right\}_{n}$, podemos provar que $\phi_{k}^{\varepsilon_{n_{j}}} \rightarrow \phi_{k}$ em $H_{0}^{1}(-1,1)$ quando $j \rightarrow+\infty$, lembrando que $\phi_{k}$ é uma autofunção normalizada de $A_{0}$ associada ao autovalor $\lambda_{k}^{0}$.

Lema 2.4 Seja $K \subset \rho\left(A_{0}\right)$ um subconjunto compacto. Então, existe $\varepsilon_{K}>0$ tal que $K \subset \rho\left(A_{\varepsilon}\right)$ para todo $\varepsilon \in\left[0, \varepsilon_{K}\right] e$

$$
\sup _{\substack{\lambda \in K \\ \varepsilon \in\left[0, \varepsilon_{K}\right]}}\left\|\left(\lambda-A_{\varepsilon}\right)^{-1}\right\|<\infty .
$$

Além disso,

$$
\sup _{\lambda \in K}\left\|\left(\lambda-A_{\varepsilon}\right)^{-1}-(\lambda-A)^{-1}\right\| \rightarrow 0 \text { quando } \varepsilon \rightarrow 0 .
$$

Demonstração. Primeiramente, vamos provar que existe um $\varepsilon_{K}>0$ tal que $K \subset \rho\left(A_{\varepsilon}\right)$ para todo $\varepsilon \in\left[0, \varepsilon_{K}\right]$. Suponha, por absurdo, que isto não seja verdade; então existem sequências $\varepsilon_{n} \rightarrow 0,\left\{\lambda_{n}\right\}_{n} \subset K$ tal que $\lambda_{n}$ é um autovalor de $A_{\varepsilon_{n}}$. Como $K$ é compacto, 
podemos supor que $\lambda_{n} \rightarrow \bar{\lambda} \in K$. Segue do Teorema 2.2, parte (i), que $\bar{\lambda} \in \sigma\left(A_{0}\right)$, o que é uma contradição. Para provar (2.27), basta provar que

$$
\sup _{\substack{\lambda \in K \\ \varepsilon \in\left[0, \varepsilon_{K}\right]}}\left\|\left(I-\lambda A_{\varepsilon}^{-1}\right)^{-1}\right\|<\infty .
$$

Suponhamos que este não seja o caso, isto é, que existam sequências $\varepsilon_{n} \rightarrow 0,\left\{\lambda_{n}\right\}_{n} \subset K$ (que podemos assumir convergente para $\bar{\lambda} \in K$ ) tal que

$$
\left\|\left(I-\lambda_{n} A_{\varepsilon_{n}}^{-1}\right)^{-1}\right\| \rightarrow \infty, \text { quando } n \rightarrow+\infty .
$$

Como $\lambda_{n} A_{\varepsilon_{n}}^{-1}$ converge compactamente para $\bar{\lambda} A_{0}^{-1}$, segue do Lema 2.3 que isto é uma contradição.

Provemos agora (2.28). Suponha por absurdo, que existem sequências $\varepsilon_{n} \rightarrow 0$, $\left\{\lambda_{n}\right\}_{n} \subset K, \lambda_{n} \rightarrow \bar{\lambda} \in K$, e $\delta>0$ tais que

$$
\left\|\left(\lambda_{n}-A_{\varepsilon_{n}}\right)^{-1}-\left(\lambda_{n}-A_{0}\right)^{-1}\right\| \geq \delta .
$$

Usando a identidade do resolvente

$$
\left(\lambda_{n}-A_{\varepsilon_{n}}\right)^{-1}-\left(\bar{\lambda}-A_{\varepsilon_{n}}\right)^{-1}=\left(\bar{\lambda}-\lambda_{n}\right)\left(\lambda_{n}-A_{\varepsilon_{n}}\right)^{-1}\left(\bar{\lambda}-A_{\varepsilon_{n}}\right)^{-1},
$$

e (2.27), obtemos

$$
\left\|\left(\lambda_{n}-A_{\varepsilon_{n}}\right)^{-1}-\left(\bar{\lambda}-A_{\varepsilon_{n}}\right)^{-1}\right\| \rightarrow 0 \text { quando } n \rightarrow+\infty .
$$

Da Proposição 2.1, temos

$$
\left\|\left(\bar{\lambda}-A_{\varepsilon_{n}}\right)^{-1}-\left(\bar{\lambda}-A_{0}\right)^{-1}\right\| \rightarrow 0 \text { quando } n \rightarrow+\infty
$$

e da propriedade de continuidade do operador resolvente,

$$
\left\|\left(\lambda_{n}-A\right)^{-1}-\left(\bar{\lambda}-A_{0}\right)^{-1}\right\| \rightarrow 0 \text { quando } n \rightarrow \infty .
$$

Com isso, temos que (2.30), (2.31) e (2.32) estão em contradição com (2.29) e o resultado está provado. 
Lema 2.5 Existem constantes $\delta>0,0<\theta<\pi / 2$ e $C \geq 1$, independentes de $\varepsilon \geq 0$, tais que o setor

$$
\Sigma_{\delta, \theta}=\{\lambda \in \mathbb{C}: \theta<|\arg (\lambda-\delta)| \leq \pi\} \quad \subset \quad \rho\left(\mathrm{A}_{\varepsilon}\right)
$$

$e$

$$
\left\|\left(\lambda-A_{\varepsilon}\right)^{-1}\right\| \leq \frac{C}{1+|\lambda|}, \forall \lambda \in \Sigma_{\delta, \theta}
$$

para todo $\varepsilon \in[0,1]$.

Demonstração. A imagem numérica do operador $A_{\varepsilon}$ é dada por

$$
W\left(A_{\varepsilon}\right)=\left\{\left\langle u, A_{\varepsilon} u\right\rangle: u \in D\left(A_{\varepsilon}\right),\|u\|_{L^{2}}=1\right\} .
$$

Como

$$
\left\langle u, A_{\varepsilon} u\right\rangle=-\int_{-1}^{1}\left(a_{\varepsilon}(x) u_{x}(x)\right)_{x} u_{x}(x) d x=\int_{-1}^{1} a_{\varepsilon}(x)\left(u_{x}(x)\right)^{2} d x \geq \frac{1}{4 M}\|u\|_{L^{2}}^{2},
$$

denotando $\kappa=\frac{1}{4 M}$, segue que $W\left(A_{\varepsilon}\right) \subset[\kappa,+\infty)$. Além disso, como $0 \in \rho\left(A_{\varepsilon}\right)$, segue do Teorema 3.9, Cap. 1 [15] que $\sigma\left(A_{\varepsilon}\right) \subset[\kappa,+\infty)$ e, fixados $0<\delta<\kappa$ e $0<\theta<\pi / 2$, o setor

$$
\Sigma_{\delta, \theta}=\{\lambda \in \mathbb{C}: \theta<|\arg (\lambda-\delta)| \leq \pi\} \quad \subset \rho\left(\mathrm{A}_{\varepsilon}\right)
$$

e

$$
\left\|\left(\lambda-A_{\varepsilon}\right)^{-1}\right\| \leq \frac{1}{d\left(\lambda, \overline{W\left(A_{\varepsilon}\right)}\right)}, \text { para todo } \lambda \in \Sigma_{\delta, \theta} .
$$

Mostraremos que existe uma constante $C$ tal que $d\left(\lambda, \overline{W\left(A_{\varepsilon}\right)}\right) \geq \frac{|\lambda|+1}{C}$, para todo $\lambda \in \Sigma_{\delta, \theta}$.

De fato, para $\lambda \in \Sigma_{\delta, \theta}$, temos:

se $\operatorname{Re} \lambda>\kappa$, então $d\left(\lambda, \overline{W\left(A_{\varepsilon}\right)}\right) \geq|\lambda-\kappa| \operatorname{sen}(|\arg (\lambda-\kappa)|) \geq|\lambda-\kappa| \operatorname{sen} \theta$;

se $\operatorname{Re} \lambda \leq \kappa$, então $d\left(\lambda, \overline{W\left(A_{\varepsilon}\right)}\right) \geq|\lambda-\kappa| \geq|\lambda-\kappa| \operatorname{sen} \theta$;

o que implica,

$$
\frac{1}{d\left(\lambda, \overline{W\left(A_{\varepsilon}\right)}\right)} \leq \frac{(\operatorname{sen} \theta)^{-1}}{|\lambda-\kappa|}, \forall \lambda \in \Sigma_{\delta, \theta}
$$

Como $\frac{|\lambda|+1}{|\lambda-\kappa|} \rightarrow 1$ quando $|\lambda| \rightarrow+\infty$, existe uma constante $C^{\prime}$ tal que

$$
\frac{1}{|\lambda-\kappa|} \leq \frac{C^{\prime}}{|\lambda|+1}, \forall \lambda \in \Sigma_{\delta, \theta}
$$

Portanto,

$$
\left\|\left(\lambda-A_{\varepsilon}\right)^{-1}\right\| \leq \frac{C}{|\lambda|+1}, \quad \forall \lambda \in \Sigma_{\delta, \theta}
$$

onde $C=C^{\prime}(\operatorname{sen} \theta)^{-1}$. 


\subsection{Convergência do problema aproximado}

Nesta seção mostraremos que, dados $u_{0} \in L^{2}(-1,1)$ e $t_{0} \leq t \leq t_{1}$, a família de soluções $u^{\varepsilon}(t)=T_{\varepsilon}\left(t ; t_{0}\right) u_{0}$ converge para $u^{0}(t)=T_{0}\left(t ; t_{0}\right) u_{0}$ em $L^{2}(-1,1)$ quando $\varepsilon \rightarrow 0$. Para isto, primeiramente, analisamos a convergência do semigrupo linear $\left\{e^{-A_{\varepsilon} t} ; t \geq 0\right\}$.

Lema 2.6 Para cada $t \geq 0$, o semigrupo analítico $e^{-A_{\varepsilon} t} \rightarrow e^{-A_{0} t}$ em $\mathcal{L}(X)$ quando $\varepsilon \rightarrow 0$.

Demonstração. Sabemos de Henry [11] que

$$
e^{-A_{\varepsilon} t}=\frac{1}{2 \pi i} \int_{\Gamma} e^{\lambda t}\left(\lambda+A_{\varepsilon}\right)^{-1} d \lambda, \quad 0 \leq \varepsilon \leq 1
$$

onde $\Gamma$ é a fronteira do setor $\Sigma_{-\delta}=\{\lambda \in \mathbb{C}: 0 \leq|\arg (\lambda+\delta)|<\pi-\theta\}$, que é simétrico em relação ao eixo imaginário ao setor $\Sigma_{\delta, \theta}$, orientada de tal forma que a parte imaginária de $\lambda$ cresce quando $\lambda$ vai percorrendo a curva $\Gamma$. Isto implica que

$$
\left\|e^{-A_{\varepsilon} t}-e^{-A_{0} t}\right\| \leq \frac{1}{2 \pi} \int_{\Gamma} e^{\operatorname{Re} \lambda t}\left\|\left(\lambda+A_{\varepsilon}\right)^{-1}-\left(\lambda+A_{0}\right)^{-1}\right\||d \lambda| .
$$

Segue do Lema 2.5 que, para todo $\eta>0$, existe $R=R(\eta)>0$ tal que

$$
\left\|\left(\lambda+A_{\varepsilon}\right)^{-1}-\left(\lambda+A_{0}\right)^{-1}\right\| \leq \frac{2 C}{1+|\lambda|}<\eta
$$

para todo $\lambda \in \Gamma \operatorname{com}|\lambda|>R$ e $\varepsilon \in[0,1]$. Para este $R=R(\eta)$, temos pelo Lema 2.4, que existe $\varepsilon_{0}>0$ suficientemente pequeno tal que

$$
\left\|\left(\lambda+A_{\varepsilon}\right)^{-1}-\left(\lambda+A_{0}\right)^{-1}\right\|<\eta, \text { para todo } \lambda \in \Gamma \operatorname{com}|\lambda| \leq R \text { e } \varepsilon \in\left[0, \varepsilon_{0}\right] .
$$

Assim, de (2.33) temos

$$
\left\|e^{-A_{\varepsilon} t}-e^{-A_{0} t}\right\| \leq \frac{\eta}{2 \pi} \int_{\Gamma} e^{\operatorname{Re} \lambda t}|d \lambda| \leq \frac{\eta}{2 \pi}\left(\frac{(\sec \theta)^{2} e^{-\delta t}}{t}\right),
$$

para todo $t>0$ e $\varepsilon \in\left[0, \varepsilon_{0}\right]$. Como $\eta>0$ é arbitrário, segue que $e^{-A_{\varepsilon} t} \rightarrow e^{-A_{0} t}$ quando $\varepsilon \rightarrow 0$, para cada $t>0$ fixado.

Teorema 2.3 Sejam $u_{0} \in L^{2}(-1,1)$ e $t_{0} \leq t \leq t_{1}$, então $u^{\varepsilon}(t)=T_{\varepsilon}\left(t ; t_{0}\right) u_{0}$ converge para $u^{0}(t)=T_{0}\left(t ; t_{0}\right) u_{0}$ em $L^{2}(-1,1)$ quando $\varepsilon \rightarrow 0$. 
Demonstração. Da fórmula de variação das constantes temos

$$
u^{\varepsilon}(t)=e^{-A_{\varepsilon}\left(t-t_{0}\right)} u_{0}+\int_{t_{0}}^{t} e^{-A_{\varepsilon}(t-s)} B_{\varepsilon}(s) u^{\varepsilon}(s) d s,
$$

para todo $\varepsilon \in[0,1]$. Então

$$
\begin{aligned}
\left\|u^{\varepsilon}(t)-u^{0}(t)\right\| \leq & \left\|\left(e^{-A_{\varepsilon}\left(t-t_{0}\right)}-e^{-A\left(t-t_{0}\right)}\right) u_{0}\right\|+ \\
& +\int_{t_{0}}^{t}\left\|e^{-A_{\varepsilon}(t-s)} B_{\varepsilon}(s) u^{\varepsilon}(s)-e^{-A_{0}(t-s)} B_{0}(s) u^{0}(s)\right\| d s .
\end{aligned}
$$

Por sua vez,

$$
\begin{aligned}
e^{-A_{\varepsilon}(t-s)} B_{\varepsilon}(s) u^{\varepsilon}(s)-e^{-A_{0}(t-s)} B_{0}(s) u^{0}(s)= & e^{-A_{\varepsilon}(t-s)} B_{\varepsilon}(s)\left[u^{\varepsilon}(s)-u^{0}(s)\right]+ \\
& +e^{-A_{\varepsilon}(t-s)}\left[B_{\varepsilon}(s)-B_{0}(s)\right] u^{0}(s)+ \\
& +\left[e^{-A_{\varepsilon}(t-s)}-e^{-A_{0}(t-s)}\right] B_{0}(s) u^{0}(s)
\end{aligned}
$$

o que implica

$$
\begin{aligned}
\left\|u^{\varepsilon}(t)-u^{0}(t)\right\| \leq & \left\|\left(e^{-A_{\varepsilon}\left(t-t_{0}\right)}-e^{-A\left(t-t_{0}\right)}\right) u_{0}\right\|+\int_{t_{0}}^{t}\left\|e^{-A_{\varepsilon}(t-s)}\right\|\left\|\left(B_{\varepsilon}(s)-B_{0}(s)\right) u^{0}(s)\right\| d s \\
& +\int_{t_{0}}^{t}\left\|e^{-A_{\varepsilon}(t-s)}-e^{-A_{0}(t-s)}\right\|\left\|B_{0}(s) u^{0}(s)\right\| d s+ \\
& +\int_{t_{0}}^{t}\left\|e^{-A_{\varepsilon}(t-s)}\right\|\left\|B_{\varepsilon}(s)\right\|\left\|u^{\varepsilon}(s)-u^{0}(s)\right\| d s .
\end{aligned}
$$

Segue do Lema 2.5 e da limitação de $\left\{\left\|B_{\varepsilon}(t)\right\| ; t_{0} \leq t \leq t_{1}, \varepsilon \in[0,1]\right\}$ que

$$
\begin{aligned}
\left\|u^{\varepsilon}(t)-u^{0}(t)\right\| \leq & \left\|\left(e^{-A_{\varepsilon}\left(t-t_{0}\right)}-e^{-A\left(t-t_{0}\right)}\right) u_{0}\right\|+\int_{t_{0}}^{t} e^{-\delta(t-s)}\left\|\left(B_{\varepsilon}(s)-B_{0}(s)\right) u^{0}(s)\right\| d s+ \\
& +\int_{t_{0}}^{t}\left\|e^{-A_{\varepsilon}(t-s)}-e^{-A_{0}(t-s)}\right\|\left\|B_{0}(s) u^{0}(s)\right\| d s+ \\
& +K \int_{t_{0}}^{t} e^{-\delta(t-s)}\left\|u^{\varepsilon}(s)-u^{0}(s)\right\| d s .
\end{aligned}
$$

Escrevendo de forma equivalente temos

$$
\begin{aligned}
e^{\delta\left(t-t_{0}\right)}\left\|u^{\varepsilon}(t)-u^{0}(t)\right\| & \leq e^{\delta\left(t-t_{0}\right)}\left\|\left(e^{-A_{\varepsilon}\left(t-t_{0}\right)}-e^{-A\left(t-t_{0}\right)}\right) u_{0}\right\|+ \\
& +\int_{t_{0}}^{t} e^{\delta\left(s-t_{0}\right)}\left\|\left(B_{\varepsilon}(s)-B_{0}(s)\right) u^{0}(s)\right\| d s+ \\
& +e^{\delta\left(t-t_{0}\right)} \int_{t_{0}}^{t}\left\|e^{-A_{\varepsilon}(t-s)}-e^{-A_{0}(t-s)}\right\|\left\|B_{0}(s) u^{0}(s)\right\| d s+ \\
& +K \int_{t_{0}}^{t} e^{\delta\left(s-t_{0}\right)}\left\|u^{\varepsilon}(s)-u^{0}(s)\right\| d s
\end{aligned}
$$


Do Teorema da Convergência Dominada e do Lema 2.6, para cada $t_{0} \leq t \leq t_{1}$, temos

$$
e^{\delta\left(t-t_{0}\right)}\left\|u^{\varepsilon}(t)-u^{0}(t)\right\| \leq \mathrm{o}(1)+K \int_{t_{0}}^{t} e^{\delta\left(s-t_{0}\right)} \|\left(u^{\varepsilon}(s)-u^{0}(s) \| d s,\right.
$$

quando $\varepsilon \rightarrow 0$. Aplicando a desigualdade de Gronwall, concluímos que

$$
\left\|u^{\varepsilon}(t)-u^{0}(t)\right\| \leq \mathrm{o}(1) e^{(K-\delta)\left(t-t_{0}\right)}
$$

quando $\varepsilon \rightarrow 0$. Portanto, $u^{\varepsilon}(t) \rightarrow u^{0}(t)$ em $L^{2}(-1,1)$ quando $\varepsilon \rightarrow 0$, uniformemente para $t_{0} \leq t \leq t_{1}$.

Como consequência imediata do Teorema 2.1, temos o seguinte corolário.

Corolário 2.1 Para $0<\varepsilon \leq 1$, seja $u^{\varepsilon}(t)=T_{\varepsilon}\left(t ; t_{0}\right) u^{\varepsilon}\left(t_{0}\right)$ uma solução clássica do problema

$$
\left\{\begin{array}{l}
u_{t}^{\varepsilon}=\left(a_{\varepsilon}(x) u_{x}^{\varepsilon}\right)_{x}+b_{\varepsilon}(x, t) u^{\varepsilon}, x \in(-1,1), t_{0}<t \leq t_{1} \\
u^{\varepsilon}(-1, t)=u^{\varepsilon}(1, t)=0
\end{array}\right.
$$

onde $a_{\varepsilon}$ e $b_{\varepsilon}$ satisfazem as hipóteses da seção anterior. Então $\mathcal{S}\left(u^{\varepsilon}\left(t_{1}\right)\right) \leq \mathcal{S}\left(u^{\varepsilon}\left(t_{0}\right)\right)$.

Finalmente, podemos demonstrar o resultado principal deste capítulo.

Teorema 2.4 Seja $a_{0}$ uma função positiva e limitada de classe $C^{1}$ em $[-1,0) \cup(0,1]$ e $b_{0}$ uma função contínua e limitada em $([-1,0) \cup(0,1]) \times\left[t_{0}, t_{1}\right]$. Seja u uma solução clássica do problema,

$$
\left\{\begin{array}{l}
u_{t}=\left(a_{0}(x) u_{x}\right)_{x}+b_{0}(x, t) u, x \in(-1,0) \cup(0,1), t_{0}<t \leq t_{1} \\
u(0-, t)=u(0+, t) \\
a_{0}(0-) u_{x}(0-, t)=a_{0}(0+) u_{x}(0+, t) \\
u(-1, t)=u(1, t)=0
\end{array}\right.
$$

isto é, u e $a_{0} u_{x}$ são contínuas em $[-1,1] \times\left[t_{0}, t_{1}\right]$ e $[-1,1] \times\left(t_{0}, t_{1}\right]$, respectivamente, $u_{t}$ e $u_{x x}$ são contínuas em $((-1,0) \cup(0,1)) \times\left(t_{0}, t_{1}\right]$. Se $\mathcal{S}\left(u\left(t_{1}\right)\right)<\infty$ então

$$
\mathcal{S}\left(u\left(t_{1}\right)\right) \leq \mathcal{S}\left(u\left(t_{0}\right)\right)
$$

Demonstração. Considere $u^{\varepsilon}(t)=T_{\varepsilon}\left(t, t_{0}\right) u\left(t_{0}\right)$ a solução do problema (2.35) que passa por $u\left(t_{0}\right)$ no instante $t=t_{0}$. Segue do Teorema 2.3 que

$$
u^{\varepsilon}\left(t_{1}\right) \rightarrow u\left(t_{1}\right) \text { em } L^{2}(-1,1) \text { quando } \varepsilon \rightarrow 0 .
$$


Como por hipótese $\mathcal{S}\left(u\left(t_{1}\right)\right)<\infty$, segue de (2.37) que $\mathcal{S}\left(u^{\varepsilon}\left(t_{1}\right)\right) \geq \mathcal{S}\left(u\left(t_{1}\right)\right)$, para todo $\varepsilon>0$ suficientemente pequeno. Como $u^{\varepsilon}\left(t_{0}\right)=T_{\varepsilon}\left(t_{0}, t_{0}\right) u\left(t_{0}\right)=u\left(t_{0}\right)$, para todo $\varepsilon>0$, temos do Corolário 2.1 que

$$
\mathcal{S}\left(u\left(t_{0}\right)\right)=\mathcal{S}\left(u^{\varepsilon}\left(t_{0}\right)\right) \geq \mathcal{S}\left(u^{\varepsilon}\left(t_{1}\right)\right) \geq \mathcal{S}\left(u\left(t_{1}\right)\right),
$$

como queríamos demonstrar. 


\section{Capítulo 3}

\section{Transversalidade das Variedades}

\section{Estável e Instável}

Neste capítulo mostraremos que as variedades estável e instável de pontos de equilíbrio hiperbólicos de $(1.1)$ se interseptam transversalmente. Seja $\bar{w}(t)=(\bar{u}(t), \bar{v}(t))$ uma solução não constante de (1.1) definida em $-\infty<t<+\infty$ tal que

$$
\bar{w}(t) \longrightarrow \varphi_{ \pm} \text {em } H \text { quando } t \rightarrow \pm \infty
$$

onde $\varphi_{ \pm}$são pontos de equilíbrio hiperbólicos de (1.1).

Lema 3.1 Sejam $\bar{w}$ e $\varphi_{ \pm}$como acima. Então a convergência em (3.1) é exponencial e $\bar{w}_{t}(t) \rightarrow 0$ exponencialmente em $H$ quando $t \rightarrow \pm \infty$.

Demonstração. De fato, escrevendo $\bar{w}(t)=\varphi_{ \pm}+\bar{z}(t)$ segue que $\bar{z}(t) \rightarrow 0$ em $H$ quando $t \rightarrow \pm \infty$ e $\bar{z}(t)$ é uma solução do problema

$$
z_{t}=-\left(A-N^{\prime}\left(\varphi_{ \pm}\right)\right) z+R(z)
$$

onde $R$ é dada por $R(z)=N\left(\varphi_{ \pm}+z\right)-N\left(\varphi_{ \pm}\right)-N^{\prime}\left(\varphi_{ \pm}\right) z$, isto é, $R$ é uma aplicação de $X^{1 / 2}=H$ em $X$, de classe $C^{1}, R(0)=0, R^{\prime}(0)=0$ e $\|R(z)\|=o\left(\|z\|_{\alpha}\right)$ quando $\|z\|_{\alpha} \rightarrow 0$. Denotando o operador $L_{ \pm}=-A+N^{\prime}\left(\varphi_{ \pm}\right)$, reescrevemos (3.2) como

$$
z_{t}=L_{ \pm} z+R(z)
$$

Como $\varphi_{ \pm}$é um ponto de equilíbrio hiperbólico, segue que o espectro do operador $L_{ \pm}$ é disjunto do eixo imaginário. Além disso, $-L_{ \pm}$é setorial então, podemos separar seu 
espectro em dois conjuntos espectrais $\sigma_{1}$ e $\sigma_{2}$, onde $\sigma_{1}=\sigma\left(-L_{ \pm}\right) \cap\{\lambda: R e \lambda<0\}$ é limitado, no nosso caso é formado por um número finito de autovalores, e $\sigma_{2}=\sigma\left(-L_{ \pm}\right) \cap$ $\{\lambda: \operatorname{Re} \lambda>0\}$. Considere as projeções $E_{1}$ e $E_{2}$ associadas aos conjuntos espectrais $\sigma_{1}$ e $\sigma_{2}$, e sejam $X_{j}=E_{j}(X), j=1,2$. Segue do Teorema 1.5.2 [11] que $X=X_{1} \oplus X_{2}, X_{j}$ são invariantes por $L_{ \pm}$e, se $L_{ \pm}^{j}=\left.L_{ \pm}\right|_{X_{j}}$, então

$$
\begin{gathered}
-L_{ \pm}^{1}: X_{1} \rightarrow X_{1} \text { é um operador linear limitado, } \sigma\left(-L_{ \pm}^{1}\right)=\sigma_{1} \\
-L_{ \pm}^{2}: D\left(L_{ \pm}\right) \cap X_{2} \rightarrow X_{2} \text { é um operador setorial e } \sigma\left(-L_{ \pm}^{2}\right)=\sigma_{2} .
\end{gathered}
$$

Vamos considerar o caso $t \rightarrow+\infty$; o caso $t \rightarrow-\infty$ acreditamos ser análogo. Como $\bar{z}(t) \rightarrow 0$ em $H$ quando $t \rightarrow+\infty$, temos que existe $t_{0}>0$, suficientemente grande, tal que $\bar{z}(t) \in W_{\text {loc }}^{s}(0)$, para todo $t \geq t_{0}$, isto é, $\left\|E_{2} \bar{z}\left(t_{0}\right)\right\|_{\alpha} \leq \frac{\rho}{2 M}$ e $\|\bar{z}(t)\|_{\alpha} \leq \rho$, para todo $t \geq t_{0}$. Mais ainda, na demonstração do Teorema 1.2, temos que $W_{\text {loc }}^{s}$ é dada como sendo o gráfico de uma aplicação $h$ de classe $C^{1}$ definida na bola de centro 0 e raio $\frac{\rho}{2 M}$ em $X_{2}^{\alpha}$ com valores em $X_{1}$, tal que $h(0)=0$ e $h^{\prime}(0)=0$. Assim, se $\bar{z}(t)=\bar{z}_{1}(t)+\bar{z}_{2}(t) \in X_{1} \oplus X_{2}$, segue que $\bar{z}_{1}(t)=h\left(\bar{z}_{2}(t)\right)$, para todo $t \geq t_{0}$.

Da fórmula da variação das constantes temos que

$$
\bar{z}_{2}(t)=e^{L_{+}^{2}\left(t-t_{0}\right)} E_{2} \bar{z}\left(t_{0}\right)+\int_{t_{0}}^{t} e^{L_{+}^{2}(t-s)} E_{2} R(\bar{z}(s)) d s,
$$

para todo $t \geq t_{0}$. Sejam $\beta$ e $\beta^{\prime}$ tais que $0<\beta<\beta^{\prime}<\operatorname{Re} \sigma\left(-\mathrm{L}_{ \pm}^{2}\right)$,

$$
\left\|e^{t L_{+}^{2}} z\right\|_{\alpha} \leq M e^{-\beta^{\prime} t}\|z\|_{\alpha} \quad \text { e }\left\|e^{t L_{+}^{2}} z\right\|_{\alpha} \leq M t^{-\alpha} e^{-\beta^{\prime} t}\|z\| .
$$

Consideremos $\eta>0$, suficientemente pequeno, de modo que

$$
2 \eta M\left\|E_{2}\right\| \int_{0}^{+\infty} s^{-\alpha} e^{-\left(\beta^{\prime}-\beta\right) s} d s<1 / 2,
$$

e diminuimos $\rho>0$, caso necessário, de forma que $\|R(z)\| \leq \eta\|z\|_{\alpha}$, sempre que $\|z\|_{\alpha} \leq \rho$. Assim,

$$
\begin{aligned}
\left\|\bar{z}_{2}(t)\right\|_{\alpha} & \leq M e^{-\beta\left(t-t_{0}\right)}\left\|E_{2} \bar{z}\left(t_{0}\right)\right\|_{\alpha}+\int_{t_{0}}^{t} M(t-s)^{-\alpha} e^{-\beta^{\prime}(t-s)}\left\|E_{2}\right\|\|R(\bar{z}(s))\| d s \\
& \leq M e^{-\beta\left(t-t_{0}\right)}\left\|E_{2} \bar{z}\left(t_{0}\right)\right\|_{\alpha}+\eta M\left\|E_{2}\right\| \int_{t_{0}}^{t}(t-s)^{-\alpha} e^{-\beta^{\prime}(t-s)}\|\bar{z}(s)\|_{\alpha} d s
\end{aligned}
$$

para todo $t \geq t_{0}$. Com as hipóteses para $h$, podemos concluir, aumentando $t_{0}$ se necessário, que

$$
\|\bar{z}(s)\|_{\alpha}=\left\|h\left(\bar{z}_{2}(s)\right)\right\|_{\alpha}+\left\|\bar{z}_{2}(s)\right\|_{\alpha} \leq 2\left\|\bar{z}_{2}(s)\right\|_{\alpha}
$$


para todo $s \geq t_{0}$. Substituindo na inequação,

$$
\begin{aligned}
\left\|\bar{z}_{2}(t)\right\|_{\alpha} & \leq M e^{-\beta\left(t-t_{0}\right)}\left\|E_{2} \bar{z}\left(t_{0}\right)\right\|_{\alpha}+2 \eta M\left\|E_{2}\right\| \int_{t_{0}}^{t}(t-s)^{-\alpha} e^{-\beta^{\prime}(t-s)}\left\|\bar{z}_{2}(s)\right\|_{\alpha} d s \\
e^{\beta\left(t-t_{0}\right)}\left\|\bar{z}_{2}(t)\right\|_{\alpha} & \leq M\left\|E_{2} \bar{z}\left(t_{0}\right)\right\|_{\alpha}+2 \eta M\left\|E_{2}\right\| \int_{t_{0}}^{t}(t-s)^{-\alpha} e^{-\left(\beta^{\prime}-\beta\right)(t-s)}\left\|\bar{z}_{2}(s)\right\|_{\alpha} d s,
\end{aligned}
$$

para todo $t \geq t_{0}$. Se $y(s)=\sup \left\{e^{\beta\left(s-t_{0}\right)}\left\|\bar{z}_{2}(s)\right\|_{\alpha} ; t_{0} \leq s \leq t\right\}$ então

$$
\begin{aligned}
e^{\beta\left(t-t_{0}\right)}\left\|\bar{z}_{2}(t)\right\|_{\alpha} & \leq M\left\|E_{2} \bar{z}\left(t_{0}\right)\right\|_{\alpha}+\left(2 \eta M\left\|E_{2}\right\| \int_{t_{0}}^{t}(t-s)^{-\alpha} e^{-\left(\beta^{\prime}-\beta\right)(t-s)} d s\right) y(t) \\
& \leq M\left\|E_{2} \bar{z}\left(t_{0}\right)\right\|_{\alpha}+\frac{1}{2} y(t)
\end{aligned}
$$

para todo $t \geq t_{0}$. Portanto, $y(t) \leq 2 M\left\|E_{2} \bar{z}\left(t_{0}\right)\right\|_{\alpha}$, para todo $t \geq t_{0}$, o que implica

$$
\left\|\bar{z}_{2}(t)\right\|_{\alpha} \leq 2 M\left\|E_{2} \bar{z}\left(t_{0}\right)\right\|_{\alpha} e^{-\beta\left(t-t_{0}\right)} \leq \rho e^{-\beta\left(t-t_{0}\right)}, \text { para todo } t \geq t_{0}
$$

De (3.4) e (3.5) segue que $\|\bar{z}(t)\|_{\alpha} \leq 2 \rho e^{-\beta\left(t-t_{0}\right)}$, para todo $t \geq t_{0}$, isto é,

$$
\left\|\bar{w}(t)-\varphi_{+}\right\|_{\alpha} \leq 2 \rho e^{-\beta\left(t-t_{0}\right)}, \text { para todo } t \geq t_{0} .
$$

Para mostrarmos a segunda parte do lema, linearizamos o problema (0.1)-(0.3) em torno da solução $\bar{w}(t)=(\bar{u}(t), \bar{v}(t))$,

$$
\left\{\begin{array}{l}
u_{t}=\left(a(x) u_{x}\right)_{x}+c(x, t) u, x \in(0,1), \quad t \in(-\infty,+\infty) \\
v_{t}=\left(b(x) v_{x}\right)_{x}+d(x, t) v, \quad x \in(0,1) \\
u(0, t)=v(0, t) \\
a(0) u_{x}(0, t)+b(0) v_{x}(0, t)=0 \\
u(1, t)=v(1, t)=0
\end{array}\right.
$$

onde $c(x, t)=f^{\prime}(\bar{u}(x, t))$ e $d(x, t)=g^{\prime}(\bar{v}(x, t))$ para $0 \leq x \leq 1$ e $-\infty<t<+\infty$.

Observe que $\bar{w}_{t}(\cdot, t)=\left(\bar{u}_{t}(\cdot, t), \bar{v}_{t}(\cdot, t)\right)$ é uma solução não nula de (3.6). Além disso, se $\bar{w}(t)=\varphi_{ \pm}+\bar{z}(t)$, então $\bar{w}_{t}(t)=\bar{z}_{t}(t)$ e $\bar{z}_{t}(t)$ é uma solução da seguinte equação de evolução

$$
\theta_{t}=L_{ \pm} \theta+R^{\prime}(\bar{z}(t)) \theta
$$

Denotemos $\bar{\theta}(t)=\bar{z}_{t}(t)=\bar{\theta}_{1}(t)+\bar{\theta}_{2}(t) \in X_{1} \oplus X_{2}$. Como $\bar{z}_{1}(t)=h\left(\bar{z}_{2}(t)\right)$, para todo $t \geq t_{0}$, temos que $\bar{\theta}_{1}(t)=h^{\prime}\left(\bar{z}_{2}(t)\right) \bar{\theta}_{2}(t)$, para todo $t \geq t_{0}$. Da fórmula da variação das constantes temos

$$
\bar{\theta}_{2}(t)=e^{L_{+}^{2}\left(t-t_{0}\right)} E_{2} \bar{\theta}\left(t_{0}\right)+\int_{t_{0}}^{t} e^{L_{+}^{2}(t-s)} E_{2} R^{\prime}(\bar{z}(s)) \bar{\theta}(s) d s,
$$


para todo $t \geq t_{0}$. Como $R$ é de classe $C^{1}$ e $R^{\prime}(0)=0$, aumentando $t_{0}$ se necessário, temos que $\left\|R^{\prime}(\bar{z}(t))\right\| \leq \eta$, para todo $t \geq t_{0}$.

Por sua vez, $\left.\|\bar{\theta}(t)\|_{\alpha}=\| h^{\prime}\left(\bar{z}_{2}(t)\right) \theta_{2} \overline{(} t\right)\left\|_{\alpha}+\right\| \bar{\theta}_{2}(t) \|_{\alpha}$, para todo $t \geq t_{0}$, e h é de classe $C^{1}$ com $h^{\prime}(0)=0$, então, aumentando novamente $t_{0}$ temos $\|\bar{\theta}(t)\|_{\alpha} \leq 2\left\|\bar{\theta}_{2}(t)\right\|_{\alpha}$, para todo $t \geq t_{0}$. Assim,

$$
\begin{aligned}
\left\|\bar{\theta}_{2}(t)\right\|_{\alpha} & \leq M e^{-\beta\left(t-t_{0}\right)}\left\|E_{2} \bar{\theta}\left(t_{0}\right)\right\|_{\alpha}+\eta M \int_{t_{0}}^{t}(t-s)^{-\alpha} e^{-\beta^{\prime}(t-s)}\left\|E_{2}\right\|\|\bar{\theta}(s)\|_{\alpha} d s \\
& \leq M e^{-\beta\left(t-t_{0}\right)}\left\|E_{2} \bar{\theta}\left(t_{0}\right)\right\|_{\alpha}+2 \eta M\left\|E_{2}\right\| \int_{t_{0}}^{t}(t-s)^{-\alpha} e^{-\beta^{\prime}(t-s)}\left\|\bar{\theta}_{2}(s)\right\|_{\alpha} d s
\end{aligned}
$$

para todo $t \geq t_{0}$. Da mesma forma que o caso $\bar{z}_{2}(t)$, podemos concluir que

$$
\left\|\bar{\theta}_{2}(t)\right\|_{\alpha} \leq 2 M\left\|E_{2} \bar{\theta}\left(t_{0}\right)\right\|_{\alpha} e^{-\beta\left(t-t_{0}\right)},
$$

para todo $t \geq t_{0}$. Portanto,

$$
\left\|\bar{w}_{t}(t)\right\|_{\alpha}=\left\|\bar{z}_{t}(t)\right\|_{\alpha} \leq 2\left\|\bar{\theta}_{2}(t)\right\|_{\alpha} \leq K e^{-\beta\left(t-t_{0}\right)}
$$

para todo $t \geq t_{0}$, onde $K=4 M\left\|E_{2} \bar{\theta}\left(t_{0}\right)\right\|_{\alpha}$.

Vamos provar que as variedades instável de $\varphi_{-}$e estável de $\varphi_{+}$se interseptam transversalmente em $\bar{w}(t)$, para cada $t$, isto é,

$$
W^{u}\left(\varphi_{-}\right) \pitchfork_{\bar{w}(t)} W^{s}\left(\varphi_{+}\right)
$$

De (1.10) e (1.11), segue que os espaços tangentes às variedades $W^{u}\left(\varphi_{-}\right)$e $W^{s}\left(\varphi_{+}\right)$em $\bar{w}\left(t_{0}\right)$ são dados por

$$
\begin{aligned}
T_{\bar{w}\left(t_{0}\right)} W^{s}\left(\varphi_{+}\right)= & \left\{w\left(t_{0}\right) \in H \text { tal que } w(t) \text { é uma solução de }(3.6) \text { em } t_{0}<t<+\infty\right. \\
& \text { e } \left.\|w(t)\|_{X}=O\left(e^{-\epsilon t}\right) \text { quando } t \rightarrow+\infty \text {, para algum } \epsilon>0\right\}, \\
T_{\bar{w}\left(t_{0}\right)} W^{u}\left(\varphi_{-}\right)= & \left\{w\left(t_{0}\right) \in H \text { tal que } w(t) \text { é uma solução de }(3.6) \text { em }-\infty<t<t_{0}\right. \\
& \text { e } \left.\|w(t)\|_{X}=O\left(e^{\epsilon t}\right) \text { quando } t \rightarrow-\infty, \text { para algum } \epsilon>0\right\} .
\end{aligned}
$$

Note que $\bar{w}_{t}\left(t_{0}\right) \in T_{\bar{w}\left(t_{0}\right)} W^{u}\left(\varphi_{-}\right) \cap T_{\bar{w}\left(t_{0}\right)} W^{s}\left(\varphi_{+}\right)$.

O próximo resultado é uma caracterização de $T_{\bar{w}\left(t_{0}\right)} W^{s}\left(\varphi_{+}\right)$e $T_{\bar{w}\left(t_{0}\right)} W^{u}\left(\varphi_{-}\right)$, onde serão utilizadas as soluções $\left\{w_{j}^{-}(t): j \geq 1\right\}$ obtidas no Teorema 1.7 e as soluções $\left\{\eta_{k}^{+}(t)\right.$ : 
$k \geq 1$ \} obtidas no Corolário 1.5. Com o Lema 3.1, não é difícil mostrar que as hipóteses do Teorema 1.7 estão satisfeitas para as funções $c(x, t)=f^{\prime}(\bar{u}(x, t))$ e $d(x, t)=g^{\prime}(\bar{v}(x, t))$, $0 \leq x \leq 1$ e $-\infty<t<+\infty$.

Teorema 3.1 Suponha que $\lambda_{m}^{+}$é o m-ésimo autovalor da linearização de $(0.1)-(0.3)$ em $\varphi_{+}=\left(\varphi_{+}^{1}, \varphi_{+}^{2}\right)$ com $\lambda_{m}^{+}>0>\lambda_{m+1}^{+}$, e $\lambda_{n}^{-}$é o n-ésimo autovalor da linearização de $(0.1)-(0.3)$ em $\varphi_{-}=\left(\varphi_{-}^{1}, \varphi_{-}^{2}\right) \operatorname{com} \lambda_{n}^{-}>0>\lambda_{n+1}^{-}$. Então,

$$
T_{\bar{w}\left(t_{0}\right)} W^{s}\left(\varphi_{+}\right)=\left\{\psi \in H:\left\langle\psi, \eta_{k}^{+}\left(t_{0}\right)\right\rangle_{X}=0, \text { para } 1 \leq k \leq m\right\}
$$

$e$

$$
T_{\bar{w}\left(t_{0}\right)} W^{u}\left(\varphi_{-}\right)=\text {subespaço gerado por }\left\{w_{j}^{-}\left(t_{0}\right): 1 \leq j \leq n\right\}
$$

Demonstração. Seja $U:=\left\{\psi \in H:\left\langle\psi, \eta_{k}^{+}\left(t_{0}\right)\right\rangle_{X}=0\right.$, para $\left.1 \leq k \leq m\right\}$. Se $\psi \in T_{\bar{w}\left(t_{0}\right)} W^{s}\left(\varphi_{+}\right)$então $\psi=w\left(t_{0}\right)$, onde $w(t)$ resolve (3.6) para $t_{0}<t<+\infty$ e $\|w(t)\|_{X}=O\left(e^{-\epsilon t}\right)$ quando $t \rightarrow+\infty$, para algum $\epsilon>0$. Assim, segue do Teorema 1.8 que existem inteiro $j \geq 1$ e constante $C \neq 0$ tais que

$$
w(t)=\exp \left(\int_{0}^{t} \lambda_{j}(s) d s\right)\left[C \psi_{j}^{+}+o(1)\right] \text { quando } t \rightarrow+\infty
$$

Como $\lambda_{j}(t) \rightarrow \lambda_{j}^{+}$e $\|w(t)\|_{X}=O\left(e^{-\epsilon t}\right)$ quando $t \rightarrow+\infty$, temos $j>m$.

Para $1 \leq k \leq m$, considere as soluções do problema adjunto $\eta_{k}^{+}$dadas pelo Corolário 1.5 e definidas para $-\infty<t<+\infty$. Segue da Observação 1.1 que

$$
\begin{aligned}
\left\langle\psi, \eta_{k}^{+}\left(t_{0}\right)\right\rangle_{X} & =\left\langle w(t), \eta_{k}^{+}(t)\right\rangle_{X} \\
& =\left\langle\exp \left(\int_{0}^{t} \lambda_{j}(s) d s\right)\left[C \psi_{j}^{+}+o(1)\right], \exp \left(-\int_{0}^{t} \lambda_{k}(s) d s\right)\left[\psi_{k}^{+}+o(1)\right]\right\rangle_{X} \\
& =\exp \left(\int_{0}^{t}\left[\lambda_{j}(s)-\lambda_{k}(s)\right] d s\right)\left[C\left\langle\psi_{j}^{+}, \psi_{k}^{+}\right\rangle_{X}+o(1)\right] \\
& =\exp \left(\int_{0}^{t}\left[\lambda_{j}(s)-\lambda_{k}(s)\right] d s\right) o(1)
\end{aligned}
$$

quando $t \rightarrow+\infty$, para todo $1 \leq k \leq m$, pois $k \leq m<j$. Como $0>\lambda_{j}(t)-\lambda_{k}(t) \rightarrow$ $\lambda_{j}^{+}-\lambda_{k}^{+}<0$ quando $t \rightarrow+\infty$, segue que $\left\langle\psi, \eta_{k}^{+}\left(t_{0}\right)\right\rangle_{X}=0$, para todo $k=1, \cdots, m$, isto é, $\psi \in U$. Portanto, $T_{\bar{w}\left(t_{0}\right)} W^{s}\left(\varphi_{+}\right) \subset U$.

Reciprocamente, se $\psi \in U$, seja $w(t)$ a solução de (3.6) com $t>t_{0}$ tal que $w\left(t_{0}\right)=\psi$. Novamente, usando o Teorema 1.8 e o Corolário 1.5 , existem um inteiro $j \geq 1$ e uma 
constante $C \neq 0$ tais que, para todo $k=1, \cdots, m$,

$$
\begin{aligned}
0 & =\left\langle\psi, \eta_{k}^{+}\left(t_{0}\right)\right\rangle_{X}=\left\langle w(t), \eta_{k}^{+}(t)\right\rangle_{X} \\
& =\left\langle\exp \left(\int_{0}^{t} \lambda_{j}(s) d s\right)\left[C \psi_{j}^{+}+o(1)\right], \exp \left(-\int_{0}^{t} \lambda_{k}(s) d s\right)\left[\psi_{k}^{+}+o(1)\right]\right\rangle_{X} \\
& =\exp \left(\int_{0}^{t}\left[\lambda_{j}(s)-\lambda_{k}(s)\right] d s\right)\left[C\left\langle\psi_{j}^{+}, \psi_{k}^{+}\right\rangle_{X}+o(1)\right],
\end{aligned}
$$

quando $t \rightarrow+\infty$.

Se $j \leq m$ então, para $k=j$, obtemos que $0=C+o(1)$, o que é um absurdo visto que $C \neq 0$. Portanto $j>m$, o que implica, $\lambda_{j}^{+}<0$.

Tomando $\epsilon>0$, suficientemente pequeno, de modo que $\lambda_{j}^{+}+\epsilon<0$, segue que

$$
\|w(t)\|_{X}=O\left(e^{\left(\lambda_{j}^{+}+\epsilon\right) t}\right) \text { quando } t \rightarrow+\infty
$$

mostrando assim, que $\psi \in T_{\bar{w}\left(t_{0}\right)} W^{s}\left(\varphi_{+}\right)$. Logo, $U=T_{\bar{w}\left(t_{0}\right)} W^{s}\left(\varphi_{+}\right)$.

Para caracterizar $T_{\bar{w}\left(t_{0}\right)} W^{u}\left(\varphi_{-}\right)$, seja $V:=\left[w_{j}^{-}\left(t_{0}\right): 1 \leq j \leq n\right]$. Se $\psi \in T_{\bar{w}\left(t_{0}\right)} W^{u}\left(\varphi_{-}\right)$, então $\psi=w\left(t_{0}\right)$, onde $w(t)$ resolve (3.6) em $-\infty<t<t_{0}$ e $\|w(t)\|_{X}=O\left(e^{\epsilon t}\right)$ quando $t \rightarrow-\infty$, para algum $\epsilon>0$. Então pelo Teorema 1.9, com $k=-\epsilon$, existem constantes $C_{1}, \cdots, C_{n}$ tais que

$$
w(t)=\sum_{j=1}^{n} C_{j} w_{j}^{-}(t)
$$

e portanto, $\psi=w\left(t_{0}\right) \in V$.

Reciprocamente, se $\psi \in V$, existem constantes $\alpha_{1}, \cdots, \alpha_{n}$ tais que $\psi=\sum_{j=1}^{n} \alpha_{j} w_{j}^{-}\left(t_{0}\right)$. Dessa forma, a função $w(t)=\sum_{j=1}^{n} \alpha_{j} w_{j}^{-}(t)$ resolve (3.6) para $-\infty<t<t_{0}$. Tome $\epsilon>0$ suficientemente pequeno de modo que $\lambda_{n}^{-}-\frac{3}{2} \epsilon>0$ e $T$ suficientemente grande de modo que $\lambda_{j}^{-}-\lambda_{j}(s)<\epsilon / 2$, para todo $s \leq-T$ e para todo $j=1, \cdots, n$. Usando a expressão de $w_{j}^{-}$dada pelo Teorema 1.7 , obtemos

$$
\begin{aligned}
\frac{\|w(t)\|_{X}}{e^{\epsilon t}} & \leq \sum_{j=1}^{n} e^{-\int_{t}^{0}\left(\lambda_{j}(s)-\epsilon\right) d s}\left[\left|\alpha_{j}\right|\left\|\psi_{j}^{-}\right\|_{X}+o(1)\right] \\
& =\sum_{j=1}^{n} e^{\int_{-T}^{0}\left(\epsilon-\lambda_{j}(s)\right) d s} e^{\int_{t}^{-T}\left(\epsilon-\lambda_{j}(s)\right) d s}\left[\left|\alpha_{j}\right|+o(1)\right] \\
& =\sum_{j=1}^{n} e^{\int_{-T}^{0}\left(\epsilon-\lambda_{j}(s)\right) d s} e^{\int_{t}^{-T}\left(\epsilon-\lambda_{j}^{-}\right) d s} e^{\int_{t}^{-T}\left(\lambda_{j}^{-}-\lambda_{j}(s)\right) d s}\left[\left|\alpha_{j}\right|+o(1)\right] \\
& \leq \sum_{j=1}^{n} e^{\int_{-T}^{0}\left(\epsilon-\lambda_{j}(s)\right) d s} e^{\left(\lambda_{j}^{-}-\frac{3}{2} \epsilon\right)(T+t)}\left[\left|\alpha_{j}\right|+o(1)\right] \leq K
\end{aligned}
$$


para qualquer $K>0$, quando $t \rightarrow-\infty$, já que, para todo $1 \leq j \leq n, 0<\lambda_{n}^{-}-\frac{3}{2} \epsilon \leq$ $\lambda_{j}^{-}-\frac{3}{2} \epsilon$. Portanto, $\|w(t)\|_{X}=O\left(e^{\epsilon t}\right)$ quando $t \rightarrow-\infty$, isto é, $\psi \in T_{\bar{w}\left(t_{0}\right)} W^{u}\left(\varphi_{-}\right)$. Logo, $V=T_{\bar{w}\left(t_{0}\right)} W^{u}\left(\varphi_{-}\right)$.

Na demosntração do próximo teorema, utilizaremos o seguinte resultado de Análise Funcional: sejam $\zeta_{1}, \cdots, \zeta_{n}$ funcionais lineares sobre um espaço vetorial normado $E$ e $v_{1}, \cdots, v_{m}$ elementos de $E$. Se a soma do subespaço $\left[v_{1}, \cdots, v_{m}\right]$ com o subespaço $\left\{v \in E: \zeta_{k}(v)=0,1 \leq k \leq n\right\}$ não é todo o $E$, existe um funcional não nulo $\zeta$ no subespaço $\left[\zeta_{1}, \cdots, \zeta_{n}\right]$ que anula todo $v_{j}, j=1, \cdots, m$.

Lema 3.2 Sejam $n_{-}$e $m_{+}$o número de autovalores positivos da linearização em $\varphi_{-} e$ $\varphi_{+}$, respectivamente. Se $n_{-} \geq m_{+} \geq 0$ então

$$
T_{\bar{w}\left(t_{0}\right)} W^{u}\left(\varphi_{-}\right) \pitchfork T_{\bar{w}\left(t_{0}\right)} W^{s}\left(\varphi_{+}\right)
$$

ou ainda, a soma destes subespaços é o espaço inteiro $H$.

Reciprocamente, $n_{-} \geq m_{+}$é uma condição necessária para a transversalidade.

Demonstração. Suponha que (3.9) não ocorra, isto é,

$$
\left[w_{j}^{-}\left(t_{0}\right): 1 \leq j \leq n_{-}\right]+\left\{\psi \in H:\left\langle\psi, \eta_{k}^{+}\left(t_{0}\right)\right\rangle_{X}=0, \text { para } k=1, \cdots, m_{+}\right\} \neq H
$$

Considerando $\eta_{k}^{+}\left(t_{0}\right) \in X, k=1, \cdots, m_{+}$, como funcionais lineares sobre $H$, segue do resultado acima mencionado que existem constantes, não todas nulas, $c_{1}, \cdots, c_{m_{+}}$tais que

$$
\eta\left(t_{0}\right)=\sum_{k=1}^{m_{+}} c_{k} \eta_{k}^{+}\left(t_{0}\right) \quad \text { e }\left\langle\eta\left(t_{0}\right), w_{j}^{-}\left(t_{0}\right)\right\rangle_{X}=0, \text { para todo } j=1, \cdots, n_{-} .
$$

Considere $k_{0}, 1 \leq k_{0} \leq m_{+}$, de modo que $c_{k}=0$ para $k_{0}<k$. Segue do Corolário 1.5 que

$$
\begin{aligned}
\eta(t)= & \sum_{k=1}^{k_{0}} c_{k} \exp \left(-\int_{0}^{t} \lambda_{k}(s) d s\right)\left[\psi_{k}^{+}+o(1)\right] \\
= & \exp \left(-\int_{0}^{t} \lambda_{k_{0}}(s) d s\right)\left[\sum_{k=1}^{k_{0}-1} \exp \left(\int_{0}^{t}\left(\lambda_{k_{0}}(s)-\lambda_{k}(s)\right) d s\right)\left[c_{k} \psi_{k}^{+}+o(1)\right]+\right. \\
& \left.+c_{k_{0}} \psi_{k_{0}}^{+}+o(1)\right]
\end{aligned}
$$


quando $t \rightarrow+\infty$. Mas,

$$
\sum_{k=1}^{k_{0}-1} \exp \left(\int_{0}^{t}\left(\lambda_{k_{0}}(s)-\lambda_{k}(s)\right) d s\right)\left[c_{k} \psi_{k}^{+}+o(1)\right]=o(1)
$$

quando $t \rightarrow+\infty$, pois $0>\lambda_{k_{0}}(t)-\lambda_{k}(t) \rightarrow \lambda_{k_{0}}^{+}-\lambda_{k}^{+}<0$ quando $t \rightarrow+\infty$.

Assim,

$$
\eta(t)=\exp \left(-\int_{0}^{t} \lambda_{k_{0}}(s) d s\right)\left[c_{k_{0}} \psi_{k_{0}}^{+}+o(1)\right], \quad \text { quando } t \rightarrow+\infty,
$$

com convergência na norma de $C^{1}([0,1]) \times C^{1}([0,1])$.

Denotando $\eta(t)=(\theta(t), \vartheta(t))$ e a autofunção $\psi_{k_{0}}^{+}=\left(\psi_{1 k_{0}}^{+}, \psi_{2 k_{0}}^{+}\right)$, definimos as seguintes funções em $[-1,1]$

$$
\tilde{\eta}(x, t)=\left\{\begin{array}{l}
\theta(-x, t), x \in[-1,0] \\
\vartheta(x, t), x \in(0,1]
\end{array} \quad \text { e } \quad \tilde{\psi}_{k_{0}}^{+}(x)=\left\{\begin{array}{l}
\psi_{1 k_{0}}^{+}(-x), x \in[-1,0] \\
\psi_{2 k_{0}}^{+}(x), x \in(0,1]
\end{array} .\right.\right.
$$

Segue do Teorema 1.4 que $\tilde{\psi}_{k_{0}}^{+}$possui $k_{0}-1$ zeros simples em $(-1,1)$. Da convergência (3.11) na norma $C^{1}([0,1]) \times C^{1}([0,1])$ concluímos que $\tilde{\eta}(\cdot, t)$ possui $k_{0}-1$ zeros simples em $(-1,1)$ para $t$ suficientemente grande.

Por outro lado, aplicando o Teorema 1.8 a $\eta(t)$, com sinal de $t$ trocado, existem constantes $C \neq 0$ e inteiro $j \geq 1$ tais que

$$
\eta(t)=\exp \left(\int_{t}^{0} \lambda_{j}(s) d s\right)\left[C \psi_{j}^{-}+o(1)\right] \text { quando } t \rightarrow-\infty .
$$

De maneira análoga ao que fizemos quando $t \rightarrow+\infty$, obtemos de $(3.12)$ que $\tilde{\eta}(\cdot, t)$ possui $j-1$ zeros simples quando $t \rightarrow-\infty$.

Se $j \leq n_{-}$, então, da Observação 1.1 e de (3.10), obtemos

$$
\begin{aligned}
0 & =\left\langle\eta\left(t_{0}\right), w_{j}^{-}\left(t_{0}\right)\right\rangle_{X}=\left\langle\eta(t), w_{j}^{-}(t)\right\rangle_{X} \\
& =\left\langle\exp \left(\int_{t}^{0} \lambda_{j}(s) d s\right)\left[C \psi_{j}^{-}+o(1)\right], \exp \left(-\int_{t}^{0} \lambda_{j}(s) d s\right)\left[\psi_{j}^{-}+o(1)\right]\right\rangle_{X} \\
& =\left\langle C \psi_{j}^{-}, \psi_{j}^{-}\right\rangle_{X}+o(1),
\end{aligned}
$$

quando $t \rightarrow-\infty$. Como $\left\|\psi_{j}^{-}\right\|_{X}=1$, segue que $0=C+o(1)$, o que é um absurdo, já que $C \neq 0$. Portanto, $j>n_{-}$.

Finalmente, aplicando o Teorema 2.4 para a equação adjunta, com sinal de $t$ trocado, concluímos que $j-1 \leq k_{0}-1$, isto é, $j \leq k_{0}$, e assim $n_{-}<j \leq k_{0} \leq m_{+}$, o que contraria a hipótese. 
Reciprocamente, para que ocorra a transversalidade é necessário que

$\operatorname{dim}\left[w_{j}^{-}\left(t_{0}\right): 1 \leq j \leq n_{-}\right] \geq \operatorname{codim}\left\{\psi \in H:\left\langle\eta\left(t_{0}\right), w_{j}^{-}\left(t_{0}\right)\right\rangle_{X}=0, j=1, \cdots, n_{-}\right\}$, isto é, $n_{-} \geq m_{+}$.

Lema 3.3 Se $\bar{w}(t)$ e $\varphi_{ \pm}$são como no Lema 3.1 e $n_{-}$e $m_{+}$são os números de autovalores positivos da linearização em $\varphi_{-} e \varphi_{+}$, respectivamente, então $n_{-}>m_{+}$.

Demonstração. Como $\bar{w}(t)$ é uma solução não constante de (1.1) temos que $\bar{z}(t)=\bar{w}_{t}(t)$ é uma solução não nula de (3.6) sobre $-\infty<t<+\infty$ e, pelo Lema $3.1, \bar{z}(t) \rightarrow 0$ exponencialmente em $H$ quando $t \rightarrow \pm \infty$, isto é, $\bar{z}(t) \in T_{\bar{w}(t)} W^{u}\left(\varphi_{-}\right) \cap T_{\bar{w}(t)} W^{s}\left(\varphi_{+}\right)$ para todo $-\infty<t<+\infty$.

Aplicando o Teorema 1.8, $\frac{\bar{z}(t)}{\|\bar{z}(t)\|} \rightarrow \psi_{j}^{+}$quando $t \rightarrow+\infty$, para algum $j \geq 1$. Como $\bar{z}(t) \rightarrow 0$, segue que $\psi_{j}^{+}$está associada a um autovalor negativo, o que implica $j \geq m_{+}+1$.

Por outro lado, pelo Teorema 1.9 , existem constantes $C_{1}, \cdots, C_{n_{-}}$não todas nulas tais que $\bar{z}(t)=\sum_{j=1}^{n_{-}} C_{j} w_{j}^{-}(t)$. Seja $1 \leq n_{0} \leq n_{-}$tal que $C_{j}=0$ para todo $n_{0}<j$.

Assim,

$$
\begin{aligned}
\bar{z}(t)= & \sum_{j=1}^{n_{-}} C_{j} \exp \left(-\int_{t}^{0} \lambda_{j}(s) d s\right)\left[\psi_{j}^{-}+o(1)\right] \\
= & \exp \left(-\int_{t}^{0} \lambda_{n_{0}}(s) d s\right)\left[\sum_{j=1}^{n_{0}-1} \exp \left(-\int_{t}^{0}\left(\lambda_{j}(s)-\lambda_{n_{0}}(s)\right) d s\right)\left[C_{j} \psi_{j}^{-}+o(1)\right]+\right. \\
& \left.+C_{n_{0}} \psi_{n_{0}}^{-}+o(1)\right]
\end{aligned}
$$

quando $t \rightarrow-\infty$. Mas,

$$
\sum_{j=1}^{n_{0}-1} \exp \left(-\int_{t}^{0}\left(\lambda_{j}(s)-\lambda_{n_{0}}(s)\right) d s\right)\left[C_{j} \psi_{j}^{-}+o(1)\right]=o(1)
$$

quando $t \rightarrow-\infty$. Isto implica que $\bar{z}(t)=\exp \left(-\int_{t}^{0} \lambda_{n_{0}}(s) d s\right)\left[C_{n_{0}} \psi_{n_{0}}^{-}+o(1)\right]$ quando $t \rightarrow-\infty$, com convergência na norma de $C^{1}([0,1]) \times C^{1}([0,1])$.

Aplicando o Teorema 2.4 de forma análoga ao que fizemos na demonstração do Lema 3.2 , concluímos que $j-1 \leq n_{0}-1$, ou seja, $m_{+}<m_{+}+1 \leq j \leq n_{0} \leq n_{-}$, como queríamos demonstrar.

Finalmente enunciamos o resultado principal deste trabalho. 
Teorema 3.2 Sejam $a, b \in C^{1}([0,1],(0,+\infty))$ e $f, g$ satisfazendo as hipóteses $(H 1)$ $e\left(H_{2}\right)$. Se $\varphi_{-} e \varphi_{+}$são pontos de equilíbrio hiperbólicos do problema (1.1), então $W^{u}\left(\varphi_{-}\right) \pitchfork W^{s}\left(\varphi_{+}\right)$.

Demonstração. Suponha $\varphi_{-} \neq \varphi_{+}$. Se $W^{u}\left(\varphi_{-}\right) \cap W^{s}\left(\varphi_{+}\right)$é não vazio, existe uma solução $\bar{w}$ de $(1.1)$, sobre $-\infty<t<+\infty$, convergindo para $\varphi_{ \pm}$quando $t \rightarrow \pm \infty$. Como os equilíbrios são hiperbólicos, temos do Lema 3.3 que $n_{-}>m_{+}$. Portanto, segue do Lema 3.2 que $W^{u}\left(\varphi_{-}\right) \pitchfork_{\bar{w}(t)} W^{s}\left(\varphi_{+}\right)$, para cada $t$. Isto é válido para toda solução na interseção assim, $W^{u}\left(\varphi_{-}\right) \pitchfork W^{s}\left(\varphi_{+}\right)$quando a interseção é não vazia e trivialmente quando a interseção é vazia.

Se $\varphi_{-}=\varphi_{+}=\varphi$, segue do Lema 3.3 que não existe solução não constante convergindo para $\varphi$ quando $t \rightarrow \pm \infty$, isto é, não existe órbita homoclínica. Assim, $W^{u}(\varphi) \cap W^{s}(\varphi)=$ $\{\varphi\}$, que é trivialmente uma interseção transversal. 


\section{Capítulo 4}

\section{Bifurcação de pontos de equilíbrio}

\section{para um problema particular}

Neste capítulo, tentaremos descrever um pouco mais a dinâmica dos problemas da forma (0.1)-(0.3). Para isto, utilizaremos alguns resultados da teoria clássica de bifurcação, que serão enunciados no desenvolvimento do capítulo.

Suponha que as funções $a, b \in C^{1}([0,1],(0, \infty))$ em $(0.1)-(0.3)$ sejam constantes, digamos $a(x)=a$ e $b(x)=b$, para todo $x \in[0,1]$. Sem perda de generalidade, suponhamos que $0<a \leq b$. Então o problema (0.1)-(0.3) se reescreve como

$$
\left\{\begin{array}{l}
u_{t}=a u_{x x}+f(u), \quad x \in(0,1), t>0 \\
v_{t}=b v_{x x}+g(v), \quad x \in(0,1), t>0 \\
u(0, t)=v(0, t) \\
a u_{x}(0, t)+b v_{x}(0, t)=0 \\
u(1, t)=v(1, t)=0
\end{array}\right.
$$

É conveniente reescalarmos a variável $t$ fazendo $t \rightarrow a t$, assim (4.1) equivale ao problema

$$
\left\{\begin{array}{l}
u_{t}=u_{x x}+\lambda f(u), \quad x \in(0,1), t>0 \\
v_{t}=\alpha v_{x x}+\lambda g(v), \quad x \in(0,1), t>0 \\
u(0, t)=v(0, t) \\
u_{x}(0, t)+\alpha v_{x}(0, t)=0 \\
u(1, t)=v(1, t)=0
\end{array}\right.
$$

sendo $\alpha=b / a \geq 1$ e $\lambda=1 / a>0$. O objetivo agora, é considerarmos (4.2) como um 
problema de bifurcação sendo $\lambda>0$ o parâmetro de bifurcação e $\alpha \geq 1$ uma constante fixada. Mais precisamente, como $f(0)=g(0)=0,\left(\varphi_{0}, \psi_{0}\right)=(0,0)$ é um ponto de equilíbrio para qualquer valor de $\lambda>0$ e, portanto, estamos interessados em investigar os equilíbrios que bifurcam da curva de equilíbrios $(\lambda,(0,0))$.

Vamos nos restringir ao caso em que

$$
f(u)=u-c u^{3} \quad \text { e } g(v)=v-d v^{3}
$$

onde $c$ e $d$ são constantes positivas. Assim, um ponto de equilíbrio para o problema (4.2) é uma solução do seguinte sistema de segunda ordem

$$
\left\{\begin{array}{l}
\varphi^{\prime \prime}+\lambda\left(\varphi-c \varphi^{3}\right)=0, \quad x \in(0,1) \\
\alpha \psi^{\prime \prime}+\lambda\left(\psi-d \psi^{3}\right)=0, \quad x \in(0,1) \\
\varphi(0)=\psi(0) \\
\varphi^{\prime}(0)+\alpha \psi^{\prime}(0)=0 \\
\varphi(1)=\psi(1)=0,
\end{array}\right.
$$

Para cada $\lambda>0$, existe um atrator global $A_{\lambda}$. Primeiramente, mostraremos que o conjunto de equilíbrios $E_{\lambda}$ é limitado em $C([0,1]) \times C([0,1])$, uniformemente em $\lambda$. De fato, se $(\varphi, \psi)$ satisfaz o sistema (4.3) então multiplicando a primeira equação do sistema por $\varphi^{\prime}(x)$ e a segunda por $\psi^{\prime}(x)$ concluímos que $\varphi$ e $\psi$ devem satisfazer

$$
\begin{gathered}
\frac{\left(\varphi^{\prime}(x)\right)^{2}}{2}+\lambda F(\varphi(x))=\frac{1}{2}\left(\varphi^{\prime}(1)\right)^{2}, \quad x \in[0,1], \\
\frac{\alpha\left(\psi^{\prime}(x)\right)^{2}}{2}+\lambda G(\psi(x))=\frac{\alpha}{2}\left(\psi^{\prime}(1)\right)^{2}, x \in[0,1],
\end{gathered}
$$

onde $F(u)=\int_{0}^{u} f(s) d s=u^{2} / 2-c u^{4} / 4$ e $G(v)=\int_{0}^{v} g(s) d s=v^{2} / 2-d v^{4} / 4$. Em outras palavras, as funções $E_{1}(y, z)=z^{2} / 2+\lambda F(y)$ e $E_{2}(y, z)=\alpha z^{2} / 2+\lambda G(y)$ são integrais primeiras, respectivamente, da primeira e segunda equação do sistema (4.3).

Por outro lado, as constantes não - nulas $\varphi^{\prime}(1)$ e $\psi^{\prime}(1)$ devem ser tomadas de modo que $\varphi(0)=\psi(0), \varphi^{\prime}(0)=-\alpha \psi^{\prime}(0)$ e $\varphi(1)=\psi(1)=0$. Dessa forma, observando as curvas de nível das funções $E_{1}$ e $E_{2}$ obtemos como condição necessária que

$$
-\frac{1}{\min \{\sqrt{c}, \sqrt{d}\}} \leq \varphi(x), \psi(x) \leq \frac{1}{\min \{\sqrt{c}, \sqrt{d}\}}, \quad x \in[0,1] .
$$

Portanto, obtemos a limitação, uniforme em $\lambda$, do conjunto de pontos de equilíbrio $E_{\lambda}$ em $C([0,1]) \times C([0,1])$. 
Agora, consideremos o espaço de Banach

$Y=\left\{(\varphi, \psi) \in C^{2}([0,1]) \times C^{2}([0,1]): \varphi(0)=\psi(0), \varphi^{\prime}(0)+\alpha \psi^{\prime}(0)=0, \quad \varphi(1)=\psi(1)=0\right\}$

e a aplicação $J:(0, \infty) \times Y \rightarrow C([0,1]) \times C([0,1])$ definida por

$$
J(\lambda,(\varphi, \psi))=\left(\varphi^{\prime \prime}+\lambda\left(\varphi-c \varphi^{3}\right), \alpha \psi^{\prime \prime}+\lambda\left(\psi-d \psi^{3}\right)\right)=-A(\varphi, \psi)+\lambda h(\varphi, \psi)
$$

onde $A$ é o operador linear dado por $A(\varphi, \psi)=-\left(\varphi^{\prime \prime}, \alpha \psi^{\prime \prime}\right)$ e $h(\varphi, \psi)=\left(\varphi-c \varphi^{3}, \psi-\right.$ $\left.d \psi^{3}\right)$.

Nosso objetivo é estudar a estrutura do conjunto $J^{-1}(\{(0,0)\})$. Sabemos que $J(\lambda,(0,0))=(0,0)$, para todo $\lambda>0$, então, de acordo com o Teorema da Função Implícita, uma condição necessária para que um ponto $\left(\lambda_{0},(0,0)\right) \in(0, \infty) \times Y$ seja um ponto de bifurcação é que $D_{2} J\left(\lambda_{0},(0,0)\right)$ seja singular, isto é, não seja um homeomorfismo. Aqui, $D_{2} J\left(\lambda_{0},(0,0)\right)$ indica a derivada com relação ao segundo argumento da aplicação $J$. Porém, esta condição não é suficiente para que um ponto seja ponto de bifurcação; a seguir, enunciamos um resultado de Crandall e Rabinowitz (ver Teorema 1.7 de [6]), que nos dá uma condição suficiente para que um ponto $\left(\lambda_{0},(0,0)\right)$ seja um ponto de bifurcação.

Teorema 4.1 (Bifurcação de um autovalor simples ) Seja $U=S \times V$ um subconjunto aberto de $\mathbb{R} \times X$ e seja $F \in C^{2}(U, Y)$, onde $X$ e $Y$ são espaços de Banach. Suponha que $F(\lambda, 0)=0$, para todo $\lambda \in S$, e seja $L_{0}=D_{2} F\left(\lambda_{0}, 0\right)$ e $L_{1}=D_{1} D_{2} F\left(\lambda_{0}, 0\right)$. Assuma que as condições abaixo sejam satisfeitas:

(i) $\operatorname{dim} N\left(L_{0}\right)=\operatorname{codim} R\left(L_{0}\right)=1$;

(ii) $L_{1} u_{0} \notin R\left(L_{0}\right)$ onde $u_{0} \in X$ gera $N\left(L_{0}\right)$.

Seja $Z$ qualquer subespaço fechado de $X$ tal que $X=\operatorname{span}\left\{u_{0}\right\} \oplus Z$. Então existem uma constante $\delta>0$ e uma $C^{1}$-curva $(\lambda, \phi):(-\delta, \delta) \rightarrow \mathbb{R} \times Z$ tal que $\lambda(0)=\lambda_{0}, \phi(0)=0$ e, se $u(s)=s u_{0}+s \phi(s)$, então $F(\lambda(s), u(s))=0$. Além disso, existe uma vizinhança de $\left(\lambda_{0}, 0\right)$ tal que $F^{-1}(\{0\})$ consiste precisamente das curvas $(\lambda, 0)$ e $(\lambda(s), u(s)),|s|<\delta$.

As hipóteses (i) e (ii) do teorema acima implicam que $L_{0}=D_{2} F\left(\lambda_{0}, 0\right)$ tem 0 como um autovalor $L_{1}$-simples no sentido definido abaixo. 
Definição 4.1 Sejam $X$ e $Y$ espaços de Banach e $L_{0}, K \in \mathcal{L}(X, Y)$. Dizemos que $\mu \in \mathbb{C}$ é um autovalor $K$-simples de $L_{0}$ (com autofunção $u_{0}$ ) se

(a) $\operatorname{dim} N\left(L_{0}-\mu K\right)=\operatorname{codim} R\left(L_{0}-\mu K\right)=1$

e, se $N\left(L_{0}-\mu K\right)=\operatorname{span}\left\{u_{0}\right\}$

(b) $K u_{0} \notin R\left(L_{0}-\mu K\right)$.

Esta terminologia é motivada pelo caso em que $X=Y, K=I$ e $L_{0}$ é um operador compacto. Sob estas circunstâncias, $\mu \neq 0$ é um autovalor $I$-simples de $L_{0}$ se, e somente se, $\mu$ é um autovalor simples de $L_{0}$.

Veremos mais adiante, a importância desta noção, que indica que autovalores $K$ simples "continuam" ao longo dos ramos de equilíbrios bifurcados, isto é, para $s$ pequeno, $D_{2} F(\lambda(s), u(s))$ tem um único (pequeno) autovalor $\mu(s) K$-simples. Veremos também, um resultado que nos permite conhecer o sinal de $\mu(s)$, o que implica conhecer a estabilidade dos equilíbrios bifurcados.

Neste momento, verificamos as hipóteses do Teorema 4.1 para a aplicação $J$ definida em (4.7).

Sabemos que o operador linear $A$ definido por $A(\varphi, \psi)=-\left(\varphi^{\prime \prime}, \alpha \psi^{\prime \prime}\right)$ é um isomorfismo de $Y$ em $C([0,1]) \times C([0,1])$. Além disso, como $f$ e $g$ são suaves temos que $J$ também o é. Da definição temos que

$$
D_{2} J(\lambda,(0,0))(\varphi, \psi)=\left(\varphi^{\prime \prime}+\lambda \varphi, \alpha \psi^{\prime \prime}+\lambda \psi\right)=-A(\varphi, \psi)+\lambda(\varphi, \psi)
$$

e

$$
D_{1} D_{2} J(\lambda,(0,0))(\varphi, \psi)=(\varphi, \psi),
$$

$(\varphi, \psi) \in Y$, ou seja, $D_{2} J(\lambda,(0,0))=-A+\lambda I$ e $D_{1} D_{2} J(\lambda,(0,0))=I$. Então $D_{2} J(\lambda,(0,0))$ é singular se e somente se $\lambda=\lambda_{n}, n=1,2, \cdots$, onde $\left\{\lambda_{n}\right\}_{n}$ são os autovalores do operador $A$.

Vimos no Teorema 1.4, Capítulo 1 , que os autovalores de $A$ formam uma sequência crescente de números reais positivos $\lambda_{1}<\lambda_{2}<\cdots \rightarrow+\infty$, sendo todos autovalores simples. Fixado $n \in\{1,2, \cdots\}$, denotemos por $w_{n}=\left(u_{n}, v_{n}\right)$ a autofunção associada a $\lambda_{n}$. Não é difícil provar que

$$
N\left(D_{2} J\left(\lambda_{n},(0,0)\right)\right)=N\left(-A+\lambda_{n} I\right)=\operatorname{span}\left\{w_{n}\right\} \mathrm{e}
$$




$$
R\left(D_{2} J\left(\lambda_{n},(0,0)\right)\right)=\left\{(u, v) \in C([0,1]) \times C([0,1]): \int_{0}^{1}\left(u u_{n}+v v_{n}\right) d x=0\right\}
$$

Segue então que $D_{1} D_{2} J\left(\lambda_{n},(0,0)\right) w_{n}=w_{n}=\left(u_{n}, v_{n}\right) \notin R\left(D_{2} J\left(\lambda_{n},(0,0)\right)\right.$, para todo $n=1,2, \cdots$.

Agora, consideremos o subespaço $Z_{n}$ de $Y$ como sendo

$$
Z_{n}=\left\{(\varphi, \psi) \in Y: \int_{0}^{1}\left(\varphi u_{n}+\psi v_{n}\right) d x=0\right\}
$$

temos que $Y=\operatorname{span}\left\{w_{n}\right\} \oplus Z_{n}$ e todas as hipóteses do Teorema 4.1 estão satisfeitas para $\lambda_{0}=\lambda_{n}$. Portanto ocorre bifurcações em todos os pontos $\left(\lambda_{n},(0,0)\right), n \in\{1,2, \cdots\}$, isto é, para cada $n$ fixado existem uma constante $\delta_{n}>0$ e uma curva suave $\left(\lambda_{n}, \phi_{n}\right)$ : $\left(-\delta_{n}, \delta_{n}\right) \rightarrow \mathbb{R} \times Z_{n}$ tal que $\lambda_{n}(0)=\lambda_{n}, \phi_{n}(0)=0$ e, se $w_{n}(s)=s w_{n}+s \phi_{n}(s)=$ $s\left(u_{n}, v_{n}\right)+s\left(\phi_{1, n}(s), \phi_{2, n}(s)\right)$ então, $J\left(\lambda_{n}(s), w_{n}(s)\right)=0,|s|<\delta_{n}$. Além disso, existe uma vizinhança de $\left(\lambda_{n},(0,0)\right)$ tal que $J^{-1}(\{(0,0)\})$ consiste precisamente das curvas $(\lambda,(0,0))$ e $\left(\lambda_{n}(s), w_{n}(s)\right),|s|<\delta_{n}$. Calculemos a seguir os autovalores $\left\{\lambda_{n}\right\}_{n}$ do operador $A$, ou seja, para quais valores de $\lambda>0$ o sistema abaixo admite solução não - trivial.

$$
\left\{\begin{array}{l}
u^{\prime \prime}+\lambda u=0, \quad x \in(0,1) \\
\alpha v^{\prime \prime}+\lambda v=0, \quad x \in(0,1) \\
u(0)=v(0) \\
u^{\prime}(0)+\alpha v^{\prime}(0)=0 \\
u(1)=v(1)=0
\end{array}\right.
$$

Observe que o problema acima é a linearização do problema (4.3) em torno do equilíbrio trivial. Denotando $\lambda=\rho^{2}, \rho>0$ temos que as soluções das equações diferenciais em (4.8) são da forma

$$
\begin{gathered}
u(x)=C_{1} \cos (\rho x)+C_{2} \operatorname{sen}(\rho x), \quad x \in[0,1], \\
v(x)=C_{3} \cos (\rho x / \sqrt{\alpha})+C_{4} \operatorname{sen}(\rho x / \sqrt{\alpha}), \quad x \in[0,1],
\end{gathered}
$$

sendo $C_{i}, i=1, \cdots, 4$, constantes. Das condições de acoplamento em $x=0$, segue que $C_{1}=C_{3}$ e $C_{2}=-\sqrt{\alpha} C_{4}$, e fazendo $x=1$ obtemos

$$
\left\{\begin{array}{l}
C_{1} \cos \rho+C_{2} \operatorname{sen} \rho=0 \\
C_{1} \cos (\rho / \sqrt{\alpha})-C_{2} / \sqrt{\alpha} \operatorname{sen}(\rho / \sqrt{\alpha})=0 .
\end{array}\right.
$$


Para que o sistema acima tenha solução $\left(C_{1}, C_{2}\right) \neq(0,0)$ devemos ter

$$
\frac{1}{\sqrt{\alpha}} \cos \rho \operatorname{sen} \frac{\rho}{\sqrt{\alpha}}+\operatorname{sen} \rho \cos \frac{\rho}{\sqrt{\alpha}}=0 .
$$

Se $\cos \rho=0, \operatorname{sen} \rho= \pm 1$ e $\cos \rho / \sqrt{\alpha}=0$ e reciprocamente. Dessa forma, se $\cos \rho \neq 0$ a equação (4.9) pode ser reescrita como

$$
\tan \rho=-\frac{1}{\sqrt{\alpha}} \tan \frac{\rho}{\sqrt{\alpha}}
$$

Como $\alpha=b / a$ e $\lambda=1 / a=\rho^{2}$ temos que (4.10) é o mesmo que

$$
\frac{1}{\sqrt{a}} \tan \frac{1}{\sqrt{a}}=-\frac{1}{\sqrt{b}} \tan \frac{1}{\sqrt{b}} .
$$

A seguir temos no plano $a \times b$ as raízes da equação (4.11).

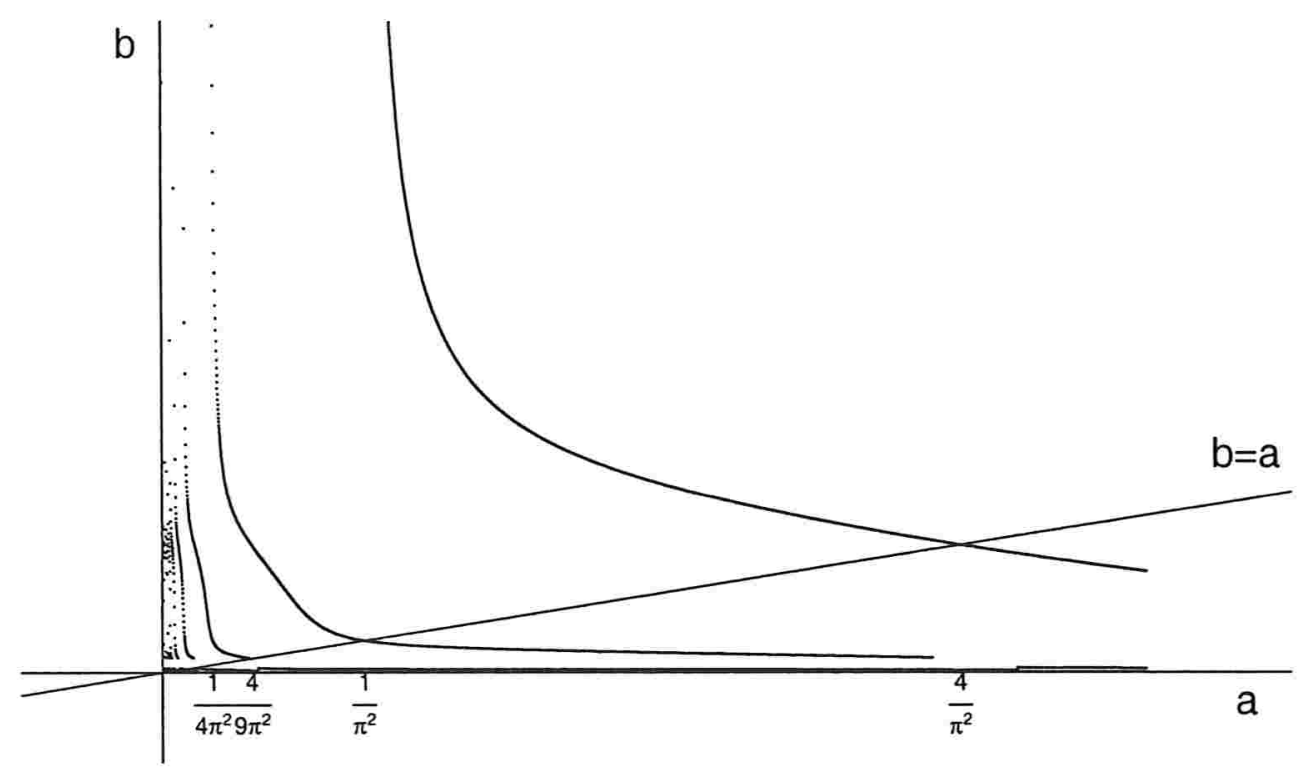

Algumas informações sobre a "direção" do ramo de soluções bifurcadas podem ser obtidas calculando $\left.\frac{d}{d s}\left(\lambda_{n}(s), w_{n}(s)\right)\right|_{s=0}=\left.\frac{d}{d s}\left(\lambda_{n}(s), s w_{n}+s \phi_{n}(s)\right)\right|_{s=0}=\left(\lambda_{n}^{\prime}(0), w_{n}\right)$. 
Calculemos o sinal de $\lambda_{n}^{\prime}(0)$. Para isto, substitua a curva suave $\left(\lambda_{n}(s), w_{n}(s)\right)=$ $=\left(\lambda_{n}(s), s\left(u_{n}, v_{n}\right)+s\left(\phi_{1, n}(s), \phi_{2, n}(s)\right)\right.$ no sistema (4.3). Assim, temos

$$
\begin{gathered}
\frac{d^{2}}{d x^{2}}\left(s u_{n}+s \phi_{1, n}(s)\right)+\lambda_{n}(s)\left[s u_{n}+s \phi_{1, n}(s)-c\left(s u_{n}+s \phi_{1, n}(s)\right)^{3}\right]=0 \\
\alpha \frac{d^{2}}{d x^{2}}\left(s v_{n}+s \phi_{2, n}(s)\right)+\lambda_{n}(s)\left[s v_{n}+s \phi_{2, n}(s)-d\left(s v_{n}+s \phi_{2, n}(s)\right)^{3}\right]=0 .
\end{gathered}
$$

Dividindo (4.12) e (4.13) por $s$, diferenciando com relação a $s$ e fazendo $s=0$ obtemos

$$
\begin{gathered}
\frac{d^{2}}{d x^{2}}\left[\phi_{1, n}^{\prime}(0)\right]+\lambda_{n}^{\prime}(0) u_{n}+\lambda_{n} \phi_{1, n}^{\prime}(0)=0 \\
\alpha \frac{d^{2}}{d x^{2}}\left[\phi_{2, n}^{\prime}(0)\right]+\lambda_{n}^{\prime}(0) v_{n}+\lambda_{n} \phi_{2, n}^{\prime}(0)=0
\end{gathered}
$$

onde o símbolo ' indica a derivada com relação a $s$. Multiplicando (4.14) e (4.15) por $u_{n}$ e $v_{n}$, respectivamente, integrando em $[0,1]$ e somando as expressões resultantes obtemos

$$
\begin{gathered}
\int_{0}^{1}\left\{\frac{d^{2}}{d x^{2}}\left[\phi_{1, n}^{\prime}(0)\right] u_{n}+\alpha \frac{d^{2}}{d x^{2}}\left[\phi_{2, n}^{\prime}(0)\right]\right\} d x+\lambda_{n}^{\prime}(0) \int_{0}^{1}\left(u_{n}^{2}+v_{n}^{2}\right) d x+ \\
+\lambda_{n} \int_{0}^{1}\left[\phi_{1, n}^{\prime}(0) u_{n}+\phi_{2, n}^{\prime}(0) v_{n}\right] d x=0
\end{gathered}
$$

Integrando por partes, duas vezes, o primeiro termo da equação acima e usando o fato que $w_{n}=\left(u_{n}, v_{n}\right)$ é uma autofunção do operador linear $A$ e que $\phi_{n}^{\prime}(0)=$ $\left(\phi_{1, n}^{\prime}(0), \phi_{2, n}^{\prime}(0)\right) \in Z_{n}$ concluímos que

$$
\lambda_{n}^{\prime}(0) \int_{0}^{1}\left(u_{n}^{2}+v_{n}^{2}\right) d x=0
$$

o que implica $\lambda_{n}^{\prime}(0)=0$, para $n \in\{1,2, \cdots\}$. Calculemos então, $\lambda_{n}^{\prime \prime}(0)$.

De forma análoga ao que fizemos para calcular $\lambda_{n}^{\prime}(0)$, porém diferenciando duas vezes com relação a $s$ as expressões (4.12) e (4.13), obtemos

$$
\begin{gathered}
\int_{0}^{1}\left\{\frac{d^{2}}{d x^{2}}\left[\phi_{1, n}^{\prime \prime}(0)\right] u_{n}+\alpha \frac{d^{2}}{d x^{2}}\left[\phi_{2, n}^{\prime \prime}(0)\right]\right\} d x+\lambda_{n}^{\prime \prime}(0) \int_{0}^{1}\left(u_{n}^{2}+v_{n}^{2}\right) d x+ \\
+\lambda_{n} \int_{0}^{1}\left[\phi_{1, n}^{\prime \prime}(0) u_{n}+\phi_{2, n}^{\prime \prime}(0) v_{n}\right] d x-2 \lambda_{n} \int_{0}^{1}\left[c u_{n}^{4}+d v_{n}^{4}\right] d x=0 .
\end{gathered}
$$

Integrando por partes, duas vezes, o primeiro termo da equação acima e usando o fato que $\phi_{n}^{\prime \prime}(0)=\left(\phi_{1, n}^{\prime \prime}(0), \phi_{2, n}^{\prime \prime}(0)\right) \in Z_{n}$ temos

$$
\lambda_{n}^{\prime \prime}(0) \int_{0}^{1}\left(u_{n}^{2}+v_{n}^{2}\right) d x=2 \lambda_{n} \int_{0}^{1}\left[c u_{n}^{4}+d v_{n}^{4}\right] d x .
$$


Como as constantes $c$ e $d$ são positivas, concluímos que $\lambda_{n}^{\prime \prime}(0)>0$, para $n \in\{1,2, \cdots\}$. Portanto, o gráfico para a função $\lambda_{n}(s)$ é da forma

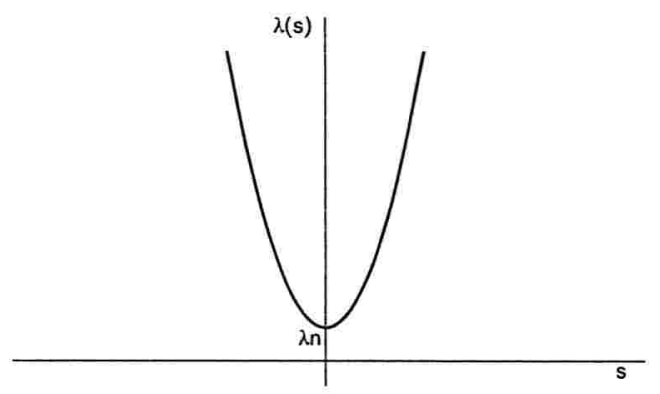

Assim, para cada $n \in\{1,2, \cdots\}$, existe uma vizinhaça de $\left(\lambda_{n},(0,0)\right)$ em $\mathbb{R} \times Y$ tal que o diagrama de bifurcação é da forma

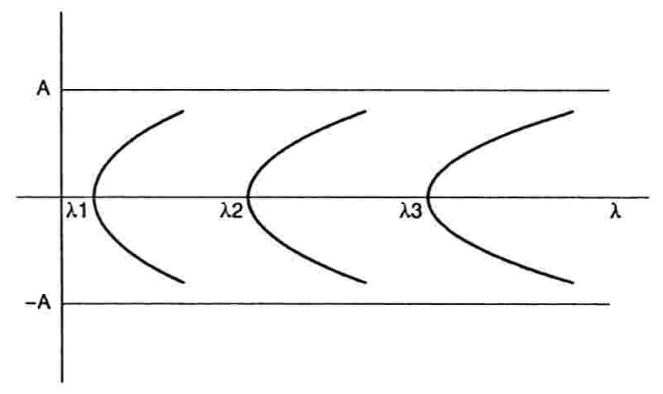

A seguir, provaremos um resultado sobre a hiperbolicidade dos equilíbrios não triviais. Como consequência desse resultado, poderemos concluir que não existe bifurcação secundária, isto é, nenhum outro ponto de equilíbrio pode bifurcar das curvas de equilíbrios que bifurcaram da origem.

Teorema 4.2 Seja $(x, \lambda) \longmapsto(\varphi(x, \lambda), \psi(x, \lambda))$ uma familia suave de equilibrios não triviais $((\varphi(., \lambda), \psi(., \lambda)) \neq(0,0)$ para cada $\lambda)$ do problema $(4.2)$, para $\lambda \in I \subset \mathbb{R}, I$ um intervalo. Então $\mu=0$ não é um autovalor da linearização em torno de $(\varphi(., \lambda), \psi(., \lambda))$. Em outras palavras, $(\varphi(., \lambda), \psi(., \lambda))$ é um equilibrio hiperbólico para todo $\lambda \in I$. 
Demonstração. Consideremos as seguintes equações linearizadas em torno de $(\varphi(., \lambda), \psi(., \lambda))$

$$
\begin{gathered}
u^{\prime \prime}+\lambda f^{\prime}(\varphi) u=0, \quad x \in(0,1) \\
\alpha v^{\prime \prime}+\lambda g^{\prime}(\psi) v=0, \quad x \in(0,1),
\end{gathered}
$$

acopladas em $x=0$ pelas condições

$$
\left\{\begin{array}{l}
u(0, \lambda)=v(0, \lambda) \\
u_{x}(0, \lambda)+\alpha v_{x}(0, \lambda)=0
\end{array}\right.
$$

e em $x=1$

$$
\{u(1, \lambda)=v(1, \lambda)=0 .
$$

Mostremos que o sistema (4.18)-(4.21) não admite solução não trivial. Para isto, reescrevemos o problema como um sistema de primeira ordem,

$$
\left\{\begin{array}{l}
u^{\prime}=w \\
w^{\prime}=-\lambda f^{\prime}(\varphi) u \\
v^{\prime}=z \\
z^{\prime}=-(\lambda / \alpha) g^{\prime}(\psi) v
\end{array}\right.
$$

cuja forma matricial é

$$
\left(\begin{array}{l}
u \\
w \\
v \\
z
\end{array}\right)^{\prime}=\left(\begin{array}{cccc}
0 & 1 & 0 & 0 \\
-\lambda f^{\prime}(\varphi) & 0 & 0 & 0 \\
0 & 0 & 0 & 1 \\
0 & 0 & -\frac{\lambda}{\alpha} g^{\prime}(\psi) & 0
\end{array}\right)\left(\begin{array}{l}
u \\
w \\
v \\
z
\end{array}\right)
$$

isto é, $U^{\prime}=A(x, \lambda) U$. Encontremos uma matriz fundamental para o problema acima. Obviamente as funções $\varphi_{x}(x, \lambda)$ e $\psi_{x}(x, \lambda)$ são soluções das equações (4.18) e (4.19), respectivamente. Com alguns cálculos simples verificamos que as funções definidas abaixo também são soluções das equações mencionadas acima. São elas

$$
\theta(x, \lambda)=2 \lambda \varphi_{\lambda}(x, \lambda)-x \varphi_{x}(x, \lambda) \text { e } \vartheta(x, \lambda)=2 \lambda \psi_{\lambda}(x, \lambda)-x \psi_{x}(x, \lambda) \text {, para } x \in(0,1)
$$

Assim, definimos a matriz 


$$
U(x, \lambda)=\left(\begin{array}{cccc}
\varphi_{x} & 2 \lambda \varphi_{\lambda}-x \varphi_{x} & 0 & 0 \\
\varphi_{x x} & 2 \lambda \varphi_{\lambda x}-\varphi_{x}-x \varphi_{x x} & 0 & 0 \\
0 & 0 & \psi_{x} & 2 \lambda \psi_{\lambda}-x \psi_{x} \\
0 & 0 & \psi_{x x} & 2 \lambda \psi_{\lambda x}-\psi_{x}-x \psi_{x x}
\end{array}\right)
$$

Se $U(x, \lambda)$ é uma matriz fundamental então, qualquer solução do problema (4.23) é dada por

$$
\left(\begin{array}{c}
u(x, \lambda) \\
u_{x}(x, \lambda) \\
v(x, \lambda) \\
v_{x}(x, \lambda)
\end{array}\right)=\left(\begin{array}{cccc}
\varphi_{x} & 2 \lambda \varphi_{\lambda}-x \varphi_{x} & 0 & 0 \\
\varphi_{x x} & 2 \lambda \varphi_{\lambda x}-\varphi_{x}-x \varphi_{x x} & 0 & 0 \\
0 & 0 & \psi_{x} & 2 \lambda \psi_{\lambda}-x \psi_{x} \\
0 & 0 & \psi_{x x} & 2 \lambda \psi_{\lambda x}-\psi_{x}-x \psi_{x x}
\end{array}\right)\left(\begin{array}{c}
u(0, \lambda) \\
u_{x}(0, \lambda) \\
v(0, \lambda) \\
v_{x}(0, \lambda)
\end{array}\right)
$$

e usando as condições em (4.20) temos

$$
\left(\begin{array}{c}
u(x, \lambda) \\
u_{x}(x, \lambda) \\
v(x, \lambda) \\
v_{x}(x, \lambda)
\end{array}\right)=\left(\begin{array}{cccc}
\varphi_{x} & 2 \lambda \varphi_{\lambda}-x \varphi_{x} & 0 & 0 \\
\varphi_{x x} & 2 \lambda \varphi_{\lambda x}-\varphi_{x}-x \varphi_{x x} & 0 & 0 \\
0 & 0 & \psi_{x} & 2 \lambda \psi_{\lambda}-x \psi_{x} \\
0 & 0 & \psi_{x x} & 2 \lambda \psi_{\lambda x}-\psi_{x}-x \psi_{x x}
\end{array}\right)\left(\begin{array}{c}
v(0, \lambda) \\
-\alpha v_{x}(0, \lambda) \\
v(0, \lambda) \\
v_{x}(0, \lambda)
\end{array}\right) .
$$

Ou seja, para $x \in[0,1]$

$$
u(x, \lambda)=\varphi_{x}(x, \lambda) v(0, \lambda)-2 \alpha \lambda \varphi_{\lambda}(x, \lambda) v_{x}(0, \lambda)+\alpha x \varphi_{x}(x, \lambda) v_{x}(0, \lambda)
$$

e

$$
v(x, \lambda)=\psi_{x}(x, \lambda) v(0, \lambda)+2 \lambda \psi_{\lambda}(x, \lambda) v_{x}(0, \lambda)-x \psi_{x}(x, \lambda) v_{x}(0, \lambda)
$$

Fazendo $x=1$ nas equações acima, segue da condição (4.21) que

$$
\left\{\begin{array}{c}
\varphi_{x}(1, \lambda) v(0, \lambda)+\alpha \varphi_{x}(1, \lambda) v_{x}(0, \lambda)=0 \\
\psi_{x}(1, \lambda) v(0, \lambda)-\psi_{x}(1, \lambda) v_{x}(0, \lambda)=0
\end{array}\right.
$$

pois $\varphi_{\lambda}(1, \lambda)=\psi_{\lambda}(1, \lambda)=0$. Como $(\varphi(., \lambda), \psi(., \lambda)) \neq 0$, temos $\varphi_{x}(1, \lambda) \neq 0$ e $\psi_{x}(1, \lambda) \neq$ 0 , o que implica

$$
\left\{\begin{array}{l}
v(0, \lambda)+\alpha v_{x}(0, \lambda)=0 \\
v(0, \lambda)-v_{x}(0, \lambda)=0
\end{array}\right.
$$


Resolvendo o sistema obtemos que $v(0, \lambda)=v_{x}(0, \lambda)=0$; novamente das condições (4.20) obtemos $u(0, \lambda)=u_{x}(0, \lambda)=0$. Portanto, segue que $(u(., \lambda), v(., \lambda))=(0,0)$, para todo $\lambda \in I$.

Faltou provar que, realmente, a matriz $U(x, \lambda)$ é uma matriz fundamental, isto é, que suas colunas são linearmente independentes. Provemos isto para as primeira e segunda colunas, o restante é análogo.

Suponha por absurdo, que existe uma constante $C$ tal que

$$
\varphi_{x}(x, \lambda)=2 C \lambda \varphi_{\lambda}(x, \lambda)-C x \varphi_{x}(x, \lambda), \quad x \in[0,1] .
$$

Fazendo $x=1$ obtemos

$$
\varphi_{x}(1, \lambda)=2 C \lambda \varphi_{\lambda}(1, \lambda)-C \varphi_{x}(1, \lambda)=-C \varphi_{x}(1, \lambda), \quad x \in[0,1]
$$

ou seja, $(1+C) \varphi_{x}(1, \lambda)=0$ o que implica, $C=-1$. Assim,

$$
\varphi_{x}(x, \lambda)=-2 \lambda \varphi_{\lambda}(x, \lambda)+x \varphi_{x}(x, \lambda), \quad x \in[0,1]
$$

isto é,

$$
(x-1) \varphi_{x}(x, \lambda)-2 \lambda \varphi_{\lambda}(x, \lambda)=0, \quad x \in[0,1] .
$$

Utilizando o método das curvas características resolvemos a equação diferencial (4.24).

$$
\begin{cases}x^{\prime}(s)=x-1, & x(0)=0 \\ \lambda^{\prime}(s)=-2 \lambda, & \lambda(0)=\xi \\ \varphi^{\prime}(s)=0, & \varphi(0)=\varphi(0, \xi)=h(\xi) .\end{cases}
$$

Resolvendo o sistema acima obtemos

$$
\left\{\begin{array}{l}
x(s)=1-e^{s} \\
\lambda(s)=\xi e^{-2 \lambda} \\
\varphi(s)=\varphi(x(s), \lambda(s))=\varphi(0, \xi)=h(\xi) .
\end{array}\right.
$$

Agora, explicitemos $\xi$

$$
\left\{\begin{array}{l}
x=1-e^{s} \Rightarrow s=\ln (1-x) \\
\lambda=\xi e^{-2 \lambda} \Rightarrow \xi=\lambda e^{2 \lambda}=\lambda(1-x)^{2}
\end{array}\right.
$$

logo, $\varphi(x, \lambda)=h(\xi)=h\left(\lambda(1-x)^{2}\right)$. Diferenciando com relação a $x$,

$$
\varphi_{x}(x, \lambda)=h^{\prime}\left(\lambda(1-x)^{2}\right)(-2 \lambda(1-x))
$$


e fazendo $x=1$ concluímos que $\varphi_{x}(1, \lambda)=0$, contradizendo o fato de que $\varphi(., \lambda) \neq 0$. Portanto, as primeiras e segundas colunas são linearmente independentes.

Logo após a Definição 4.1 fizemos alguns comentários sobre a importância da noção de autovalor $K$-simples para o estudo da estabilidade dos pontos de equilíbrios bifurcados. Neste momento pretendemos esclarecer estes comentários. Para isto, enunciamos alguns resultados de [7] sem incluir as demonstrações .

Lema 4.1 Sejam $X, Y$ espaços de Banach e $L_{0}, K \in \mathcal{L}(X, Y)$. Considere $\mu_{0}$ um autovalor $K$-simples de $L_{0}$, com autofunção $w_{0}$. Então existe $\rho>0$ tal que se $\left\|L-L_{0}\right\|<$ $\rho, L$ tem um único autovalor $K$-simples $\eta(L)$, com autofunção $w(L)=w_{0}+z(L)$; aqui $X=\operatorname{span}\left\{w_{0}\right\} \oplus Z$, e $z(L) \in Z$. Também, $\eta\left(L_{0}\right)=\mu_{0}, w\left(L_{0}\right)=w_{0}$ e a aplicação $L \longmapsto(w(L), \eta(L))$ é suave.

A seguir temos um corolário deste lema. Os dados $F, Z$, $u_{0}$ são como no Teorema 4.1, e $\lambda(s), u(s)$ são as funções do referido teorema. Escrevemos $\eta(s)=\eta\left(D_{2} F(\lambda(s), u(s))\right)$ e $w(s)=w\left(D_{2} F(\lambda(s), u(s))\right)$.

Corolário 4.1 Suponha que $X \subset Y$ com inclusão $I: X \rightarrow Y$ contínua. Se $D_{2} F\left(\lambda_{0}, 0\right)$ tem 0 como um autovalor I-simples então existem funções suaves

$$
\lambda \longmapsto(\gamma(\lambda), v(\lambda)) \quad \text { e } s \longmapsto(\eta(s), w(s))
$$

definidas em vizinhanças de $\lambda_{0}$ e 0 , respectivamente, sobre $\mathbb{R} \times X$ tais que

$$
\begin{gathered}
D_{2} F(\lambda, 0) v(\lambda)=\gamma(\lambda) v(\lambda), \\
D_{2} F(\lambda(s), u(s)) w(s)=\eta(s) w(s) .
\end{gathered}
$$

Além disso, $\gamma\left(\lambda_{0}\right)=\eta(0)=0, v\left(\lambda_{0}\right)=u_{0}=w(0)$ e $v(\lambda)-u_{0} \in Z, w(s)-u_{0} \in Z$.

Tomando $X=\left\{(\varphi, \psi) \in C^{2}([0,1])^{2}: \varphi(0)=\psi(0), \varphi^{\prime}(0)=-\alpha \psi^{\prime}(0), \varphi(1)=\psi(1)=\right.$ $0\}, Y=C([0,1])^{2}, F=J$ e $\lambda_{0}=\lambda_{n}, n=1,2, \cdots$, autovalores do operador $A$, estamos nas condições do corolário acima. Observe que a equação (4.25) é a linearização de (4.3) em torno de $(\lambda, 0)$ e estudamos o espectro quando $\lambda$ cruza $\lambda_{0}$, enquanto que (4.26) é a linearização em torno dos equilíbrios bifurcados e o espectro é estudado para $s$ próximo 
de $s=0$. Dessa forma, o sinal de $\eta(s)$ determina a estabilidade dos equilíbrios bifurcados. O teorema a seguir nos dá uma maneira prática para determinarmos tal sinal.

Teorema 4.3 Com as mesmas hipóteses do Teorema 4.1, considere $\gamma$ e $\eta$ as funções provenientes do Corolário 4.1. Então $\gamma^{\prime}\left(\lambda_{0}\right) \neq 0$ e se $\eta(s) \neq 0$, para s próximo de 0 ,

$$
\lim _{s \rightarrow 0} \frac{s \lambda^{\prime}(s) \gamma^{\prime}\left(\lambda_{0}\right)}{\eta(s)}=-1 \text {. }
$$

Em outras palavras, sgn $\eta(s)=-\operatorname{sgn} s \lambda^{\prime}(s) \gamma^{\prime}\left(\lambda_{0}\right)$.

Demonstramos no Teorema 4.2 que os pontos de equilíbrio não - triviais, que constituem famílias suaves, são todos hiperbólicos, ou seja, $\eta(s) \neq 0$ para todo $s$ no intervalo de definição de $(\lambda(s), w(s))$. Assim, estamos nas condições do teorema acima, o qual pretendemos aplicar para $\lambda_{0}=\lambda_{n}, n=1,2, \cdots$.

Como a função $\gamma(\lambda)$ é definida numa vizinhança de $\lambda_{0}=\lambda_{n}$, faremos a seguinte mudança de variável

$$
\tilde{\lambda}=\lambda-\lambda_{n}
$$

Assim, $\tilde{\lambda}^{\prime}(s)=\lambda^{\prime}(s)$ e $J(\tilde{\lambda},(\varphi, \psi))=-A(\varphi, \psi)+\left(\tilde{\lambda}+\lambda_{n}\right)(\varphi, \psi)$, o que implica

$$
D_{2} J(\tilde{\lambda},(0,0))(u, v)=-A(u, v)+\left(\tilde{\lambda}+\lambda_{n}\right)(u, v) .
$$

Em particular, fazendo $(u, v)=\left(u_{n}, v_{n}\right)$, autofunção associada a $\lambda_{n}$, obtemos

$$
D_{2} J(\tilde{\lambda},(0,0))\left(u_{n}, v_{n}\right)=-A\left(u_{n}, v_{n}\right)+\left(\tilde{\lambda}+\lambda_{n}\right)\left(u_{n}, v_{n}\right)=\tilde{\lambda}\left(u_{n}, v_{n}\right) .
$$

Portanto, da equação (4.25), segue que $\gamma(\tilde{\lambda})=\tilde{\lambda}$, o que implica, $\gamma^{\prime}\left(\lambda_{0}\right)=1$.

Por outro lado, vimos anteriormente que $\lambda^{\prime}(0)=0$ e que $\lambda^{\prime \prime}(0)>0$. Isto implica que sgn $s \lambda^{\prime}(s)=1$, para $s$ próximo de 0 . Mas $\tilde{\lambda}^{\prime}(s)=\lambda^{\prime}(s)$, ou seja, sgn $s \tilde{\lambda}(s)=\operatorname{sgn}$ $s \lambda(s)=1$. Portanto, segue do Teorema 4.3, que sgn $\eta(s)=-1$, para $s$ próximo de 0 ; isto implica que o primeiro par de equilíbrios bifurcados $\left(\varphi_{1}^{ \pm}, \psi_{1}^{ \pm}\right)$são assintoticamente estáveis e os outros pares de equilíbrios $\left(\varphi_{j}^{ \pm}, \psi_{j}^{ \pm}\right), j=2, \cdots$, são instáveis. Localmente podemos afirmar que $\operatorname{dim} W^{u}\left(\varphi_{j}^{ \pm}, \psi_{j}^{ \pm}\right)=j-1, j=1, \cdots$.

Observemos que se $0<\lambda \leq \lambda_{1}$ e $(\varphi, \psi)$ é um ponto de equilíbrio de (1.1) então

$$
\begin{aligned}
\lambda \int_{0}^{1}\left(c \varphi^{4}+d \psi^{4}\right) d x & =\int_{0}^{1}\left(\varphi^{\prime \prime} \varphi+\alpha \psi^{\prime \prime} \psi+\lambda(\varphi+\psi)\right) d x \\
& =\int_{0}^{1}\left(-\left(\varphi^{\prime}\right)^{2}-\alpha\left(\psi^{\prime}\right)^{2}+\lambda\left(\varphi^{2}+\psi^{2}\right)\right) d x \leq 0
\end{aligned}
$$


isto é, $(\varphi, \psi)=(0,0)$. Portanto, o atrator global para $0<\lambda \leq \lambda_{1}$ é dado por $\mathcal{A}=$ $\{(0,0)\}$.

A seguir, veremos que os ramos de pontos de equilíbrio são ilimitados em $(0, \infty) \times$ $(C([0,1]) \times C([0,1]))$. Como no início deste capítulo, provamos que o conjunto de pontos de equilíbrio $E_{\lambda}$ é limitado, uniformemente em $\lambda$, na norma de $C([0,1]) \times C([0,1])$, teremos que a ilimitação acima, se dá no argumento $\lambda$. Em outras palavras, os ramos de pontos de equilíbrio, que bifurcam do equilíbrio trivial, "continuam" infinitamente.

Sabemos que o operador linear

$$
A: Y=\left\{(\varphi, \psi) \in C^{2}([0,1]) \times C^{2}([0,1]):+(C F)\right\} \longrightarrow C([0,1]) \times C([0,1])
$$

é um isomorfismo e que $A^{-1}: C([0,1]) \times C([0,1]) \rightarrow Y \subset C([0,1]) \times C([0,1])$ é um operador linear compacto em $C([0,1]) \times C([0,1])$. Assim, aplicando $-A^{-1}$ na aplicação $J$ obtemos

$$
\tilde{J}(\lambda,(\varphi, \psi))=-A^{-1} J(\lambda,(\varphi, \psi))=(\varphi, \psi)-\lambda A^{-1} h(\varphi, \psi) .
$$

Por outro lado, $h(\varphi, \psi)=h(0,0)+h^{\prime}(0,0)(\varphi, \psi)+\tilde{h}(\varphi, \psi)=(\varphi, \psi)+\tilde{h}(\varphi, \psi)$, onde $\tilde{h}(\varphi, \psi)=o\left(\|(\varphi, \psi)\|_{\infty}\right)$ quando $\|(\varphi, \psi)\|_{\infty} \rightarrow 0$. Logo,

$$
\tilde{J}(\lambda,(\varphi, \psi))=(\varphi, \psi)-\lambda A^{-1}(\varphi, \psi)-\lambda A^{-1} \tilde{h}(\varphi, \psi)
$$

Enunciamos, a seguir, mais um resultado de Rabinowitz (Teorema 1.3 de [17]) que versa sobre aspectos globais das soluções bifurcadas.

Teorema 4.4 Sejam B um espaço de Banach e $F \in C(U, B)$ onde $U$ é um suconjunto aberto de $\mathbb{R} \times B$. Assuma que $F$ é da seguinte forma

$$
F(\lambda, w)=w-\lambda L w+h(\lambda, w)
$$

onde

(i) $1 / \lambda_{0}$ é um autovalor de $L$ com multiplicidade impar;

(ii) $L: B \rightarrow B$ é um operador linear compacto;

(iii) $h: U \rightarrow B$ é compacta;

(iv) $h(\lambda, w)=o(\|w\|)$ em $w=0$, uniformemente para $\lambda$ em intervalos limitados. 
Denote por $S$ o fecho do conjunto de zeros não - triviais de F. Então $S$ contém uma componente $C$ (isto é, um subconjunto conexo maximal) que encontra $\left(\lambda_{0}, 0\right)$ e ou

(I) $C$ é não - compacto em $U$ (se $U=\mathbb{R} \times B$ isto indica que $C$ é ilimitado),

ou

(II) $C$ encontra $w=0$ em um ponto $(\bar{\lambda}, 0)$ onde $\bar{\lambda} \neq \lambda_{0}$ e $1 / \bar{\lambda}$ é um autovalor de $L$.

Para o nosso caso, $B=C([0,1]) \times C([0,1]), L=A^{-1}, h(\lambda, w)=-\lambda \tilde{h}(w)$ e $\lambda_{0}=\lambda_{n}$, $n=1,2, \cdots$. Gostaríamos de verificar que a condição (I), do teorema acima, é válida para o nosso caso. Para isso, vamos provar que (II) não ocorre. Lembremos que os pontos de equilíbrio bifurcados em $\lambda_{0}=\lambda_{n}, n=1,2, \cdots$, são dados por $\left(\lambda(s), w_{n}(s)\right)$, para $s$ numa vizinhança de 0 , onde

$$
w_{n}(s)=s\left(u_{n}, v_{n}\right)+s\left(\phi_{1, n}(s), \phi_{2, n}(s)\right)
$$

sendo $\phi_{n}(s)=\left(\phi_{1, n}(s), \phi_{2, n}(s)\right) \in Z_{n}=\left\{(\varphi, \psi) \in Y: \int_{0}^{1}\left(\varphi u_{n}+\psi v_{n}\right) d x=0\right\}$ e $\left(u_{n}, v_{n}\right)$ autofunção de $A$ associada ao autovalor $\lambda_{n}$.

Para cada $s$, definimos a função $\tilde{w}_{n}(s):[-1,1] \rightarrow \mathbb{R}$ por

$$
\left[\tilde{w}_{n}(s)\right](x)=\left\{\begin{array}{l}
s u_{n}(-x)+s\left[\phi_{1, n}(s)\right](-x), \quad x \in[-1,0] \\
s v_{n}(x)+s\left[\phi_{2, n}(s)\right](x), \quad x \in[0,1]
\end{array}\right.
$$

isto é, $\tilde{w}_{n}(s)=s \tilde{w}_{n}+s \tilde{\phi}_{n}(s)$, onde $\tilde{w}_{n}(x)=\left\{\begin{array}{l}u_{n}(-x), x \in[-1,0] \\ v_{n}(x), x \in[0,1]\end{array}\right.$ é a autofunção, associada ao autovalor $\lambda_{n}$, do operador $\tilde{A}: D(\tilde{A}) \subset L^{2}(-1,1) \rightarrow L^{2}(-1,1)$ definido por

$$
\tilde{A} w(x)=\left\{\begin{array}{l}
w^{\prime \prime}(x), \quad x \in(-1,0) \\
\alpha w^{\prime \prime}(x), \quad x \in(0,1)
\end{array}\right.
$$

considerado na Seção 1.2; em tal seção, provamos que $\tilde{w}_{n}$ possui exatamente $n-1$ zeros simples em $(-1,1)$.

Como $\tilde{w}_{n}(s)=s \tilde{w}_{n}+s \tilde{\phi}_{n}(s)$ temos, para $|s|$ pequeno, que $\tilde{w}_{n}(s)$ possui exatamente 
$n-1$ zeros. Lembremos que, para cada $s, \tilde{w}_{n}(s)$ é uma solução do problema

$$
\left\{\begin{array}{l}
w^{\prime \prime}+\lambda_{n}(s)\left(w-c w^{3}\right)=0, \quad x \in(-1,0) \\
\alpha w^{\prime \prime}+\lambda_{n}(s)\left(w-d w^{3}\right)=0, \quad x \in(0,1) \\
w(0-)=w(0+) \\
w^{\prime}(0-)=\alpha w^{\prime}(0+) \\
w(-1)=w(1)=0
\end{array}\right.
$$

e, portanto, como $\tilde{w}_{n}(s)$ é não identicamente nula, não pode existir $\bar{x} \in[-1,1]$ tal que

$$
\left[\tilde{w}_{n}(s)\right](\bar{x})=\frac{d}{d x}\left[\tilde{w}_{n}(s)\right](\bar{x})=0
$$

pois isto contrariaria o teorema de unicidade de solução. Dessa forma, o ramo de pontos de equilíbrio bifurcados contendo $\left(\lambda_{n},(0,0)\right)$ não pode encontrar $\left(\lambda_{k},(0,0)\right)$, para todo $k \neq n, k=1,2, \cdots$; em outras palavras, a condição (II) do Teorema 4.4 não ocorre, o que implica que a condição (I) é válida, isto é, o ramo de equilíbrios $\left(\lambda(s), w_{n}(s)\right)$ é ilimitado em $(0, \infty) \times(C([0,1]) \times C([0,1]))$.

Resumimos os resultados deste capítulo, no seguinte

Teorema 4.5 Sejam $\alpha \geq 1, \lambda_{0}=0, f(u)=u-c u^{3}$ e $g(v)=v-d v^{3}, u, v \in \mathbb{R}$, onde c e d são constantes positivas. Se $\lambda \in\left(\lambda_{n}, \lambda_{n+1}\right), n=1,2, \cdots$, existem pelo menos $2 n+1$ pontos de equilibrio, $\left(\varphi_{0}, \psi_{0}\right)=(0,0),\left(\varphi_{j}^{+}, \psi_{j}^{+}\right) e\left(\varphi_{j}^{-}, \psi_{j}^{-}\right), j=1,2, \cdots, n$, do problema (4.2) e, para $\lambda$ próximo de $\lambda_{n}$, os pontos $\left(\varphi_{n}^{ \pm}, \psi_{n}^{ \pm}\right)$são hiperbólicos com $\operatorname{dim} W^{u}\left(\left(\varphi_{n}^{ \pm}, \psi_{n}^{ \pm}\right)\right)=n-1$.

Se $\lambda \in\left(\lambda_{n}, \lambda_{n+1}\right)$, então $\left(\varphi_{0}, \psi_{0}\right)=(0,0)$ é ponto de equilíbrio hiperbólico, $\operatorname{dim} W^{u}(0,0)=n, n=0,1,2, \cdots ;$ para $0<\lambda \leq \lambda_{1}$, o atrator $A_{\lambda}=\{(0,0)\}$.

Rocha em [18] estuda um problema de bifurcação bastante similar ao problema (4.2), com condição de fronteira de Neumann no ponto $x=1$ e $f(s)=g(s)=s-s^{3}, s \in \mathbb{R}$. De forma analítica, ele consegue descrever o diagrama de bifurcação sem utilizar os resultados clássicos de Rabinowitz e consegue também dar mais informações sobre o número de equilíbrios. 


\section{Capítulo 5}

\section{Conclusões e Observações}

Nosso objetivo, neste capítulo, é discutirmos alguns problemas similares ao que foi estudado neste trabalho.

Seja a uma função positiva definida no intervalo $[0,1]$ tal que existem

$$
x_{0}=0<x_{1}<\cdots<x_{j-1}<x_{j}<\cdots<x_{n}=1
$$

de forma que $\left.a\right|_{\left(x_{j-1}, x_{j}\right)}$ pode ser prolongada a uma função de classe $C^{1}$ em $\left[x_{j-1}, x_{j}\right]$, para todo $j=1, \cdots, n$. Além disso, suponha que existe uma constante $M$ tal que $0<1 / M \leq a(x) \leq M$, para todo $x \in[0,1]$.

Podemos agora, considerar o seguinte problema parabólico

$$
\left\{\begin{array}{l}
u_{t}=\left(a(x) u_{x}\right)_{x}+f(u), x \in(0,1), x \neq x_{j}, j=1, \cdots, n-1, t>0 \\
u(0, t)=u(1, t)=0
\end{array}\right.
$$

onde $f$ satisfaz as hipóteses $(H 1)$ e $(H 2)$. Por causa da possível descontinuidade da função $a$, complementamos o problema (5.1) com a seguinte condição : se $v=a(x) u_{x}$, então $u$ e $v$ são contínuas em $[0,1]$, isto é, $u\left(x_{j}^{-}, t\right)=u\left(x_{j}^{+}, t\right)$ e $a\left(x_{j}^{-}\right) u_{x}\left(x_{j}^{-}, t\right)=$ $a\left(x_{j}^{+}\right) u_{x}\left(x_{j}^{+}, t\right)$.

Não é difícil provar que o problema acima define um $C^{1,1}$-semigrupo gradiente $T(t)$ em $H_{0}^{1}(0,1)$ e que existe um atrator global conexo $\mathcal{A}=\mathcal{A}_{a, f} \subset H_{0}^{1}(0,1)$.

Com adaptações devidas, a transversalidade das variedades estável e instável dos pontos de equilíbrio hiperbólicos para o problema (5.1) pode ser obtida com os mesmos argumentos utilizados para o problema (0.1)-(0.3). 
Outro problema semelhante ao estudado nesse trabalho é o seguinte

$$
\left\{\begin{array}{l}
u_{t}=\frac{1}{a(x)}\left(a(x) u_{x}\right)_{x}+f(u), \quad x \in(0,1), t>0 \\
v_{t}=\frac{1}{b(x)}\left(b(x) v_{x}\right)_{x}+g(v), \quad x \in(0,1), t>0 \\
u(0, t)=u(0, t) \\
a(0) u_{x}(0, t)+b(0) v_{x}(0, t)=0 \\
u(1, t)=v(1, t)=0
\end{array}\right.
$$

onde $a, b \in C^{1}([0,1],(0,+\infty))$ e $f, g$ satisfazem $(H 1)$ e $(H 2)$. Um problema similar a (5.2)foi considerado em [10] como um problema limite de uma equação parabólica considerada num domínio "fino" bidimensional em formato de "L". Se considerarmos $X=L^{2}(0,1) \times L^{2}(0,1)$ e as funções $a$ e $b$ como pesos na norma de $X$, isto é,

$$
\|(u, v)\|_{X}^{2}=\int_{0}^{1} a(x)|u(x)|^{2} d x+\int_{0}^{1} b(x)|v(x)|^{2} d x
$$

podemos tratar o sistema (5.2) da mesma forma com que tratamos (0.1)-(0.3) e obtermos a transversalidade das variedades estável e instável de pontos de equilíbrio hiperbólicos. O fato do sistema (5.2) ser equivalente a uma equação unidimensional é crucial para podermos utilizar as mesmas técnicas.

Por outro lado, existem classes de sistemas que, de certa forma, generalizam o problema (0.1)-(0.3), para os quais não conseguimos aplicar as mesmas idéias. Um exemplo é o sistema

$$
\left\{\begin{array}{l}
u_{t}=\left(a(x) u_{x}\right)_{x}+f(u), \quad x \in(0,1), t>0 \\
v_{t}=\left(b(x) v_{x}\right)_{x}+g(v), \quad x \in(0,1), t>0 \\
z_{t}=\left(c(x) z_{x}\right)_{x}+h(z), \quad x \in(0,1), t>0 \\
u(0, t)=u(0, t)=z(0, t) \\
a(0) u_{x}(0, t)+b(0) v_{x}(0, t)+c(0) z_{x}(0, t)=0 \\
u(1, t)=v(1, t)=z(1, t)=0
\end{array}\right.
$$

onde $a, b, c \in C^{1}([0,1],(0,+\infty))$ e $f, g, h$ satisfazem $(H 1)$ e $(H 2)$. Não podemos reescrever tal sistema como uma equação unidimensional, daí a dificuldade em utilizarmos os argumentos de [1] e [12]. Investigar a tranversalidade das variedades estável e instável para tais problemas é um assunto importante que será tema de futura pesquisa. 


\section{Bibliografia}

[1] S.B. Angenent, The Morse-Smale Property for a Semi-linear Parabolic Equations, Journal of Differential Equations 62 (1986), 427-442.

[2] S. Agmon, Unicité et Convexité dans les Problèmes Differentielles, Univ. of Montreal Press, Montreal (1986).

[3] G. Caginalp And W. Xie, Mathematical Analysis of Phase Memory Alloys, Pitman Research Notes in Mathematics Series 363 (1996), 61-88.

[4] V. Carbone, Problemas Parabólicos em Materiais Compostos Unidimensionais. Propriedade de Morse-Smale, Tese de doutorado, ICMC-USP (2003).

[5] A.N. Carvalho And S. Piskarev, A General Approximation Scheme for Attractors of Abstract Parabolic Problems, preprint.

[6] M.G. Crandall and P.H. Rabinowitz, Bifurcation from Simple Eigenvalues, J. Functional Analysis 8 (1971), 321-340.

[7] M.G. Crandall and P.H. Rabinowitz, Bifurcation, Pertubation of Simple Eigenvalues and Linearized Stability, Arch. Rat. Mech. Anal. 52 (1973), 161-181.

[8] J.K. Hale, Asymptotic Behavior of Dissipative Systems, Mathematical Surveys and Monographys 25, AMS (1988).

[9] J.K. Hale And Massat, Asymptotic Behavior of Gradient-like Systems, Dynamical Systems II, Academic Press (1982), 85-101.

[10] J.K. Hale and G. Raugel, A Reaction-Diffusion Equation on a Thin L-Shaped Domain, Proc. Roy. Soc. Edinburgh Sect. A 125 (1995), 283-327. 
[11] D. HenRy, Geometric Theory of Semilinear Parabolic Equations, Lectures Notes in Mathematics 840, Springer-Verlag (1981).

[12] D. Henry, Some Infinite-Dimensional Morse-Smale Systems Defined by Parabolic Partial Differential Equations, Journal of Differential Equations 59 (1985), 165-205.

[13] E.L. INCE, Ordinary Differential Equation, Dover Publications (1956).

[14] H. Matano, Non Increase of the Lapnumber of a Solution for a One Dimensional Semilinear Parabolic Equation, J. Fac. Sci. Univ. Tokyo IA Math. 29 No. 2 (1982), 401-441.

[15] A. PAZY, Semigroups of Linear Operadors and Applications to Partial Differential Equations, Springer-Verlag (19.).

[16] M.H. Protter And H.F. Weinberger, Maximum Principles in Differential Equations, Prentice-Hall, New York (1956).

[17] P.H. Rabinowitz, Some Global Results for Nonlinear Eigenvalue Problems, J. Funct. Anal. 7 (1971), 487-513.

[18] Rocha, Bifurcation Diagrams for a Parabolic Equation, Tese de Doutorado .

[19] J.A. Smoller, Shock -Waves and Reaction-Diffusion Equations, Grundlehren Math. Wiss. 258, Springer-Verlag (1983).

[20] R. TEMAM, Infinite Dimensional Dynamical Systems in Mechanics and Physics, Springer-Verlag (1980). 